\title{
LA-13519-T
}

RECEIVED

FEB 161999

Crenulative Turbulence in a

\section{OS II}

Converging Nonhomogeneous Material

\section{Los Alamos \\ NATIONAL LABORATORY}

Los Alamos National Laboratory is operated by the University of California for the United States Department of Energy under contract W-7405-ENG-36. 
This thesis was accepted by the Department of Mechanical Engineering, University of New Mexico, Albuquerque, New Mexico, in partial fulfilment of the requirements for the degree of Master of Science. The text and illustrations are the independent work of the author and only the front matter has been edited by the CIC-1 Writing and Editing Staff to conform with Department of Energy and Los Alamos National Laboratory publication policies.

An Affirmative Action/Equal Opportunity Employer

This report was prepared as an account of work sponsored by an agency of the United States Government. Neither The Regents of the University of California, the United States Government nor any agency thereof, nor any of their employees, makes any warranty, express or implied, or assumes any legal liability or responsibility for the accuracy, completeness, or usefulness of any information, apparatus, product, or process disclosed, or represents that its use would not infringe privately owned rights. Reference herein to any specific commercial product, process, or service by trade name, trademark, manufacturer, or otherwise, does not necessarily constitute or imply its endorsement, recommendation, or favoring by The Regents of the University of California, the United States Government, or any agency thereof. The views and opinions of authors expressed herein do not necessarily state or reflect those of The Regents of the University of California, the United States Government, or any agency thereof. Los Alamos National Laboratory strongly supports academic freedom and a researcher's right to publish; as an institution, however, the Laboratory does not endorse the viewpoint of a publication or guarantee its technical correctness. 


\section{DISCLAIMER}

Portions of this document may be illegible in electronic image products. Images are produced from the best available original document. 
Crenulative Turbulence in a

Converging Nonhomogeneous Material

Casildo A. Romero

\section{Los Alamos}

NATIONAL LABORATORY

Los Alamos, New Mexico 87545 
6.5 Individual Components of the $Z_{i j}$ Transport Equation . . . . 73

6.6 Mean Internal Energy, Mean Kinetic Energy, and the Turbulent Kinetic Energy . . . . . . . . . . . . . . . 74

6.7 Summary of the Spherical Formulation . . . . . . . . . 75

7 Properties of the Modeled Governing Equations 76

7.1 Primary Driver for Crenulative Turbulence . . . . . . . 76

7.2 The Limit as the Nonhomogeneities Vanish . . . . . . . . . . 77

7.3 A First Order Approximation for Behavior of $Z_{i j} \ldots \ldots \ldots 79$

7.4 Cosine Distribution of the Instantaneous Kinematic Viscosity . . 83

7.5 Discontinuous Distribution of the Instantaneous

7.6 Positive Definite Entropy Changes . . . . . . . . . . . . . 91

7.7 Summary of the Properties . . . . . . . . . . . 92

8 Nondimensional Governing Equations, Nondimensional Initial and Boundary Conditions 94

8.1 Nondimensional Governing Equations . . . . . . . . . . . 95

8.2 Nondimensional Initial and Boundary Conditions . . . . . . . 99

$9 \quad$ Results and Conclusions 102

$9.1 \quad$ Homogeneous Case . . . . . . . . . . . . . . . . . 103

9.2 Base Case . . . . . . . . . . . . . . . . . 105

9.3 Variations of Reynolds Number . . . . . . . . . . . 110

9.4 Variation of $\beta \ldots \ldots \ldots \ldots \ldots \ldots \ldots \ldots$

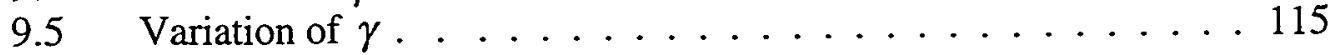

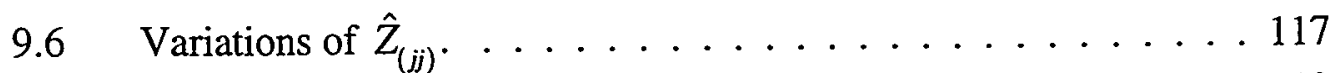

9.7 Enhancement to the Return to Isotropy . . . . . . . . . . . 119

9.8 Summary and Conclusions . . . . . . . . . . . . . 121

Appendix A Numerics 124

A.1 Numerical Scheme . . . . . . . . . . . . . . . 124

A.2 Boundary Conditions in Finite Difference Form. . . . . . . . 127

A.3 Convergence Test for Homogeneous Case. . . . . . . . . 128

$\begin{array}{ll}\text { References } & 129\end{array}$ 


\section{List of Figures}

1.1 Rayleigh-Taylor Instability 1

1.2 Nova Experiment at Lawrence Livermore Laboratory 3

1.3 Macroscopic DNS Calculation 4

1.4 Meso-Scale DNS Calculation 5

1.5 Large Scale DNS Calculation 6

3.1 Instantaneous Velocity in Terms of Mean and Fluctuating Components 17

3.2 Ensemble Average of the Instantaneous Velocity 18

5.1 Stretching of a Nonhomogeneity 59

6.1 Spherical Coordinate System 66

7.1 First Order Approximation for $Z_{i j} \quad 81$

7.2 Elongation of the Nonhomogeneities During Spherical Convergence 82

7.3 Discontinuous Distribution of Kinematic Viscosity in Radial Direction 88

7.4 Unit Cell of the Nonuniform Distribution 89

9.1 Comparison of Homogeneous Case to Nonhomogeneous Case A 104

9.2 Comparison of Homogeneous Case to Nonhomogeneous Base Case 106

9.3 Different Energies for the Nonhomogeneous Base Case 107

$9.4 \hat{M}_{i j}$ Components for the Base Case 108

$\begin{array}{lll}9.5 & \hat{Z}_{i j} \text { Components for the Base Case } & 109\end{array}$

9.6 Comparison of Homogeneous Case B to Nonhomogeneous Case B 111

9.7 Comparison of Homogeneous Case $\mathrm{C}$ to Nonhomogeneous Case $\mathrm{C} \quad 112$

9.8 Turbulence Energy for the Base Case, Case B, and Case C 113

9.9 Comparison of Internal Energy for Nonhomogeneous Base Case and Nonhomogeneous Case D 114

9.10 Comparison of Turbulence Energy for Nonhomogeneous Base Case and Nonhomogeneous Case D 
9.11 Comparison of Internal Energy for Nonhomogeneous Base Case and Nonhomogeneous Case E

9.12 Comparison of Turbulence Energy for Nonhomogeneous Base Case and Nonhomogeneous Case E

9.13 Comparison of Internal Energy for Nonhomogeneous Base Case, Nonhomogeneous Case F, and Nonhomogeneous Case G

9.14 Comparison of Turbulence Energy for Nonhomogeneous Base Case, Nonhomogeneous Case F, and Nonhomogeneous Case G

9.15 Comparison of $\hat{Z}_{i j}$ Component Distribution for the Nonhomogeneous Base Case and Nonhomogeneous Case $\mathrm{H}$

A.1 Finite Difference Grid 126

A.2 Finite Difference Domain with Imaginary Control Volumes 127

A.3 Convergence Test for Homogeneous Case 128 


\section{List of Symbols}

$R_{i j} \quad$ Reynolds Stress Tensor

$\hat{R}_{i j} \quad$ Nondimensional Reynolds Stress Tensor

$M_{i j} \quad$ First Order Response Tensor

$\hat{M}_{i j} \quad$ Nondimensional First Order Response Tensor

$Z_{i j} \quad$ Driving Tensor

$\hat{Z}_{i j} \quad$ Nondimensional Driving Tensor

$\hat{Z}_{(j)} \quad$ Diagonal Components of the Nondimensional Driving Tensor

$Z_{\text {trace }} \quad$ Trace of the Driving Tensor $Z_{i j}$ for Vector Notation

$N_{i} \quad$ Turbulence Advection of the Kinematic Viscosity Coefficient

$e \quad$ Instantaneous Internal Energy

$\vec{e} \quad$ Mean Internal Energy

$\hat{\bar{e}} \quad$ Nondimensional Mean Internal Energy

K Turbulent Kinetic Energy

KE Mean Kinetic Energy

$C_{D} \quad$ Constant Modeling Parameter for the Turbulence Self Diffusion in $R_{i j}$

Equation

$C_{B} \quad$ Constant Modeling Parameter for the Rapid Pressure in $R_{i j}$ Equation

$C_{R} \quad$ Constant Modeling Parameter for the Return to Isotropy in $R_{i j}$ Equation

$C_{H R} \quad$ Constant Modeling Parameter for the Collision of Nonhomogeneities in $R_{i j}$ Equation

$C_{H} \quad$ Constant Modeling Parameter for the Turbulence Diffusion in $M_{i j}$

Equation

$C_{M} \quad$ Constant Modeling Parameter for the Return to Isotropy in $M_{i j}$ Equation

$C_{z} \quad$ Constant Modeling Parameter for the Return to Isotropy in $M_{i j}$ Equation 
$C_{X} \quad$ Constant Modeling Parameter for the Turbulence Diffusion in $Z_{i j}$ Equation

$\rho \quad$ Instantaneous Mass Density

$u_{i} \quad$ Instantaneous Velocity Vector

$\bar{u}_{i} \quad$ Mean Velocity Vector

$u_{i}^{\prime} \quad$ Fluctuating Velocity Vector

$u_{r} \quad$ Instantaneous Radial Velocity in Spherical Coordinates

$\bar{u}_{r} \quad$ Mean Radial Velocity in Spherical Coordinates

$\bar{u}_{\text {reference }} \quad$ Reference Mean Velocity at the Outer Radius in Spherical Coordinates

$\sigma_{i j} \quad$ Total Stress Tensor

$\tau_{i j} \quad$ Shear Stress

E Instantaneous Total Energy

$q_{j} \quad$ Conductive Heat Flux

$P \quad$ Thermodynamic Pressure

$p \quad$ Ratio of the Thermodynamic Pressure to the Instantaneous Mass Density

$\bar{p} \quad$ Mean Component of $p$

$p^{\prime} \quad$ Fluctuating Component of $p$

$\delta_{i j} \quad$ Kronecker Delta

$\lambda \quad$ Bulk Viscosity Coefficient

$\mu \quad$ Dynamic Viscosity Coefficient

$v \quad$ Kinematic Viscosity Coefficient

$\bar{v} \quad$ Mean Kinematic Viscosity Coefficient

$v^{\prime} \quad$ Fluctuating Kinematic Viscosity Coefficient

$k \quad$ Thermal Conductivity

$T \quad$ Temperature

$S(\rho, T, \ldots) \quad$ Source to Evolution Equation of Dynamic Viscosity Coefficient 
$s_{x} \quad$ Separation Scale Between Nonhomogeneities in the $x$ Direction

$s_{y} \quad$ Separation Scale Between Nonhomogeneities in the $y$ Direction

$s_{z} \quad$ Separation Scale Between Nonhomogeneities in the $z$ Direction

$s_{r} \quad$ Separation Scale Between Nonhomogeneities in the $r$ Direction

$s_{\theta} \quad$ Separation Scale Between Nonhomogeneities in the $\theta$ Direction

$s_{\phi} \quad$ Separation Scale Between Nonhomogeneities in the $\phi$ Direction

$s_{o} \quad$ Initial Isotropic Separation Scale Between Nonhomogeneities

$\varepsilon_{i j} \quad$ Dissipation in a $K-\varepsilon$ Turbulence Model

$\Delta \quad$ Function of Turbulent Reynolds Number (Modeling Parameter)

$Q \quad$ Enhancement to the Decay in $R_{i j}$ (Modeling Parameter)

$w_{1} \quad$ Nondimensional Enhancement to the Decay in $R_{i j}$ (Modeling Parameter)

$\tilde{Q} \quad$ Enhancement to the Decay in $M_{i j}$ (Modeling Parameter)

$w_{2} \quad$ Nondimensional Enhancement to the Decay in $M_{i j}$ (Modeling Parameter)

$D_{i j} \quad$ Modeling Tensor in $Z_{i j}$ for Triple Correlations

$B(t) \quad$ Incompressibility Parameter for Spherical Symmetric Convergence

B Constant Incompressibility Parameter for Spherical Symmetric

Convergence

$\xi_{i j} \quad$ Perturbation from Initial State of $Z_{i j}$

$R_{2} \quad$ Outer Radius of the Spherical Shell of Fluid

$R_{i} \quad$ Inner Radius of the Skin with Variable Viscosity of the Nonhomogeneity

$R_{o} \quad$ Outer Radius of the Skin with Variable Viscosity of the Nonhomogeneity

$\bar{R} \quad$ Nominal Radius of the Skin with Variable Viscosity of the

Nonhomogeneity

$a$

Skin Thickness with Variable Viscosity of the Nonhomogeneity

$\delta v \quad$ Difference Between the Maximum and Minimum Values of the

Instantaneous Kinematic Viscosity

$f_{x} \quad$ Elongation Factor of the Nonhomogeneities in the $x$ Direction 
$f_{y} \quad$ Elongation Factor of the Nonhomogeneities in the $y$ Direction

$f_{z} \quad$ Elongation Factor of the Nonhomogeneities in the $z$ Direction

Re Reynolds Number (Nondimensional Parameter)

$\beta \quad$ Measure of the Scale of the Nonhomogeneity relative to the Size of the

Spherical Shell (Nondimensional Parameter)

$\gamma \quad$ Measure of the Level of Nonhomogeneity in the Spherical Shell

(Nondimensional Parameter)

$\eta \quad$ Convergence Parameter in the Spherical Coordinate System

(Nondimensional Parameter) 


\title{
Crenulative Turbulence in a Converging Nonhomogeneous Material
}

by

Casildo A. Romero

\begin{abstract}
Crenulative turbulence is a nonlinear extension of the Bell-Plesset instability, usually observed in a converging system in which there is a nonhomogeneous response of stress to strain and/or strain rate. In general, crenulation occurs in any circumstance in which the mean flow streamlines converge the material more strongly than the compressibility can accommodate. Elements of the material slip past each other, resulting in local fluctuations in velocity from that of the mean flow, producing a type of turbulence that is more kinematic than inertial. For a homogeneous material, crenulation occurs at the atomic or molecular scale. With nonhomogeneous stress response at larger scales, the crenulative process can also occur at those larger scales. The results are manifested by a decrease in the rate of dissipation to heat, and by the configurationallyirreversible mixing of nonhomogeneities across any mean-flow-transported interface.

We obtain a mathematical description of the crenulative process by means of Reynolds decomposition of the appropriate variables, and the derivation of transport equations for the second-order moments that arise in the mean-flow momentum and energy equations. The theory is illustrated by application to the spherical convergence of an incompressible fluid with nonhomogeneous distribution of kinematic viscosity.
\end{abstract}




\section{Chapter 1}

\section{Introduction}

Fluid and material instabilities have been of interest to scientists and engineers for decades. Predicting the behavior of these instabilities has relied on mathematical modeling of the physical phenomenon associated with the instability. In recent years the ability to model these instabilities has improved greatly with the help of high-speed computers and increased knowledge of modeling the ensemble-averaged Navier Stokes equations. There are various fluid and material instabilities, but the Rayleigh-Taylor, Kelvin-Helmholtz, and Richtmyer-Meshkov instabilities are probably the three most studied, but in recent years the Bell-Plesset instability has gained some attention.

In the growth of these instabilities a perturbation and some physical driving mechanism are needed to initiate the growth of the instability. In the case of the RayleighTaylor instability a low amplitude perturbation at the planar interface between a high density fluid and a low density fluid, and a pressure gradient normal to the planar interface will sustain the growth of the instability as shown in Fig. 1.1 [1]. In Fig. 1.1 the high density is on the top of the low density.
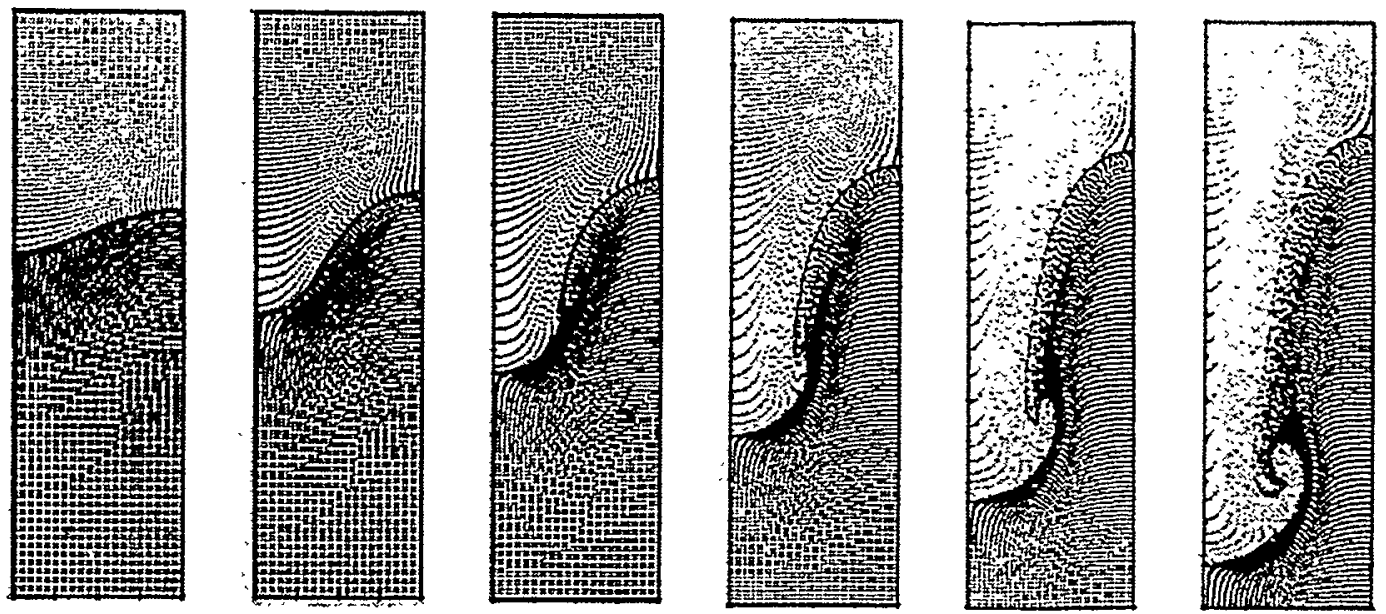

Figure 1.1 Rayleigh-Taylor Instability 
Once the instability begins to grow it will first enlarge without mode coupling then transform to a configuration of spikes and bubbles, next it will couple to more complicated structures (mushrooms), and finally go to a completely turbulent state with strong coupling to both small and large scales. In the case of the Kelvin-Helmholtz instability a low amplitude perturbation at a slip interface within a fluid or between two different fluids, and a mean velocity shearing across the interface will sustain the growth of instability. Once the instability begins to grow it will develop both large scale roles and fine scale turbulence [2]. In the case of Bell-Plesset instability a nonhomogeneity couples to contortions in mean velocity variations, for example during convergence, to sustain the growth of the instability $[3,4]$. The growth of the instability occurs in the bulk of the material; in convergence it is attributed to the development of hoop stresses in the material. There are situations where both a Rayleigh-Taylor component and a Bell-Plesset component are present, and they can sometimes be difficult to differentiate $[5,6]$.

The goal for this thesis is to describe the theoretical foundations of the nonlinear extension of the Bell-Plesset instability, which is called "crenulation turbulence". The particular set of circumstances we have chosen to display crenulation turbulence is in a spherical converging geometry. The nonhomogeneity we have chosen by which to illustrate the process is a variation in material stress response arising from the fluctuations in kinematic viscosity of a Newtonian fluid. Ultimately our goal is to characterize crenulation in an elastic material (e.g. a metal) with variations in strength of yield to plastic deformation, which occurs on the scale of grains and exhibits much influence from the texture of the material. P.V. Makarov has investigated plastic flow, prefailures, and subsequent failure of all materials and has concluded it is related to structural inhomogeneities of different scales [7]. At this stage, we have chosen a Newtonian fluid with continuous variations in kinematic viscosity as the simplest material for which there is an exact set of microscopic equations that are everywhere continuous to serve as a basis for developing the theory, thus providing a foundation for metals with fine scale discontinuous 
variations in yield strength. At this stage of development we can see the consequences for growth of the instability in several important manifestations, for example in its effects on heat dissipation.

It has been observed that crenulation preserves some memory of its initial conditions at the highly nonlinear stages, particularly in the near absence of coupling to longer wavelength modes. In the Nova experiments conducted at the Lawrence Livermore National Laboratory the perturbation was initially placed on the outer surface of a cylinder and eventually fed through to the bulk of the material and to the inner surface [6]. Due to the initial pressure gradient of the laser-beam driver a Rayleigh-Taylor component existed, but once the pressure gradient vanished the system was in a state of "coasting convergence" and the Rayleigh-Taylor component subsided. During this state of coasting convergence the crenulative component was dramatically manifested, as shown in Fig. 1.2.
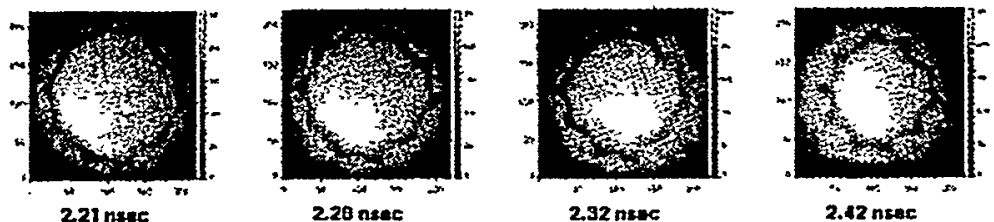

220 nsec

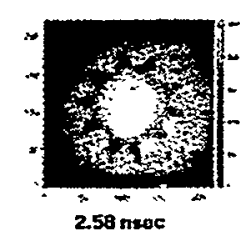

2.42 nese
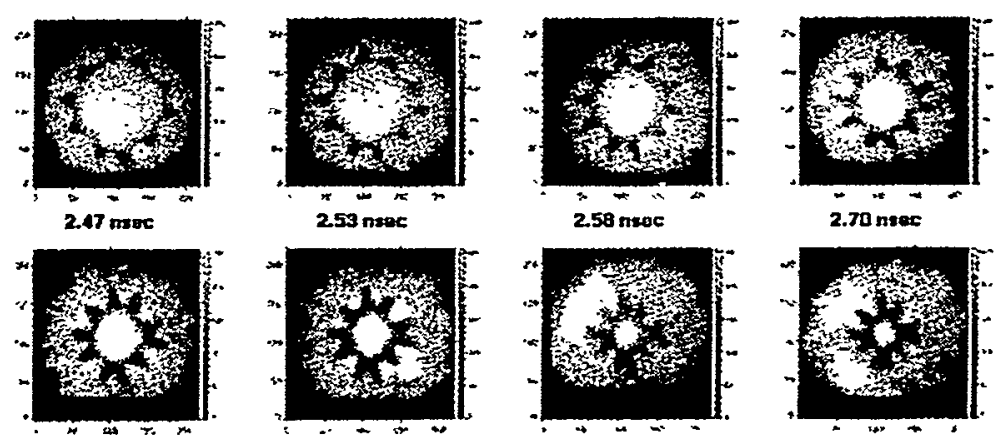

2.81 neat s.00 noec

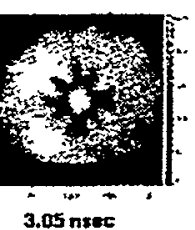

Figure 1.2 Nova Experiment at Lawrence Livermore Laboratory

Crenulation is an instability that can occur at a macroscopic or microscopic level. Figure 1.2 shows long wavelength modes as the dominant structural scales. At the macroscopic level the hoop stresses force bulk regions of material, associated with some macroscopic perturbation or nonhomogeneity, to move relative to other regions of material. 
At the other extreme, the perturbations are at the molecular or atomic scale. Combinations of these extremes can be seen in a direct numerical simulation (DNS) by Brad Holian and Peter Lomdahl at the Los Alamos National Laboratory, as shown in Fig. 1.3 [8]. In this calculation a three-dimensional cylinder was composed of 400,000 atoms and the characteristic energy of interactions between the atoms followed a Lennard-Jones (6-12) potential. The initial configuration of the particles was as though the cylinder was cut from a single crystal, so as to have a preferential direction. The preferential direction introduced a nonisotropy at large scale into the system, which dominated in the growth of convergence-crenulation amplitude
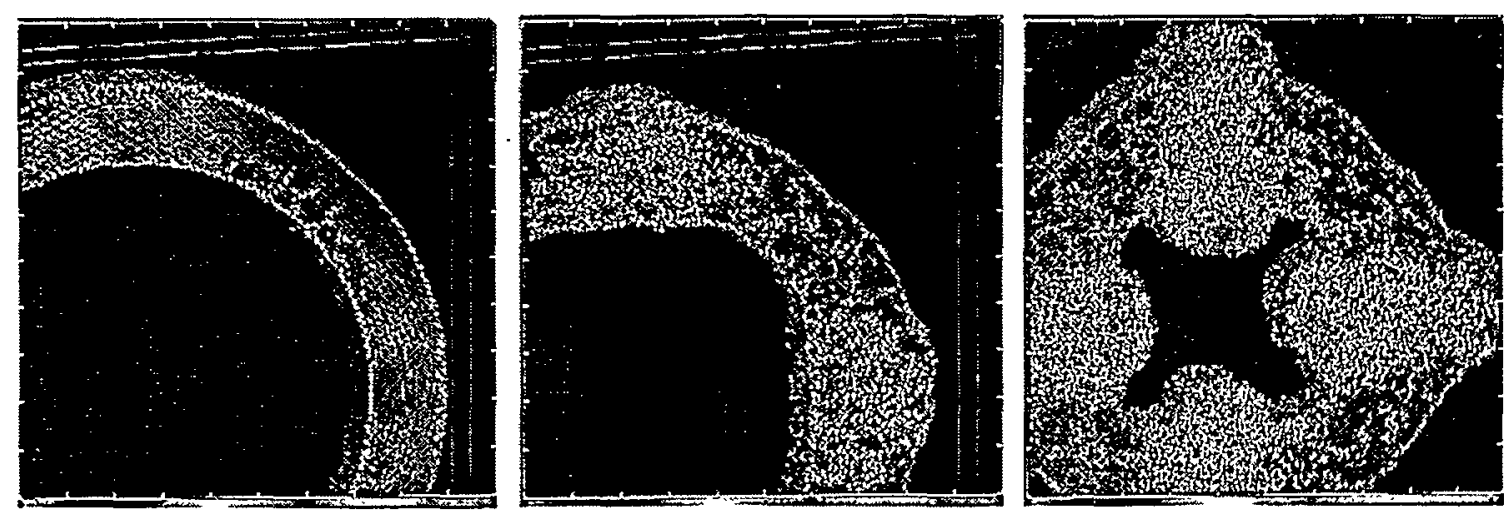

Figure 1.3 Macroscopic DNS Calculation

A second calculation by Lomdahl and Holian had an initial nonhomogeneous atomic arrangement in meso-scale crystals, and showed crenulation occurring at an intermediate size scale, as shown in Fig. 1.4 

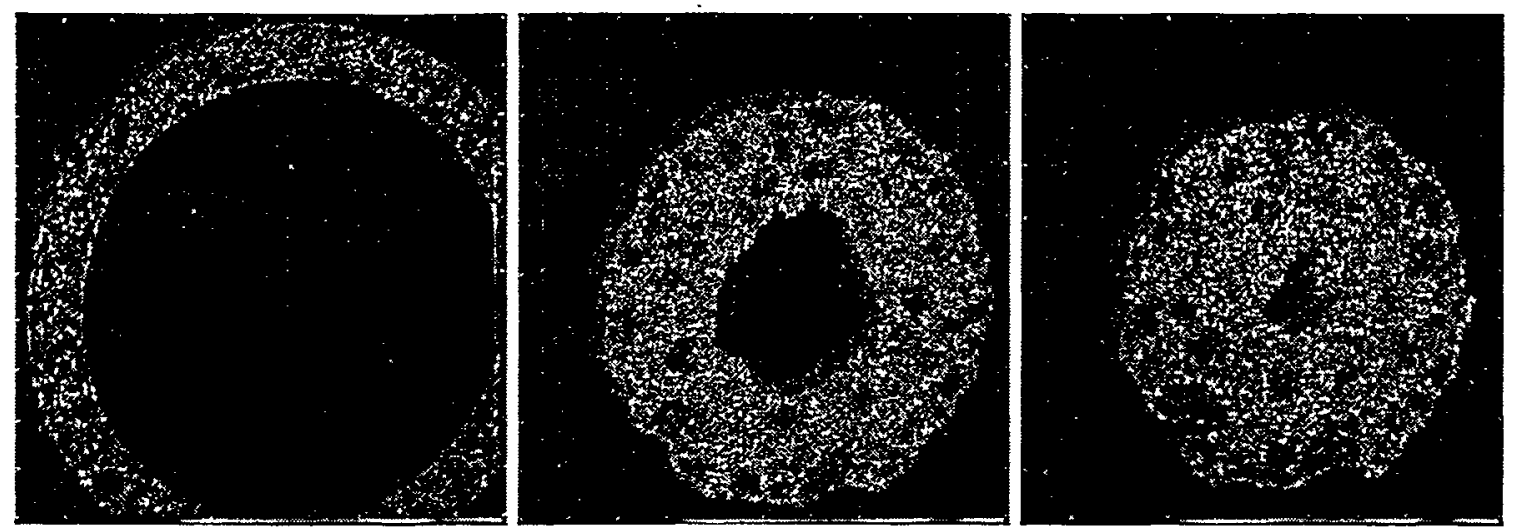

Figure 1.4 Meso-Scale DNS Calculation

If the particles are completely random in spacing the behavior is that of crenulation at an atomic level, which can be described by homogeneous viscous or plastic behavior.

The phenomenon seen in the meso-scale DNS can also be seen in the large scale direct numerical simulation done by Bruce Trent [9], as shown in Fig. 1.5. 

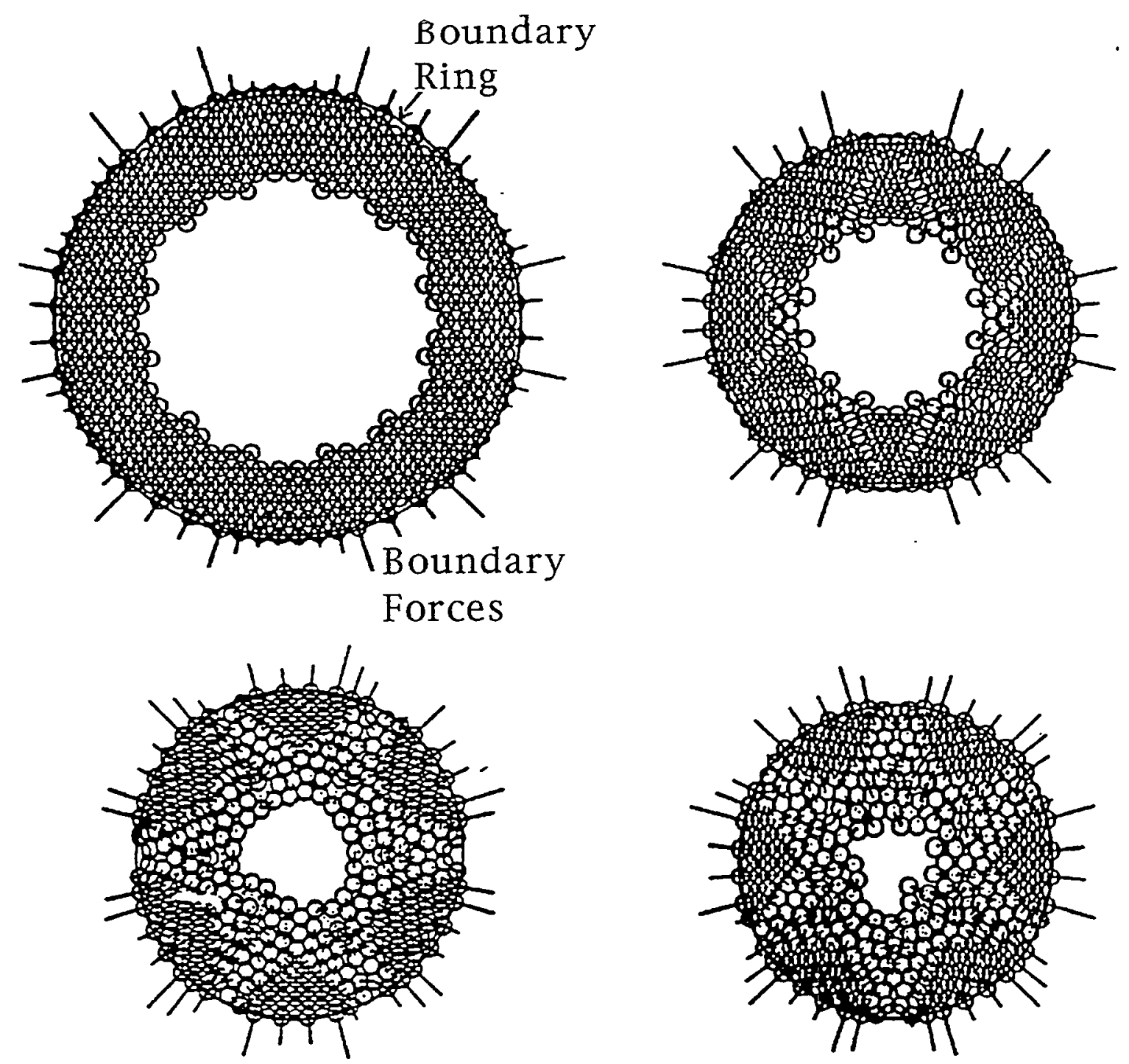

Figure 1.5 Large Scale DNS Calculation

In the microscopic case the hoop stresses force molecules of the material, associated with microscopic perturbations of their placement, to move relative to other molecules. The crenulation occurring at the microscopic level is described by the limit as the collective nonhomogeneities vanish.

The description of crenulative turbulence is assumed to be completely contained in the Navier-Stokes equations for fluid dynamics. Just as for ordinary turbulence, however, the essence of the behavior is difficult to extract from these equations. More tractable is to investigate several types of averages, and to derive transport equations for quantities that 
emerge from the averaging process as a result of nonlinear fluid behavior. The technique seems at first to magnify the complexity enormously, but much progress has been made with this approach, which is the one that we take in this thesis. A basic feature of this way of proceeding is the recognition of mean quantities and the fluctuations of those quantities from the mean values. Thus to characterize the crenulative behavior we have taken a Reynolds decomposition approach, much like that of current turbulence transport theories [10]. The continuity of the instantaneous fluid variables in the Navier-Stokes equations has facilitated our taking this approach. Assuming the density is constant we make a Reynolds decomposition of the fluid variables; velocity, pressure, and kinematic viscosity. Various turbulence variables emerge from the derivation of the transport equations. The most important variables are the Reynolds stress tensor $R_{i j}$, the response tensor $M_{i j}$, the $N_{i}$ vector, and the driving tensor $Z_{i j}$. The Reynolds stress tensor for crenulation is the same as the Reynolds stress tensor for classical turbulence and is a measure of the turbulence intensity. The response tensor $M_{i j}$, when coupled to the mean flow rate-of-strain, is a source to the Reynolds stress tensor.

$$
\frac{\partial R_{i j}}{\partial t}=M_{j l}\left(\frac{\partial \bar{u}_{i}}{\partial x_{l}}+\frac{\partial \bar{u}_{l}}{\partial x_{i}}\right)+M_{i l}\left(\frac{\partial \bar{u}_{j}}{\partial x_{l}}+\frac{\partial \bar{u}_{l}}{\partial x_{j}}\right)+\ldots
$$

The driving tensor $Z_{i j}$ describes the configuration of the nonhomogeneities in the system. Also, when the driving tensor is coupled to the rate of strain it is a source to the response tensor $M_{i j}$ and sets the scale for the evolving crenulation.

$$
\frac{\partial M_{i j}}{\partial t}=Z_{j k}\left(\frac{\partial \bar{u}_{i}}{\partial x_{k}}+\frac{\partial \bar{u}_{k}}{\partial x_{i}}\right)+\ldots
$$

It can be seen the driving tensor coupled to the mean flow gradient ultimately contributes to the Reynolds stress tensor. Physically this means the crenulative turbulence is driven by 
the combination of a nonhomogeneity and the convergence of the material. These terms are very important because they fall directly out of the derivation; there is no modeling involved and foundation of the physics lies in these terms. Our goal in this thesis is to see in detail how these and all the other relevant transport contributions emerge from the derivations. 


\section{Chapter 2}

\section{General Equations}

In this chapter the basic equations for conservation, for the evolution of the kinematic viscosity, and for the constitutive responses are introduced and discussed in some detail. These equations are expressed in an Eulerian frame of reference and in tensor notation. Also in this chapter the dependent variables, the instantaneous fluid variables, are introduced. These fluid variables include the mass density, velocity, pressure, dynamic viscosity, kinematic viscosity, internal energy, and temperature. The equations in this chapter are for the exact microscopic variables, not the mean variables described in Chapter 3.

In the absence of body sources, any quantity per unit volume, $A$, changes with time in response to a flux of that quantity, $F_{j}$, according to the equation

$$
\frac{\partial A}{\partial t}+\frac{\partial F_{j}}{\partial x_{j}}=0
$$

In this conservation equation, $A$ can be a tensor of any order ( scalar, vector, etc.) and $F_{j}$ is accordingly a next-order tensor.

\subsection{Conservation of Mass}

Consider a control volume with mass that changes with time as a result of flux across the boundary. For the principle of conservation of mass, the total flux is purely advective, and is given by $F_{j}=\rho u_{j}$. Thus the mass conservation is represented mathematically by 


$$
\frac{\partial \rho}{\partial t}+\frac{\partial\left(\rho u_{j}\right)}{\partial x_{j}}=0
$$

where $\rho$ is the instantaneous mass density and $u_{j}$ is the instantaneous fluid velocity. If the mass density is assumed to be constant through space and time we get the incompressibility condition which is represented mathematically by the vanishing divergence of the velocity field (i.e. the velocity field is solenoidal)

$$
\frac{\partial u_{j}}{\partial x_{j}}=0
$$

Whether in the form of equation (2.1) or equation (2.2) the conservation of mass is the first condition that must be satisfied by the velocity and the mass density.

\subsection{Conservation of Momentum}

The principle of conservation of momentum is an application of Newton's second law of motion to a fluid element. The flux of momentum density, $\rho u_{i}$, has two components, the advective tensor, $\rho u_{i} u_{j}$, and the stress, $\sigma_{i j}$. Neglecting body forces the conservation of momentum can be represented mathematically by

$$
\frac{\partial\left(\rho u_{i}\right)}{\partial t}+\frac{\partial\left(\rho u_{i} u_{j}-\sigma_{i j}\right)}{\partial x_{j}}=0
$$

Under some converging circumstances gravity can play a role such as with fluid flowing unforced through a funnel, but in our case the converging forces are large compared to the gravitational effects. The fluid variables must satisfy this partial differential equation to satisfy the basic law of dynamics. The stress will be described in the section on constitutive relationships. 


\subsection{Conservation of Energy}

The principle of conservation of total energy incorporates the first law of thermodynamics for a fluid element. The conservation of total energy principle states the rate of change of the total energy is equal to the rate at which work is done plus the rate at which heat is added by conduction to the fluid element. The flux of total energy includes advection, work done, and heat conduction. The conservation of total energy can be represented mathematically by

$$
\frac{\partial(\rho E)}{\partial t}+\frac{\partial\left(\rho E u_{j}-u_{i} \sigma_{i j}+q_{j}\right)}{\partial x_{j}}=0
$$

where $E$ is the total energy per unit volume and $q_{j}$ is the heat flux due to conduction. The total energy of the fluid has two parts, the internal energy, $e$, and the total kinetic energy, $\frac{1}{2} u_{k} u_{k} ; E=e+\frac{1}{2} u_{k} u_{k}$. Using the conservation equations for mass and momentum, we can get an equation for the internal energy

$$
\frac{\partial(\rho e)}{\partial t}+\frac{\partial\left(\rho e u_{j}\right)}{\partial x_{j}}=\sigma_{i j} \frac{\partial u_{i}}{\partial x_{j}}-\frac{\partial q_{j}}{\partial x_{j}}
$$

This equation, which is equivalent to the first law of thermodynamics, describes the rate of change of the internal energy due to conversion of mechanical energy into internal energy. This is because of the action of the surface stresses, and also due to the addition of heat by

conduction. For an incompressible fluid, the second law of thermodynamics states that

$$
\frac{\partial e}{\partial t}+\frac{\partial\left(e u_{j}\right)}{\partial x_{j}} \geq 0
$$




\subsection{Constitutive Equations}

In this section an expression for the stress $\sigma_{i j}$, which occurs in the conservation of momentum and internal energy equations, and an expression for the heat flux $q_{j}$ are introduced. For the analysis of crenulative turbulence we used a Newtonian fluid constitutive relationship

$$
\sigma_{i j}=-P \delta_{i j}+\tau_{i j}
$$

where $P$ is the thermodynamic pressure, $\delta_{i j}$ is the Kronecker delta, and $\tau_{i j}$ is the shear stress, which is a function of the strain rate. An essential feature of a Newtonian fluid is that the stress is linearly related to the strain rate. The stress relationship is mathematically represented by

$$
\tau_{i j}=\lambda \delta_{i j} \frac{\partial u_{l}}{\partial x_{l}}+\mu\left(\frac{\partial u_{i}}{\partial x_{j}}+\frac{\partial u_{j}}{\partial x_{i}}\right)
$$

The coefficients $\lambda$ and $\mu$ are known as the bulk viscosity coefficient and the dynamic viscosity coefficient, respectively. If a flow is incompressible $\lambda$ has no effect on the stress since $\frac{\partial u_{l}}{\partial x_{l}}=0$. The full constitutive relationship that we use is thus

$$
\sigma_{i j}=-P \delta_{i j}+\mu\left(\frac{\partial u_{i}}{\partial x_{j}}+\frac{\partial u_{j}}{\partial x_{i}}\right)
$$

The constitutive relationship for the heat flux is an expression of Fourier's law, which states that the heat flux by conduction is proportional to the negative temperature gradient:

$$
q_{k}=-k \frac{\partial T}{\partial x_{k}}
$$

in which $k$ is the thermal conductivity of the fluid and $T$ is the temperature. 


\subsection{Evolution of the Dynamic Viscosity}

An evolution equation for the dynamic viscosity coefficient is an important facet in the analysis of crenulation. The evolution equation for the dynamic viscosity describes the change in dynamic viscosity for a given fluid element due to a source that could depend on mass density, temperature, and other fluid variables. The evolution of the dynamic viscosity is mathematically represented by

$$
\frac{D \mu}{D t}=\frac{\partial \mu}{\partial t}+u_{l} \frac{\partial \mu}{\partial x_{l}}=S(\rho, T, \ldots)
$$

where $S(\rho, T, \ldots)$ is the source. In the crenulative flow in this thesis, the dynamic viscosity is constant in any element of fluid, but varies from one element to another, thereby giving the necessary nonhomogeneity for driving the crenulation.

\subsection{Preliminary Equations for Crenulative Turbulence}

In this section we make a few assumptions to simplify the equations for our crenulative turbulence analysis. To illustrate the mathematics of crenulation it is simpler and sufficient to consider an incompressible fluid. We assume the mass density is constant through space and time so that the conservation of mass equation becomes the incompressibility condition

$$
\frac{\partial u_{j}}{\partial x_{j}}=0
$$

as stated in equation (2.2). Because the fluid is incompressible the constitutive relationship for stress simplifies to

$$
\sigma_{i j}=-P \delta_{i j}+\mu\left(\frac{\partial u_{i}}{\partial x_{j}}+\frac{\partial u_{j}}{\partial x_{i}}\right)
$$


and the bulk viscosity coefficient has no effect on the stress. We also neglect the effects of body forces acting on the fluid. The momentum equation is then divided by the constant mass density, and the simplified constitutive relationship for stress is substituted in to give

$$
\frac{\partial u_{i}}{\partial t}+\frac{\partial\left(u_{i} u_{j}\right)}{\partial x_{j}}=\frac{\partial}{\partial x_{j}}\left[-\frac{P}{\rho} \delta_{i j}+\frac{\mu}{\rho}\left(\frac{\partial u_{i}}{\partial x_{j}}+\frac{\partial u_{j}}{\partial x_{i}}\right)\right]
$$

We set $p=\frac{P}{\rho}$ and $v=\frac{\mu}{\rho}$ in which $v$ is the kinematic viscosity coefficient. Inserting $p$ and $v$ the conservation of momentum for our crenulative turbulence analysis is

$$
\frac{\partial u_{i}}{\partial t}+\frac{\partial\left(u_{i} u_{j}\right)}{\partial x_{j}}=-\frac{\partial p}{\partial x_{i}}+\frac{\partial}{\partial x_{j}}\left[v\left(\frac{\partial u_{i}}{\partial x_{j}}+\frac{\partial u_{j}}{\partial x_{i}}\right)\right]
$$

Equation (2.11), which is the combination of the conservation of momentum with the constitutive relationship for a Newtonian fluid, is a principal condition to be satisfied by the crenulating fluid. Regarding the behavior of internal energy, we simply neglect heat conduction in the fluid. With constant mass density the internal energy equation (2.5) is divided by the mass density. Substituting the simplified constitutive relationship for stress the internal energy equation becomes

$$
\frac{\partial e}{\partial t}+\frac{\partial\left(e u_{j}\right)}{\partial x_{j}}=v\left(\frac{\partial u_{i}}{\partial x_{j}}+\frac{\partial u_{j}}{\partial x_{i}}\right) \frac{\partial u_{i}}{\partial x_{j}}
$$

The work term vanishes because $\delta_{i j} \frac{\partial u_{i}}{\partial x_{j}}$ is the incompressibility condition. The terms on the right side are called the dissipation function, which measures the rate at which mechanical energy is being converted into thermal energy. The evolution of the dynamic viscosity is simplified by assuming the source is negligible. The absence of a source 
represents the idea that the dynamic viscosity of a given fluid element does not change during crenulation. With this assumption and dividing the evolution equation for the dynamic viscosity by the constant mass density gives us an evolution equation for the kinematic viscosity

$$
\frac{\partial v}{\partial t}+u_{j} \frac{\partial v}{\partial x_{j}}=0
$$

These are the microscopic equations that govern the motion of a crenulative fluid. They are the incompressibility condition, the momentum equation, the internal energy equation, and the evolution of the kinematic viscosity, i.e. equations (2.2), (2.11), (2.12), and (2.13). They could, in principle, be solved exactly, but in practice this solution is generally not tractably obtainable. Instead we look for a technique by which to extract the essence of crenulative behavior in terms of ensemble averages of the detailed behavior, as described in the following chapters. 


\section{Chapter 3}

\section{Fundamental Concepts of Turbulence}

In this chapter the ideas of a Reynolds decomposition and an ensemble average of the instantaneous fluid variables are discussed. These concepts are crucial to interpret the meaning behind the turbulence quantities that are introduced in this chapter and subsequent chapters. After the ideas of a Reynolds decomposition and an ensemble average are established, the mathematical properties associated with the combination of the two are shown. Then it is shown where the Reynolds stress tensor and the other turbulence quantities associated with crenulation arise.

\subsection{Reynolds Decomposition}

When working with a Newtonian fluid it is assumed that the Navier-Stokes equations govern the behavior of the fluid, including the turbulence behavior. When the issue of turbulence arises two question may be asked "what exactly is turbulence?" and "how do we characterize it?". Turbulence can be defined as the behavior that lies in the rapidly varying behavior of the fluid variables: velocity, density, pressure, and dynamic viscosity. The first step in characterizing turbulence was developed by Sir Osborne Reynolds, who rewrote the Navier-Stokes equations to describe the rapidly varying behavior of the fluid by making a decomposition of the fluid variables. A Reynolds decomposition is a decomposition of the instantaneous fluid variables into mean and fluctuating components; in our case $u_{i}=\bar{u}_{i}+u_{i}^{\prime}, p=\bar{p}+p^{\prime}$, and $v=\bar{v}+v^{\prime}$ where $\bar{u}_{i}, \bar{p}$, and $\bar{v}$ are the mean components of the variables and $u_{i}^{\prime}, p^{\prime}$, and $v^{\prime}$ are the fluctuating components of the variables. Consider a fixed location in a fluid where turbulence is present, and track the instantaneous velocity $u_{i}$. As time progresses we would see the velocity fluctuate about the mean velocity $\bar{u}_{i}$ as in Fig. 3.1. 


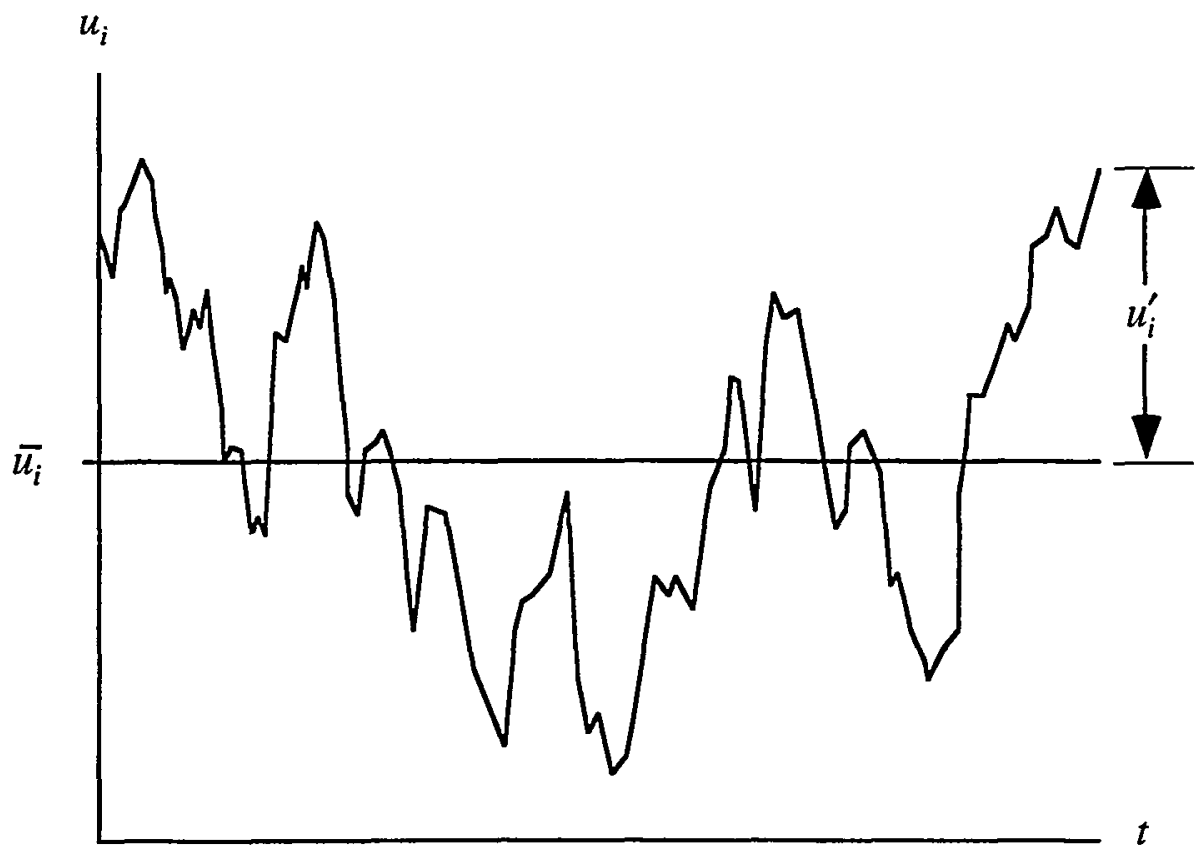

Figure 3.1: Instantaneous Velocity in Terms of Mean and Fluctuating Components

A Reynolds decomposition of the fluid variables is one of the key tools for extracting information about the turbulence behavior. The case in which the mass density and dynamic viscosity are constant throughout space and time is known as "constant density turbulence" or "classical turbulence". This type of turbulence research has been studied for decades and most of the engineering turbulence transport models being used today have stemmed from this research. The case in which the mass density is also variable through space and time is known as "variable density turbulence". This type of turbulence is fairly well known, but not as well as the constant density turbulence, having only been studied in recent years. The case in which the mass density is constant throughout space and time, but the stress response is variable is what we call "crenulative turbulence". This type of turbulence is hardly known and is the focus of this thesis. 


\subsection{Ensemble Averaging}

Ensemble averaging is a key tool in the development of turbulence transport theory. It is the idea that the governing equations can be averaged even though the rapid variations due to turbulence play a significant role. An ensemble average can be equivalent to a time average, or spatial average, but in general it is an average over many experiments with the same macroscopic initial and boundary conditions. Consider the ensemble average over many experiments of the flow over a cylinder. If we were to run this experiment one time and look at the instantaneous velocity at position $\left(x_{o}, y_{o}, z_{o}\right)$ at time $t_{o}$ the velocity might be as Figure 3.2 indicates for experiment \#1.

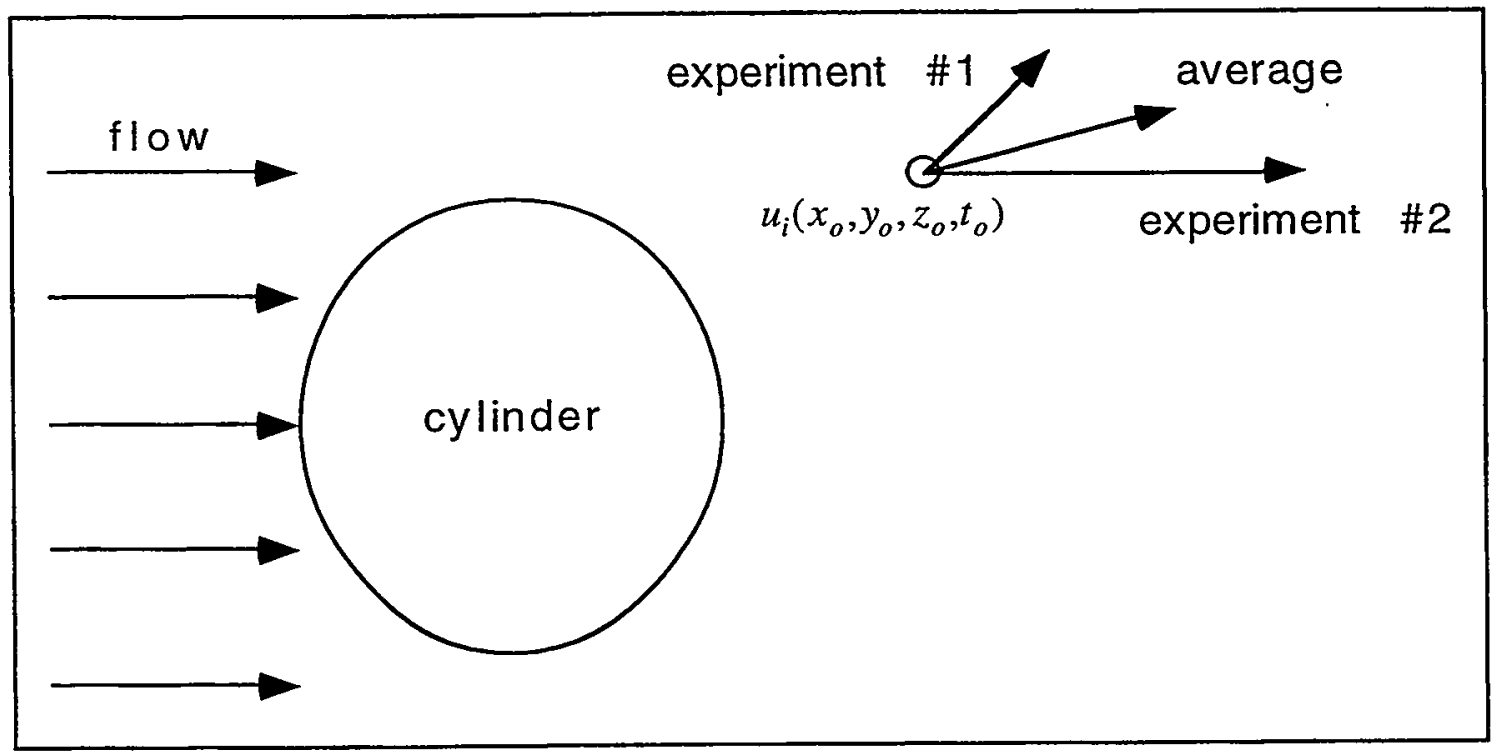

Figure 3.2: Ensemble Average of the Instantaneous Velocity

If we were to run the experiment a second time and look at the instantaneous velocity at the same position $\left(x_{o}, y_{o}, z_{o}\right)$ and at the same time $t_{o}$ as in experiment \#1, the velocity might be as the experiment \#2 label indicates. If we were to continue to run the experiment over and over we would find the velocity at position $\left(x_{o}, y_{o}, z_{o}\right)$ and at time $t_{o}$ would deviate about some average velocity $\bar{u}_{i}$. We relate these ideas to the governing equations by taking an ensemble average of the governing equations. If the initial and boundary conditions were 
exactly the same, then each member of the ensemble of solutions would be the same as every other member. Thus the ensemble of solutions becomes interesting for turbulence studies only if there are "microscale" differences among the initial and boundary conditions. We signify an ensemble average of the governing equations (2.2), (2.11), (2.12), and (2.13) by a bar over each term

$$
\begin{aligned}
& \overline{\frac{\partial u_{l}}{\partial x_{l}}}=0 \\
& \overline{\frac{\partial u_{i}}{\partial t}}+\overline{\frac{\partial u_{i} u_{l}}{\partial x_{l}}}=-\frac{\overline{\partial p}}{\partial x_{i}}+\overline{\frac{\partial}{\partial x_{l}}\left[v\left(\frac{\partial u_{i}}{\partial x_{l}}+\frac{\partial u_{l}}{\partial x_{i}}\right)\right]} \\
& \overline{\frac{\partial e}{\partial t}}+\overline{\frac{\partial e u_{l}}{\partial x_{l}}}=\overline{v\left(\frac{\partial u_{i}}{\partial x_{l}}+\frac{\partial u_{l}}{\partial x_{i}}\right) \frac{\partial u_{i}}{\partial x_{l}}} \\
& \frac{\overline{\partial v}}{\partial t}+\frac{\overline{\partial v u_{l}}}{\partial x_{l}}=0
\end{aligned}
$$

The ensemble average is inherently distributive, i.e. the average of the sum is equal to the sum of the averages $\overline{\alpha+\beta}=\bar{\alpha}+\bar{\beta}$. In addition the derivative of an average equals the average of the derivative.

\subsection{Properties of Reynolds Decomposition along with Ensemble Averaging}

The idea of mean and fluctuating components along with an ensemble average of the governing equations is the starting point for development of turbulence transport theory. In the governing equations (3.1), (3.2), (3.3), and (3.4) two cases occur: an instantaneous fluid variable stands alone or two instantaneous fluid variables are coupled together. A Reynolds decomposition along with an ensemble average of an instantaneous 
fluid variable or of the product of two correlated fluid variables, leads to two important properties: (1) The ensemble average of the fluctuation of an instantaneous fluid variable is zero. (2) The ensemble average of the product of two correlated variables leads to a second order correlation

(1) $\overline{\bar{\alpha}+\alpha^{\prime}}=\bar{\alpha}+\overline{\alpha^{\prime}}=\bar{\alpha} \Rightarrow \overline{\alpha^{\prime}}=0$

(2) $\overline{\left(\bar{\alpha}+\alpha^{\prime}\right)\left(\bar{\beta}+\beta^{\prime}\right)}=\bar{\alpha} \bar{\beta}+\bar{\alpha} \overline{\beta^{\prime}}+\overline{\alpha^{\prime} \bar{\beta}}+\overline{\alpha^{\prime} \beta^{\prime}}=\bar{\alpha} \bar{\beta}+\overline{\alpha^{\prime} \beta^{\prime}}$

The ensemble average of a Reynolds decomposition of two correlated instantaneous fluid variables can be in combination with spatial gradients or temporal gradients of any order. The second order correlation is some measure of the intensity of the fluctuations of the correlated variables. Also, the correlation can be thought of as a single variable; $A=\overline{\alpha^{\prime} \beta^{\prime}}$. Properties (1) and (2) can be extended to a Reynolds decomposition and ensemble average of three correlated variables

$$
\begin{gathered}
\overline{\left(\bar{\alpha}+\alpha^{\prime}\right)\left(\bar{\beta}+\beta^{\prime}\right)\left(\bar{\gamma}+\gamma^{\prime}\right)}=\bar{\alpha} \bar{\beta} \bar{\gamma}+\bar{\alpha} \bar{\beta} \overline{\gamma^{\prime}}+\bar{\alpha} \overline{\beta^{\prime}} \bar{\gamma}+\overline{\alpha^{\prime}} \bar{\beta} \bar{\gamma} \\
+\bar{\alpha} \overline{\beta^{\prime} \gamma^{\prime}}+\overline{\alpha^{\prime} \gamma^{\prime}} \bar{\beta}+\overline{\alpha^{\prime} \beta^{\prime}} \bar{\gamma}+\overline{\alpha^{\prime} \beta^{\prime} \gamma^{\prime}}
\end{gathered}
$$

A variety of terms appear, but the average of a single fluctuation is always zero. Therefore only the product of the three mean quantities, the second order correlations, and the third order correlation do not vanish

$$
\overline{\left(\bar{\alpha}+\alpha^{\prime}\right)\left(\bar{\beta}+\beta^{\prime}\right)\left(\bar{\gamma}+\gamma^{\prime}\right)}=\bar{\alpha} \bar{\beta} \bar{\gamma}+\overline{\alpha \beta^{\prime} \gamma^{\prime}}+\overline{\alpha^{\prime} \gamma^{\prime}} \bar{\beta}+\overline{\alpha^{\prime} \beta^{\prime}} \bar{\gamma}+\overline{\alpha^{\prime} \beta^{\prime} \gamma^{\prime}}
$$




\subsection{Reynolds Decomposition and Ensemble Average of the Mass, Momentum, Internal Energy, and Kinematic Viscosity Equations}

The mass equation (incompressibility condition), momentum, internal energy, and kinematic viscosity equations along with a Reynolds decomposition of the instantaneous fluid variables and an ensemble average results in an exact set of governing equations for the crenulative turbulence. If the individual fluctuations of the instantaneous fluid variables could be calculated, the ensemble averaged Navier-Stokes equations would yield an "exact" solution to the flow. At this point in time there is no practical technique to calculate the individual fluctuations, so obtaining the "exact" governing equations is only an intermediate step in developing a model for crenulation turbulence.

Consider the incompressibility condition, equation (3.1), with a Reynolds decomposition and ensemble average of the instantaneous velocity

$$
\overline{\frac{\partial\left(\bar{u}_{l}+u_{l}^{\prime}\right)}{\partial x_{l}}}=0
$$

or

$$
\frac{\partial \bar{u}_{l}}{\partial x_{l}}+\frac{\partial \overline{u_{l}^{\prime}}}{\partial x_{l}}=0
$$

Because the ensemble average of a single fluctuating component is zero, the mean and fluctuational velocities are both solenodial, i.e. divergence free

$$
\frac{\partial \bar{u}_{l}}{\partial x_{l}}=0 \quad \& \quad \frac{\partial u_{l}^{\prime}}{\partial x_{l}}=0
$$

Consider the conservation of momentum, equation (3.2), with a Reynolds decomposition and ensemble average of the instantaneous velocity, pressure, and kinematic viscosity 


$$
\frac{\overline{\partial\left(\bar{u}_{i}+u_{i}^{\prime}\right)}}{\partial t}+\frac{\overline{\partial\left(\bar{u}_{i}+u_{i}^{\prime}\right)\left(\bar{u}_{l}+u_{l}^{\prime}\right)}}{\partial \dot{x}_{l}}=-\frac{\overline{\partial\left(\bar{p}+p^{\prime}\right)}}{\partial x_{i}}+\overline{\frac{\partial}{\partial x_{l}}\left[\left(\bar{v}+v^{\prime}\right)\left(\frac{\partial\left(\bar{u}_{i}+u_{i}^{\prime}\right)}{\partial x_{l}}+\frac{\partial\left(\bar{u}_{l}+u_{l}^{\prime}\right)}{\partial x_{i}}\right)\right]}
$$

which reduces to the following

$$
\frac{\partial \bar{u}_{i}}{\partial t}+\frac{\partial \bar{u}_{i} \bar{u}_{l}}{\partial x_{l}}+\frac{\partial \overline{u_{i}^{\prime} u_{l}^{\prime}}}{\partial x_{l}}=-\frac{\partial \bar{p}}{\partial x_{i}}+\frac{\partial}{\partial x_{l}}\left[\bar{v}\left(\frac{\partial \bar{u}_{i}}{\partial x_{l}}+\frac{\partial \bar{u}_{l}}{\partial x_{i}}\right)\right]+\frac{\partial}{\partial x_{l}}\left[\overline{v^{\prime} \frac{\partial u_{i}^{\prime}}{\partial x_{l}}+\overline{v^{\prime}} \frac{\partial u_{l}^{\prime}}{\partial x_{i}}}\right]
$$

Second order correlations due to the fluctuations of the instantaneous fluid variables are introduced. The quantity $\overline{u_{i}^{\prime} u_{l}^{\prime}}$ is a second order correlation known as the Reynolds stress tensor and is represented by $R_{i l}=\overline{u_{i}^{\prime} u_{l}^{\prime}}$. The quantity $\overline{v^{\prime} \frac{\partial u_{i}^{\prime}}{\partial x_{l}}}$ is also a second order correlation and can be rewritten as $\left(\frac{\partial \overline{v^{\prime} u_{i}^{\prime}}}{\partial x_{l}}-\overline{u_{i}^{\prime}} \frac{\partial v^{\prime}}{\partial x_{l}}\right)$. The two double correlations are represented by the vector $N_{i}=\overline{v^{\prime} u_{i}^{\prime}}$ and the second order tensor $M_{i l}=\overline{u_{i}^{\prime} \frac{\partial v^{\prime}}{\partial x_{l}}}$. With these definitions the mean momentum equation becomes

$$
\begin{gathered}
\frac{\partial \bar{u}_{i}}{\partial t}+\frac{\partial \bar{u}_{i} \bar{u}_{l}}{\partial x_{l}}+\frac{\partial R_{i l}}{\partial x_{l}}=-\frac{\partial \bar{p}}{\partial x_{i}}+\left(\frac{\partial \bar{u}_{i}}{\partial x_{l}}+\frac{\partial \bar{u}_{l}}{\partial x_{i}}\right) \frac{\partial \bar{v}}{\partial x_{l}}+\bar{v} \frac{\partial^{2} \bar{u}_{i}}{\partial x_{l}^{2}} \\
+\frac{\partial^{2} N_{i}}{\partial x_{l}^{2}}+\frac{\partial^{2} N_{l}}{\partial x_{l} \partial x_{i}}-\frac{\partial\left(M_{i l}+M_{l i}\right)}{\partial x_{l}}
\end{gathered}
$$

Consider the conservation of internal energy, equation (3.3), with a Reynolds decomposition and ensemble average of the instantaneous internal energy, velocity, and kinematic viscosity

$$
\overline{\frac{\partial\left(\bar{e}+e^{\prime}\right)}{\partial t}}+\overline{\frac{\partial\left(\bar{e}+e^{\prime}\right)\left(\bar{u}_{l}+u_{l}^{\prime}\right)}{\partial x_{l}}}=\overline{\left(\bar{v}+v^{\prime}\right)\left(\frac{\partial\left(\bar{u}_{i}+u_{i}^{\prime}\right)}{\partial x_{l}}+\frac{\partial\left(\bar{u}_{l}+u_{l}^{\prime}\right)}{\partial x_{i}}\right) \frac{\partial\left(\bar{u}_{i}+u_{i}^{\prime}\right)}{\partial x_{l}}}
$$


which reduces to the following

$$
\begin{aligned}
& \frac{\partial \bar{e}}{\partial t}+\frac{\partial \bar{e} \bar{u}_{l}}{\partial x_{l}}+\frac{\partial \overline{e^{\prime} u_{l}^{\prime}}}{\partial x_{l}}=\bar{v}\left(\frac{\partial \bar{u}_{i}}{\partial x_{l}}+\frac{\partial \bar{u}_{l}}{\partial x_{i}}\right) \frac{\partial \bar{u}_{i}}{\partial x_{l}}+\bar{v}\left(\overline{\frac{\partial u_{i}^{\prime}}{\partial x_{l}} \frac{\partial u_{i}^{\prime}}{\partial x_{l}}}+\frac{\overline{\partial u_{l}^{\prime}}}{\partial x_{i}} \frac{\partial u_{i}^{\prime}}{\partial x_{l}}\right) \\
& +\left(\overline{v^{\prime} \frac{\partial u_{i}^{\prime}}{\partial x_{l}}}+\overline{v^{\prime} \frac{\partial u_{l}^{\prime}}{\partial x_{i}}}\right) \frac{\partial \bar{u}_{i}}{\partial x_{l}}+\overline{v^{\prime} \frac{\partial u_{i}^{\prime}}{\partial x_{l}}}\left(\frac{\partial \bar{u}_{i}}{\partial x_{l}}+\frac{\partial \bar{u}_{l}}{\partial x_{i}}\right)+\overline{v^{\prime} \frac{\partial u_{i}^{\prime}}{\partial x_{l}} \frac{\partial u_{i}^{\prime}}{\partial x_{l}}}+\overline{v^{\prime} \frac{\partial u_{l}^{\prime}}{\partial x_{i}} \frac{\partial u_{i}^{\prime}}{\partial x_{l}}}
\end{aligned}
$$

New quantities due to the fluctuations of the instantaneous variables are introduced. The quantities $\overline{e^{\prime} u_{l}^{\prime}}, \frac{\overline{\partial u_{i}^{\prime}} \frac{\partial u_{i}^{\prime}}{\partial x_{l}}}{\partial x_{l}}$ and $\overline{\frac{\partial u_{l}^{\prime}}{\partial x_{i}} \frac{\partial u_{i}^{\prime}}{\partial x_{l}}}$ are second order correlations. The quantities $\overline{v^{\prime} \frac{\partial u_{i}^{\prime}}{\partial x_{l}}}$ and $\overline{v^{\prime} \frac{\partial u_{l}^{\prime}}{\partial x_{i}}}$ are the same second order tensor quantities as in equation (3.6). Notice that the mean internal energy equation introduces the quantities $\overline{v^{\prime} \frac{\partial u_{i}^{\prime}}{\partial x_{l}} \frac{\partial u_{i}^{\prime}}{\partial x_{l}}}$ and $\overline{v^{\prime} \frac{\partial u_{l}^{\prime}}{\partial x_{i}} \frac{\partial u_{i}^{\prime}}{\partial x_{l}}}$ which are triple correlations. In terms of the newly defined variables, the mean internal energy equation becomes

$$
\begin{aligned}
& \frac{\partial \bar{e}}{\partial t}+\frac{\partial \bar{e} \bar{u}_{l}}{\partial x_{l}}+\frac{\partial \overline{e^{\prime} u_{l}^{\prime}}}{\partial x_{l}}=\bar{v}\left(\frac{\partial \bar{u}_{i}}{\partial x_{l}}+\frac{\partial \bar{u}_{l}}{\partial x_{i}}\right) \frac{\partial \bar{u}_{i}}{\partial x_{l}}+\bar{v}\left(\overline{\frac{\partial u_{i}^{\prime}}{\partial x_{l}} \frac{\partial u_{i}^{\prime}}{\partial x_{l}}}+\frac{\partial^{2} R_{l i}}{\partial x_{l} \partial x_{i}}\right) \\
& +\left(\frac{\partial N_{i}}{\partial x_{l}}-M_{i l}+\frac{\partial N_{l}}{\partial x_{i}}-M_{l i}\right) \frac{\partial \bar{u}_{i}}{\partial x_{l}}+\left(\frac{\partial N_{i}}{\partial x_{l}}-M_{i l}\right)\left(\frac{\partial \bar{u}_{i}}{\partial x_{l}}+\frac{\partial \bar{u}_{l}}{\partial x_{i}}\right) \\
& +\overline{v^{\prime} \frac{\partial u_{i}^{\prime}}{\partial x_{l}} \frac{\partial u_{i}^{\prime}}{\partial x_{l}}}+\overline{v^{\prime} \frac{\partial u_{l}^{\prime}}{\partial x_{i}} \frac{\partial u_{i}^{\prime}}{\partial x_{l}}}
\end{aligned}
$$

Consider the evolution of the kinematic viscosity, equation (3.4), with a Reynolds decomposition and ensemble average of the instantaneous velocity and kinematic viscosity

or

$$
\begin{gathered}
\frac{\overline{\partial\left(\bar{v}+v^{\prime}\right)}}{\partial t}+\frac{\overline{\partial\left(\bar{v}+v^{\prime}\right)\left(\bar{u}_{l}+u_{l}^{\prime}\right)}}{\partial x_{l}}=0 \\
\frac{\partial \bar{v}}{\partial t}+\frac{\partial \bar{v} \bar{u}_{l}}{\partial x_{l}}+\frac{\partial \overline{v^{\prime} u_{l}^{\prime}}}{\partial x_{l}}=0
\end{gathered}
$$


Because both the mean and fluctuational velocities are solenodial, the evolution of the mean kinematic viscosity can be written as

$$
\text { or } \quad \begin{aligned}
& \frac{\partial \bar{v}}{\partial t}+\bar{u}_{l} \frac{\partial \bar{v}}{\partial x_{l}}+\overline{u_{l}^{\prime} \frac{\partial v^{\prime}}{\partial x_{l}}}=0 \\
& \frac{\partial \bar{v}}{\partial t}+\bar{u}_{l} \frac{\partial \bar{v}}{\partial x_{l}}+\frac{\partial \overline{v^{\prime} u_{l}^{\prime}}}{\partial x_{l}}=0
\end{aligned}
$$

The quantity $\overline{u_{l}^{\prime} \frac{\partial v^{\prime}}{\partial x_{l}}}$ is the contraction of the second order tensor $M_{i l}$, hence the evolution of the mean kinematic viscosity becomes

or

$$
\begin{aligned}
& \frac{\partial \bar{v}}{\partial t}+\bar{u}_{l} \frac{\partial \bar{v}}{\partial x_{l}}+M_{l l}=0 \\
& \frac{\partial \bar{v}}{\partial t}+\bar{u}_{l} \frac{\partial \bar{v}}{\partial x_{l}}+\frac{\partial N_{l}}{\partial x_{l}}=0
\end{aligned}
$$

Equations (3.5), (3.6), (3.7), and (3.8) are the exact equations for conservation of mass, mean momentum, mean internal energy, and evolution of the mean kinematic viscosity that govern the crenulative flow. A Reynolds decomposition and ensemble average of the basic governing equations may make the governing equations appear more complicated, but they reveal the effects due to mean behavior and turbulent behavior. It is evident that the turbulence quantities $R_{i l}$ and $M_{i l}$ play vital roles in the evolution of the mean momentum and the mean internal energy. So what do we do with these higher-order moments and how do we calculate them? We can do several things: we might use a Boussinesq approximation technique; we might model these quantities with other known quantities (such as $\bar{u}_{i}, \bar{p}$, and $\bar{v}$ ); we might simply neglect them; or we might develop transport (or evolution) equations for them. We choose the last of these possibilities, 
because the information obtained from a transport equation gives us much more insight to the crenulative behavior than any other method. 


\section{Chapter 4}

\section{Exact Transport Equations in Cartesian Tensor Form}

In this chapter the exact transport equations for the Reynolds stress, $R_{i j}$, the $M_{i j}$ tensor, the $N_{i}$ vector, and the $Z_{i j}$ tensor (which comes from the transport equation for $M_{i j}$ ) are derived. The Reynolds stress tensor is derived by taking a "moment" of the basic (unaveraged) equation for conservation of momentum. The $M_{i j}$ tensor is derived by taking moments of the equations for conservation of momentum and the evolution of the kinematic viscosity. The $Z_{i j}$ tensor transport is derived by taking a moment of the evolution equation for the kinematic viscosity. Also, in this chapter the different modes of energy are discussed, and the evolution equations for mean kinetic energy and turbulent kinetic energy are derived.

\subsection{Reynolds Stress Transport Equation Derivation}

The basic (unaveraged) momentum equation is the foundation for deriving a transport equation for the Reynolds stress tensor $R_{i j}$. The first step is to multiply the momentum equation by $u_{j}^{\prime}$

$$
u_{j}^{\prime}\left\{\frac{\partial u_{i}}{\partial t}+u_{l} \frac{\partial u_{i}}{\partial x_{l}}=-\frac{\partial p}{\partial x_{i}}+\frac{\partial}{\partial x_{l}}\left[v\left(\frac{\partial u_{i}}{\partial x_{l}}+\frac{\partial u_{l}}{\partial x_{i}}\right)\right]\right\}
$$

The second step is to make a Reynolds decomposition and take an ensemble average of this equation 


$$
\begin{aligned}
& \overline{u_{j}^{\prime} \frac{\partial u_{i}^{\prime}}{\partial t}}+\overline{u_{j}^{\prime} u_{l}^{\prime}} \frac{\partial \bar{u}_{i}}{\partial x_{l}}+\overline{u_{l} u_{j}^{\prime} \frac{\partial u_{i}^{\prime}}{\partial x_{l}}}+\overline{u_{j}^{\prime} u_{l}^{\prime} \frac{\partial u_{i}^{\prime}}{\partial x_{l}}}=\overline{-u_{j}^{\prime} \frac{\partial p^{\prime}}{\partial x_{i}}}+\overline{u_{j}^{\prime} \frac{\partial}{\partial x_{l}\left[v^{\prime}\left(\frac{\partial \bar{u}_{i}}{\partial x_{l}}+\frac{\partial \bar{u}_{l}}{\partial x_{i}}\right)\right]}} \\
& +\overline{u_{j}^{\prime} \frac{\partial}{\partial x_{l}}\left[\bar{v}\left(\frac{\partial u_{i}^{\prime}}{\partial x_{l}}+\frac{\partial u_{l}^{\prime}}{\partial x_{i}}\right)\right]}+\overline{u_{j}^{\prime} \frac{\partial}{\partial x_{l}}\left[v^{\prime}\left(\frac{\partial u_{i}^{\prime}}{\partial x_{l}}+\frac{\partial u_{l}^{\prime}}{\partial x_{i}}\right)\right]}
\end{aligned}
$$

The third step is to reverse the free indices $i$ and $j$ in equation (4.2) to obtain a companion equation

$$
\begin{aligned}
& \overline{u_{i}^{\prime} \frac{\partial u_{j}^{\prime}}{\partial t}}+\overline{u_{i}^{\prime} u_{l}^{\prime}} \frac{\partial \bar{u}_{j}}{\partial x_{l}}+\overline{u_{l} u_{i}^{\prime} \frac{\partial u_{j}^{\prime}}{\partial x_{l}}}+\overline{u_{i}^{\prime} u_{l}^{\prime} \frac{\partial u_{j}^{\prime}}{\partial x_{l}}}=\overline{-u_{i}^{\prime} \frac{\partial p^{\prime}}{\partial x_{j}}}+\overline{u_{i}^{\prime} \frac{\partial}{\partial x_{l}}\left[v^{\prime}\left(\frac{\partial \bar{u}_{j}}{\partial x_{l}}+\frac{\partial \bar{u}_{l}}{\partial x_{j}}\right)\right]} \\
& +u_{i}^{\prime} \frac{\partial}{\partial x_{l}}\left[\bar{v}\left(\frac{\partial u_{j}^{\prime}}{\partial x_{l}}+\frac{\partial u_{l}^{\prime}}{\partial x_{j}}\right)\right]+\overline{u_{i}^{\prime} \frac{\partial}{\partial x_{l}}\left[v^{\prime}\left(\frac{\partial u_{j}^{\prime}}{\partial x_{l}}+\frac{\partial u_{l}^{\prime}}{\partial x_{j}}\right)\right]}
\end{aligned}
$$

The fourth step is to add equations (4.2) and (4.3), and combine terms using the product rule

$$
\begin{aligned}
& \frac{\partial \overline{u_{i}^{\prime} u_{j}^{\prime}}}{\partial t}+\overline{u_{j}^{\prime} u_{l}^{\prime}} \frac{\partial \bar{u}_{i}}{\partial x_{l}}+\overline{u_{i}^{\prime} u_{l}^{\prime}} \frac{\partial \bar{u}_{j}}{\partial x_{l}}+\bar{u}_{l} \frac{\partial \overline{u_{i}^{\prime} u_{j}^{\prime}}}{\partial x_{l}}+\overline{\frac{\partial u_{i}^{\prime} u_{j}^{\prime} u_{l}^{\prime}}{\partial x_{l}}}=-\overline{u_{j}^{\prime} \frac{\partial p^{\prime}}{\partial x_{i}}}-\overline{u_{i}^{\prime} \frac{\partial p^{\prime}}{\partial x_{j}}}
\end{aligned}
$$

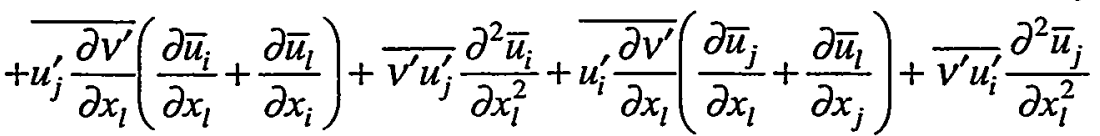

$$
\begin{aligned}
& +\left(\overline{u_{j}^{\prime} \frac{\partial u_{i}^{\prime}}{\partial x_{l}}}+\overline{u_{j}^{\prime} \frac{\partial u_{l}^{\prime}}{\partial x_{i}}}\right) \frac{\partial \bar{v}}{\partial x_{l}}+\left(\overline{u_{i}^{\prime} \frac{\partial u_{j}^{\prime}}{\partial x_{l}}}+\overline{u_{i}^{\prime} \frac{\partial u_{l}^{\prime}}{\partial x_{j}}}\right) \frac{\partial \bar{v}}{\partial x_{l}}+\bar{v}\left[\frac{\partial^{2} \overline{u_{i}^{\prime} u_{j}^{\prime}}}{\partial x_{l}^{2}}-2 \overline{\frac{\partial u_{i}^{\prime}}{\partial x_{l}} \frac{\partial u_{j}^{\prime}}{\partial x_{l}}}\right] \\
& +\overline{+u_{j}^{\prime} \frac{\partial}{\partial x_{l}}\left[v^{\prime}\left(\frac{\partial u_{i}^{\prime}}{\partial x_{l}}+\frac{\partial u_{l}^{\prime}}{\partial x_{i}}\right)\right]}+\overline{u_{i}^{\prime} \frac{\partial}{\partial x_{l}}\left[v^{\prime}\left(\frac{\partial u_{j}^{\prime}}{\partial x_{l}}+\frac{\partial u_{l}^{\prime}}{\partial x_{j}}\right)\right]}
\end{aligned}
$$

We can see there are Reynolds stress terms, triple correlations, pressure correlations, $M_{i j}$ terms, $N_{i}$ terms, and various other moments. Replacing some of the quantities with their newly defined terms we get the following transport equation, which describes the variations of Reynolds stress 


$$
\begin{gathered}
\frac{\partial R_{i j}}{\partial t}+R_{j l} \frac{\partial \bar{u}_{i}}{\partial x_{l}}+R_{i l} \frac{\partial \bar{u}_{j}}{\partial x_{l}}+\bar{u}_{l} \frac{\partial R_{i j}}{\partial x_{l}}+\frac{\overline{\partial u_{i}^{\prime} u_{j}^{\prime} u_{l}^{\prime}}}{\partial x_{l}}=-\overline{u_{j}^{\prime} \frac{\partial p^{\prime}}{\partial x_{i}}}-\overline{\overline{u_{i}^{\prime}} \frac{\partial p^{\prime}}{\partial x_{j}}} \\
+M_{j l}\left(\frac{\partial \bar{u}_{i}}{\partial x_{l}}+\frac{\partial \bar{u}_{l}}{\partial x_{i}}\right)+N_{j} \frac{\partial^{2} \bar{u}_{i}}{\partial x_{l}^{2}}+M_{i l}\left(\frac{\partial \bar{u}_{j}}{\partial x_{l}}+\frac{\partial \bar{u}_{l}}{\partial x_{j}}\right)+N_{i} \frac{\partial^{2} \bar{u}_{j}}{\partial x_{l}^{2}} \\
+\left(\frac{\partial R_{i j}}{\partial x_{l}}+\overline{\left.u_{j}^{\prime} \frac{\partial u_{l}^{\prime}}{\partial x_{i}}+\overline{u_{i}^{\prime} \frac{\partial u_{l}^{\prime}}{\partial x_{j}}}\right) \frac{\partial \bar{v}}{\partial x_{l}}+\bar{v} \frac{\partial^{2} R_{i j}}{\partial x_{l}^{2}}-2 \bar{v} \frac{\partial u_{i}^{\prime}}{\partial x_{l}} \frac{\partial u_{j}^{\prime}}{\partial x_{l}}}\right. \\
+\quad+u_{j}^{\prime} \frac{\partial}{\partial x_{l}}\left[v^{\prime}\left(\frac{\partial u_{i}^{\prime}}{\partial x_{l}}+\frac{\partial u_{l}^{\prime}}{\partial x_{i}}\right)\right]+u_{i}^{\prime} \frac{\partial}{\partial x_{l}}\left[v^{\prime}\left(\frac{\partial u_{j}^{\prime}}{\partial x_{l}}+\frac{\partial u_{l}^{\prime}}{\partial x_{j}}\right)\right]
\end{gathered}
$$

This is an exact transport equation of the Reynolds stress tensor, in which some new turbulence quantities arise because of the fluctuational velocity moment used to derive this transport equation. All the second and third order correlations need to be modeled or a transport equation needs to be developed for them. In equation (4.4) there are contributions to the Reynolds stress tensor from the $M_{i j}$ tensor and the $N_{i}$ vector coupled to the mean flow gradient. It is therefore appropriate to develop transport equations for both $M_{i j}$ and $N_{i}$.

\section{2 $M_{i j}$ Transport Derivation}

The basic (unaveraged) momentum equation and the evolution equation for kinematic viscosity are the foundations for deriving a transport equation for $M_{i j}$. The first step is multiplying the momentum equation by $\frac{\partial v^{\prime}}{\partial x_{j}}$

$$
\frac{\partial v^{\prime}}{\partial x_{j}}\left\{\frac{\partial u_{i}}{\partial t}+u_{l} \frac{\partial u_{i}}{\partial x_{l}}=-\frac{\partial p}{\partial x_{i}}+\frac{\partial}{\partial x_{k}}\left[v\left(\frac{\partial u_{i}}{\partial x_{k}}+\frac{\partial u_{k}}{\partial x_{i}}\right)\right]\right\}
$$

The second step is to make Reynolds decomposition and take an ensemble average of this equation 


$$
\begin{aligned}
& \overline{\frac{\partial v^{\prime}}{\partial x_{j}} \frac{\partial u_{i}^{\prime}}{\partial t}}+\overline{u_{l}^{\prime} \frac{\partial v^{\prime}}{\partial x_{j}}} \frac{\partial \bar{u}_{i}}{\partial x_{l}}+\overline{u_{l}} \overline{\frac{\partial v^{\prime}}{\partial x_{j}} \frac{\partial u_{i}^{\prime}}{\partial x_{l}}}+\overline{u_{l}^{\prime} \frac{\partial v^{\prime}}{\partial x_{j}} \frac{\partial u_{i}^{\prime}}{\partial x_{l}}} \\
& =-\overline{\frac{\partial v^{\prime}}{\partial x_{j}} \frac{\partial p^{\prime}}{\partial x_{i}}}+\overline{\frac{\partial v^{\prime}}{\partial x_{j}} \frac{\partial}{\partial x_{k}}\left[v^{\prime}\left(\frac{\partial \bar{u}_{i}}{\partial x_{k}}+\frac{\partial \bar{u}_{k}}{\partial x_{i}}\right)\right]} \\
& \overline{+\frac{\partial v^{\prime}}{\partial x_{j}} \frac{\partial}{\partial x_{k}}\left[\bar{v}\left(\frac{\partial u_{i}^{\prime}}{\partial x_{k}}+\frac{\partial u_{k}^{\prime}}{\partial x_{i}}\right)\right]}+\overline{\frac{\partial v^{\prime}}{\partial x_{j}} \frac{\partial}{\partial x_{k}}\left[v^{\prime}\left(\frac{\partial u_{i}^{\prime}}{\partial x_{k}}+\frac{\partial u_{k}^{\prime}}{\partial x_{i}}\right)\right]}
\end{aligned}
$$

The third step is to take the gradient of the evolution of the kinematic viscosity and multiply by $u_{i}^{\prime}$

$$
u_{i}^{\prime} \frac{\partial}{\partial x_{j}}\left\{\frac{\partial v}{\partial t}+u_{l} \frac{\partial v}{\partial x_{l}}=0\right\}
$$

The fourth step is to make a Reynolds decomposition and take an ensemble average of this equation

$$
\overline{u_{i}^{\prime} \frac{\partial}{\partial x_{j}}\left(\frac{\partial v^{\prime}}{\partial t}\right)}+\overline{u_{i}^{\prime} \frac{\partial}{\partial x_{j}}\left(u_{l}^{\prime} \frac{\partial \bar{v}}{\partial x_{l}}\right)}+\overline{u_{i}^{\prime} \frac{\partial}{\partial x_{j}}\left(\bar{u}_{l} \frac{\partial v^{\prime}}{\partial x_{l}}\right)}+\overline{u_{i}^{\prime} \frac{\partial}{\partial x_{j}}\left(u_{l}^{\prime} \frac{\partial v^{\prime}}{\partial x_{l}}\right)}=0
$$

The first term $\overline{u_{i}^{\prime} \frac{\partial}{\partial x_{j}}\left(\frac{\partial v^{\prime}}{\partial t}\right)}$ can be rewritten as $\overline{u_{i}^{\prime} \frac{\partial}{\partial t}\left(\frac{\partial v^{\prime}}{\partial x_{j}}\right)}$, hence we get the following

$$
\overline{u_{i}^{\prime} \frac{\partial}{\partial t}\left(\frac{\partial v^{\prime}}{\partial x_{j}}\right)}+\overline{u_{i}^{\prime} \frac{\partial}{\partial x_{j}}\left(u_{l}^{\prime} \frac{\partial \bar{v}}{\partial x_{l}}\right)}+\overline{u_{i}^{\prime} \frac{\partial}{\partial x_{j}}\left(\bar{u}_{l} \frac{\partial v^{\prime}}{\partial x_{l}}\right)}+\overline{u_{i}^{\prime} \frac{\partial}{\partial x_{j}}\left(u_{l}^{\prime} \frac{\partial v^{\prime}}{\partial x_{l}}\right)}=0
$$

The fifth step is to add equations (4.6) and (4.9), and combine terms 


$$
\begin{aligned}
& \overline{\frac{\partial}{\partial t}\left(u_{i}^{\prime} \frac{\partial v^{\prime}}{\partial x_{j}}\right)}+\overline{u_{l}^{\prime} \frac{\partial v^{\prime}}{\partial x_{j}}} \frac{\partial \bar{u}_{i}}{\partial x_{l}}+\overline{u_{i}^{\prime} \frac{\partial u_{l}^{\prime}}{\partial x_{j}}} \frac{\partial \bar{v}}{\partial x_{l}}+\overline{u_{i}^{\prime} u_{l}^{\prime}} \frac{\partial^{2} \bar{v}}{\partial x_{l}^{2}}+\bar{u}_{l} \frac{\partial v^{\prime}}{\partial x_{j}} \frac{\partial u_{i}^{\prime}}{\partial x_{l}}+\overline{u_{i}^{\prime}} \frac{\partial v^{\prime}}{\partial x_{l}} \frac{\partial \bar{u}_{l}}{\partial x_{j}} \\
& +\bar{u}_{l} \frac{\partial}{\partial x_{l}}\left(\overline{u_{i}^{\prime} \frac{\partial v^{\prime}}{\partial x_{j}}}\right)-\bar{u}_{l} \frac{\overline{\partial u_{i}^{\prime}}}{\partial x_{l} \frac{\partial v^{\prime}}{\partial x_{j}}}+\overline{u_{l}^{\prime} \frac{\partial v^{\prime}}{\partial x_{j}} \frac{\partial u_{i}^{\prime}}{\partial x_{l}}}+\overline{u_{i}^{\prime} \frac{\partial}{\partial x_{j}}\left(u_{l}^{\prime} \frac{\partial v^{\prime}}{\partial x_{l}}\right)} \\
& =-\overline{\frac{\partial v^{\prime}}{\partial x_{j}} \frac{\partial p^{\prime}}{\partial x_{i}}}+\overline{\frac{\partial v^{\prime}}{\partial x_{j}} \frac{\partial v^{\prime}}{\partial x_{k}}}\left(\frac{\partial \bar{u}_{i}}{\partial x_{k}}+\frac{\partial \bar{u}_{k}}{\partial x_{i}}\right)+\overline{v^{\prime} \frac{\partial v^{\prime}}{\partial x_{j}} \frac{\partial^{2} \bar{u}_{i}}{\partial x_{k}^{2}}} \\
& +\left(\overline{\frac{\partial v^{\prime}}{\partial x_{j}} \frac{\partial u_{i}^{\prime}}{\partial x_{k}}}+\overline{\frac{\partial v^{\prime}}{\partial x_{j}} \frac{\partial u_{k}^{\prime}}{\partial x_{i}}}\right) \frac{\partial \bar{v}}{\partial x_{k}}+\bar{v} \frac{\partial v^{\prime}}{\partial x_{j}} \frac{\partial^{2} u_{i}^{\prime}}{\partial x_{k}^{2}} \\
& \overline{+\frac{\partial v^{\prime}}{\partial x_{j}} \frac{\partial v^{\prime}}{\partial x_{k}}\left(\frac{\partial u_{i}^{\prime}}{\partial x_{k}}+\frac{\partial u_{k}^{\prime}}{\partial x_{i}}\right)}+\overline{v^{\prime} \frac{\partial v^{\prime}}{\partial x_{j}} \frac{\partial^{2} u_{i}^{\prime}}{\partial x_{k}^{2}}}
\end{aligned}
$$

We can see there are $M_{i j}$ terms, Reynolds stress $R_{i j}$ terms, and various new double and triple correlations. The term $\frac{\partial v^{\prime}}{\partial x_{j}} \frac{\partial v^{\prime}}{\partial x_{k}}$ is a second order correlation, which describes the configurational state of the nonhomogeneities and is represented by $Z_{j k}=\frac{\overline{\partial v^{\prime}} \frac{\partial v^{\prime}}{\partial x_{j}}}{\partial x_{k}}$.

Replacing some of the moments with the newly defined terms we get the following transport equation for $M_{i j}$

$$
\begin{gathered}
\frac{\partial M_{i j}}{\partial t}+M_{l j} \frac{\partial \bar{u}_{i}}{\partial x_{l}}+\overline{u_{i}^{\prime} \frac{\partial u_{l}^{\prime}}{\partial x_{j}} \frac{\partial \bar{v}}{\partial x_{l}}}+R_{i l} \frac{\partial^{2} \bar{v}}{\partial x_{l}^{2}}+M_{i l} \frac{\partial \bar{u}_{l}}{\partial x_{j}}+\bar{u}_{l} \frac{\partial M_{i j}}{\partial x_{l}} \\
+\overline{u_{l}^{\prime} \frac{\partial v^{\prime}}{\partial x_{j}} \frac{\partial u_{i}^{\prime}}{\partial x_{l}}+\overline{u_{i}^{\prime}} \frac{\partial}{\partial x_{j}}\left(u_{l}^{\prime} \frac{\partial v^{\prime}}{\partial x_{l}}\right)}=-\frac{\overline{\partial v^{\prime}} \frac{\partial p^{\prime}}{\partial x_{j}}}{\partial x_{i}}+Z_{j k}\left(\frac{\partial \bar{u}_{i}}{\partial x_{k}}+\frac{\partial \bar{u}_{k}}{\partial x_{i}}\right) \\
+\frac{1}{2} \frac{\partial \overline{v^{\prime} v^{\prime}}}{\partial x_{j}} \frac{\partial^{2} \bar{u}_{i}}{\partial x_{k}^{2}}+\left(\frac{\left.\overline{\partial v^{\prime}} \frac{\partial u_{i}^{\prime}}{\partial x_{j}}+\frac{\partial v^{\prime}}{\partial x_{k}} \frac{\partial u_{k}^{\prime}}{\partial x_{j}}\right)}{\partial x_{i}}\right) \frac{\partial \bar{v}}{\partial x_{k}}+\bar{v} \frac{\partial^{2} M_{i j}}{\partial x_{l}^{2}}-2 \bar{v} \frac{\partial u_{i}^{\prime}}{\partial x_{l}} \frac{\partial^{2} v^{\prime}}{\partial x_{l} \partial x_{j}} \\
-\bar{v} u_{i}^{\prime} \frac{\partial^{3} v^{\prime}}{\partial x_{l}^{2} \partial x_{j}}+\frac{\partial v^{\prime}}{\partial x_{j}} \frac{\partial v^{\prime}}{\partial x_{k}}\left(\frac{\partial u_{i}^{\prime}}{\partial x_{k}}+\frac{\partial u_{k}^{\prime}}{\partial x_{i}}\right)+\frac{v^{\prime} \frac{\partial v^{\prime}}{\partial x_{j}} \frac{\partial^{2} u_{i}^{\prime}}{\partial x_{k}^{2}}}{}
\end{gathered}
$$

This is the exact transport equation for $M_{i j}$, which contains yet more moments. All the second and third order correlations not previously defined need to be modeled or transport equations need to be developed for them. In equation (4.4) there is a contribution to the 
Reynolds stress tensor $R_{i j}$ from $M_{i j}$ coupled to the mean flow gradients, and because of its importance we developed a transport equation for $M_{i j}$. In equation (4.10) there is a contribution to $M_{i j}$ from the $Z_{i j}$ coupled to the mean flow gradients, and because of its importance we develop a transport equation for $Z_{i j}$, but first we developed a transport equation for $N_{i}$.

\section{3 $N_{i}$ Transport Derivation}

The basic (unaveraged) momentum equation and the evolution equation for kinematic viscosity are the foundations for deriving a transport equation for $N_{i}$. The first step is multiplying the momentum equation by $v^{\prime}$

$$
v^{\prime}\left\{\frac{\partial u_{i}}{\partial t}+u_{l} \frac{\partial u_{i}}{\partial x_{l}}=-\frac{\partial p}{\partial x_{i}}+\frac{\partial}{\partial x_{k}}\left[v\left(\frac{\partial u_{i}}{\partial x_{k}}+\frac{\partial u_{k}}{\partial x_{i}}\right)\right]\right\}
$$

The second step is to make Reynolds decomposition and take an ensemble average of this equation

$$
\begin{aligned}
\overline{v^{\prime} \frac{\partial u_{i}^{\prime}}{\partial t}}+\overline{v^{\prime} u_{l}^{\prime}} \frac{\partial \bar{u}_{i}}{\partial x_{l}}+\overline{u_{l}} \overline{v^{\prime} \frac{\partial u_{i}^{\prime}}{\partial x_{l}}}+\overline{v^{\prime} u_{l}^{\prime} \frac{\partial u_{i}^{\prime}}{\partial x_{l}}}=-\overline{v^{\prime} \frac{\partial p^{\prime}}{\partial x_{i}}}+\overline{v^{\prime} \frac{\partial}{\partial x_{k}}\left[v^{\prime}\left(\frac{\partial \bar{u}_{i}}{\partial x_{k}}+\frac{\partial \bar{u}_{k}}{\partial x_{i}}\right)\right]} \\
+v^{\prime} \frac{\partial}{\partial x_{k}}\left[\bar{v}\left(\frac{\partial u_{i}^{\prime}}{\partial x_{k}}+\frac{\partial u_{k}^{\prime}}{\partial x_{i}}\right)\right]+v^{\prime} \frac{\partial}{\partial x_{k}\left[v^{\prime}\left(\frac{\partial u_{i}^{\prime}}{\partial x_{k}}+\frac{\partial u_{k}^{\prime}}{\partial x_{i}}\right)\right]}
\end{aligned}
$$

The third step is to take the evolution of the kinematic viscosity and multiply by $u_{i}^{\prime}$

$$
u_{i}^{\prime}\left\{\frac{\partial v}{\partial t}+u_{l} \frac{\partial v}{\partial x_{l}}=0\right\}
$$


The fourth step is to make Reynolds decomposition and take an ensemble average of this equation

$$
\overline{u_{i}^{\prime} \frac{\partial v^{\prime}}{\partial t}}+\overline{u_{i}^{\prime} u_{l}^{\prime} \frac{\partial \bar{v}}{\partial x_{l}}}+\overline{u_{l} u_{i}^{\prime} \frac{\partial v^{\prime}}{\partial x_{l}}}+\overline{u_{i}^{\prime} u_{l}^{\prime} \frac{\partial v^{\prime}}{\partial x_{l}}}=0
$$

The fifth step is to add equations (4.12) and (4.14), and combine terms

$$
\frac{\frac{\partial \overline{u_{i}^{\prime} v^{\prime}}}{\partial t}+\overline{v^{\prime} u_{l}^{\prime}} \frac{\partial \bar{u}_{i}}{\partial x_{l}}+\bar{u}_{l} \frac{\partial \overline{u_{i}^{\prime} v^{\prime}}}{\partial x_{l}}+\overline{u_{i}^{\prime} u_{l}^{\prime}} \frac{\partial \bar{v}}{\partial x_{l}}+\frac{\partial \overline{u_{i}^{\prime} v^{\prime} u_{l}^{\prime}}}{\partial x_{l}}=-\overline{v^{\prime} \frac{\partial p^{\prime}}{\partial x_{i}}}}{+v^{\prime} \frac{\partial}{\partial x_{k}}\left[v^{\prime}\left(\frac{\partial \bar{u}_{i}}{\partial x_{k}}+\frac{\partial \bar{u}_{k}}{\partial x_{i}}\right)\right]+v^{\prime} \frac{\partial}{\partial x_{k}}\left[\bar{v}\left(\frac{\partial u_{i}^{\prime}}{\partial x_{k}}+\frac{\partial u_{k}^{\prime}}{\partial x_{i}}\right)\right]+v^{\prime} \frac{\partial}{\partial x_{k}}\left[v^{\prime}\left(\frac{\partial u_{i}^{\prime}}{\partial x_{k}}+\frac{\partial u_{k}^{\prime}}{\partial x_{i}}\right)\right]}
$$

We can see there are $N_{i}$ terms, Reynolds stress $R_{i j}$ terms, and various new double and triple correlations. Replacing some of the moments with the newly defined terms we get the following transport equation for $N_{i}$

$$
\begin{gathered}
\frac{\partial N_{i}}{\partial t}+N_{l} \frac{\partial \bar{u}_{i}}{\partial x_{l}}+\bar{u}_{l} \frac{\partial N_{i}}{\partial x_{l}}+R_{i l} \frac{\partial \bar{v}}{\partial x_{l}}+\frac{\partial \overline{u_{i}^{\prime} v^{\prime} u^{\prime}}}{\partial x_{l}}=-\overline{v^{\prime} \frac{\partial p^{\prime}}{\partial x_{i}}} \\
+\frac{1}{2} \frac{\partial \overline{v^{\prime} v^{\prime}}}{\partial x_{k}}\left(\frac{\partial \bar{u}_{i}}{\partial x_{k}}+\frac{\partial \bar{u}_{k}}{\partial x_{i}}\right)+\overline{v^{\prime} v^{\prime}} \frac{\partial^{2} \bar{u}_{i}}{\partial x_{k}^{2}}+\left(\frac{\partial N_{i}}{\partial x_{k}}-M_{i k}+\frac{\partial N_{k}}{\partial x_{i}}-M_{k i}\right) \frac{\partial \bar{v}}{\partial x_{k}} \\
+\bar{v} \frac{\partial^{2} N_{i}}{\partial x_{k}^{2}}-\bar{v} \frac{\partial M_{i k}}{\partial x_{k}}-\bar{v} \frac{\partial v^{\prime}}{\partial x_{k}} \frac{\partial u_{i}^{\prime}}{\partial x_{k}}+v^{\prime} \frac{\partial}{\partial x_{k}}\left[v^{\prime}\left(\frac{\partial u_{i}^{\prime}}{\partial x_{k}}+\frac{\partial u_{k}^{\prime}}{\partial x_{i}}\right)\right]
\end{gathered}
$$

This is the exact transport equation for $N_{i}$, which contains yet more moments. All the second and third order correlations not previously defined need to be modeled or transport equations need to be developed for them. 


\section{4 $Z_{i j}$ Transport Derivation}

The basic (unaveraged) equation for evolution of the kinematic viscosity is the foundation for deriving a transport equation for $Z_{i j}$. The first step is to take the gradient of the evolution equation for the kinematic viscosity and then multiply by $\frac{\partial v^{\prime}}{\partial x_{i}}$

$$
\frac{\partial v^{\prime}}{\partial x_{i}} \frac{\partial}{\partial x_{j}}\left\{\frac{\partial v}{\partial t}+u_{l} \frac{\partial v}{\partial x_{l}}=0\right\}
$$

The second step is to make a Reynolds decomposition and take an ensemble average of this equation

$$
\begin{aligned}
& \overline{\frac{\partial v^{\prime}}{\partial x_{i}} \frac{\partial}{\partial x_{j}}\left(\frac{\partial v^{\prime}}{\partial t}\right)}+\overline{\frac{\partial v^{\prime}}{\partial x_{i}} \frac{\partial}{\partial x_{j}}\left(\bar{u}_{l} \frac{\partial v^{\prime}}{\partial x_{l}}\right)} \\
& +\overline{\frac{\partial v^{\prime}}{\partial x_{i}} \frac{\partial}{\partial x_{j}}\left(u_{l}^{\prime} \frac{\partial \bar{v}}{\partial x_{l}}\right)}+\overline{\frac{\partial v^{\prime}}{\partial x_{i}} \frac{\partial}{\partial x_{j}}\left(u_{l}^{\prime} \frac{\partial v^{\prime}}{\partial x_{l}}\right)}=0
\end{aligned}
$$

The first term $\overline{\frac{\partial v^{\prime}}{\partial x_{i}} \frac{\partial}{\partial x_{j}}\left(\frac{\partial v^{\prime}}{\partial t}\right)}$ can be rewritten as $\overline{\frac{\partial v^{\prime}}{\partial x_{i}} \frac{\partial}{\partial t}\left(\frac{\partial v^{\prime}}{\partial x_{j}}\right)}$, hence we get the following equation

$$
\begin{aligned}
& \overline{\frac{\partial v^{\prime}}{\partial x_{i}} \frac{\partial}{\partial t}\left(\frac{\partial v^{\prime}}{\partial x_{j}}\right)}+\overline{\frac{\partial v^{\prime}}{\partial x_{i}} \frac{\partial}{\partial x_{j}}\left(\bar{u}_{l} \frac{\partial v^{\prime}}{\partial x_{l}}\right)} \\
& \overline{+\frac{\partial v^{\prime}}{\partial x_{i}} \frac{\partial}{\partial x_{j}}\left(u_{l}^{\prime} \frac{\partial \bar{v}}{\partial x_{l}}\right)}+\overline{\frac{\partial v^{\prime}}{\partial x_{i}} \frac{\partial}{\partial x_{j}}\left(u_{l}^{\prime} \frac{\partial v^{\prime}}{\partial x_{l}}\right)}=0
\end{aligned}
$$

The third step is to reverse the free indices $i$ and $j$ in equation (4.18) to obtain a companion equation 


$$
\begin{aligned}
& \overline{\frac{\partial v^{\prime}}{\partial x_{j}} \frac{\partial}{\partial t}\left(\frac{\partial v^{\prime}}{\partial x_{i}}\right)}+\overline{\frac{\partial v^{\prime}}{\partial x_{j}} \frac{\partial}{\partial x_{i}}\left(\bar{u}_{l} \frac{\partial v^{\prime}}{\partial x_{l}}\right)} \\
& +\overline{\frac{\partial v^{\prime}}{\partial x_{j}} \frac{\partial}{\partial x_{i}}\left(u_{l}^{\prime} \frac{\partial \bar{v}}{\partial x_{l}}\right)}+\overline{\frac{\partial v^{\prime}}{\partial x_{j}} \frac{\partial}{\partial x_{i}}\left(u_{l}^{\prime} \frac{\partial v^{\prime}}{\partial x_{l}}\right)}=0
\end{aligned}
$$

The fourth step is to add equations (4.18) and (4.19) and combine terms

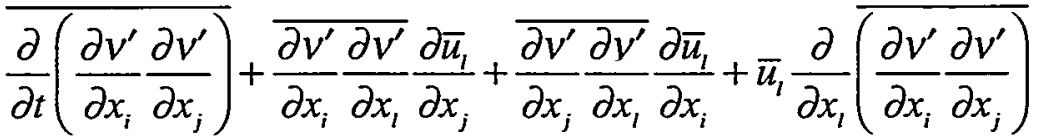

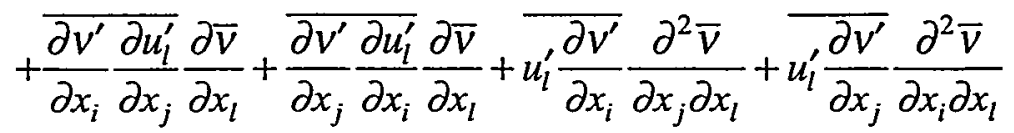

$$
\begin{aligned}
& +\overline{\frac{\partial v^{\prime}}{\partial x_{i}} \frac{\partial u_{l}^{\prime}}{\partial x_{j}} \frac{\partial v^{\prime}}{\partial x_{l}}}+\overline{\frac{\partial v^{\prime}}{\partial x_{j}} \frac{\partial u_{l}^{\prime}}{\partial x_{i}} \frac{\partial v^{\prime}}{\partial x_{l}}}+\overline{u_{l}^{\prime} \frac{\partial}{\partial x_{l}}\left(\frac{\partial v^{\prime}}{\partial x_{i}} \frac{\partial v^{\prime}}{\partial x_{j}}\right)}=0
\end{aligned}
$$

We can see there are $Z_{i j}$ terms and various new double and triple correlations. Using the definition of $Z_{i j}$ we get the following transport equation, which describes the evolution of configuration of the nonhomogeneities

$$
\begin{gathered}
\frac{\partial Z_{i j}}{\partial t}+Z_{i l} \frac{\partial \bar{u}_{l}}{\partial x_{j}}+Z_{j l} \frac{\partial \bar{u}_{l}}{\partial x_{i}}+\bar{u}_{l} \frac{\partial Z_{i j}}{\partial x_{l}} \\
+\frac{\partial v^{\prime}}{\partial x_{i}} \frac{\partial u_{l}^{\prime}}{\partial x_{j}} \frac{\partial \bar{v}}{\partial x_{l}}+\frac{\overline{\partial v^{\prime}}}{\partial x_{j}} \frac{\partial u_{l}^{\prime}}{\partial x_{i}} \frac{\partial \bar{v}}{\partial x_{l}}+\overline{u_{l}^{\prime} \frac{\partial v^{\prime}}{\partial x_{i}} \frac{\partial^{2} \bar{v}}{\partial x_{j} \partial x_{l}}+\overline{u_{l}^{\prime} \frac{\partial v^{\prime}}{\partial x_{j}}} \frac{\partial^{2} \bar{v}}{\partial x_{i} \partial x_{l}}} \\
+\overline{\frac{\partial v^{\prime}}{\partial x_{i}} \frac{\partial u_{l}^{\prime}}{\partial x_{j}} \frac{\partial v^{\prime}}{\partial x_{l}}}+\frac{\partial v^{\prime}}{\partial x_{j}} \frac{\partial u_{l}^{\prime}}{\partial x_{i}} \frac{\partial v^{\prime}}{\partial x_{l}}+\frac{\partial}{u_{l}^{\prime} \frac{\partial}{\partial x_{l}}\left(\frac{\partial v^{\prime}}{\partial x_{i}} \frac{\partial v^{\prime}}{\partial x_{j}}\right)}=0
\end{gathered}
$$

This is an exact transport equation of the $Z_{i j}$ tensor and again some moments arise because of the moment used to derive this transport equation. All the second and third order correlations not previously defined need to be modeled or a transport equation needs to be developed for them. 
If we were to develop a transport equation for each triple correlation in equations (4.4), (4.10), (4.15), and (4.21) we would find fourth order correlations in each transport equation. In general, developing a transport equation for an $n^{\text {th }}$ order correlation would introduce $(n+1)^{\text {th }}$ correlations. We do not want to develop an infinite series of transport equations so we "model" or approximate these double and triple correlations.

\subsection{Distribution of Energy (Exact)}

The total energy in the system is contained in three modes, mean internal energy, mean kinetic energy, and turbulent kinetic energy. In section 2.3 the total energy per unit mass was defined as the sum of the internal energy and the total kinetic energy; in unaveraged form we have

$$
E_{\text {total }}=e+\frac{1}{2} u_{i} u_{i}
$$

The total energy can be expressed in terms of the mean internal energy $\bar{e}$, the mean kinetic energy, $K E$, and the turbulent kinetic energy, $K$, by making a Reynolds decomposition and taking the ensemble average of the total energy

$$
E_{\text {total }}=\bar{e}+\frac{1}{2} \bar{u}_{i} \bar{u}_{i}+\frac{1}{2} \overline{u_{i}^{\prime} u_{i}^{\prime}}
$$

Thus the mean kinetic energy is identified as $K E=\frac{1}{2} \bar{u}_{i} \bar{u}_{i}$ and the turbulent kinetic energy is $K=\frac{1}{2} \overline{u_{i}^{\prime} u_{i}^{\prime}}$. Because the Reynolds stress tensor is $R_{i j}=\overline{u_{i}^{\prime} u_{j}^{\prime}}$, then one-half the contraction of $R_{i j}$ is equal to the turbulent kinetic energy, i.e. $K=\frac{1}{2} R_{l l}$. A transport equation for the mean internal energy equation has already been derived, equation (3.7) 


$$
\begin{gathered}
\frac{\partial \bar{e}}{\partial t}+\frac{\partial \bar{e} \bar{u}_{l}}{\partial x_{l}}+\frac{\partial \overline{e^{\prime} u_{l}^{\prime}}}{\partial x_{l}}=\bar{v}\left(\frac{\partial \bar{u}_{i}}{\partial x_{l}}+\frac{\partial \bar{u}_{l}}{\partial x_{i}}\right) \frac{\partial \bar{u}_{i}}{\partial x_{l}}+\bar{v}\left(\frac{\partial u_{i}^{\prime}}{\partial x_{l}} \frac{\partial u_{i}^{\prime}}{\partial x_{l}}+\frac{\partial^{2} R_{l i}}{\partial x_{l} \partial x_{i}}\right) \\
+\left(\frac{\partial N_{i}}{\partial x_{l}}-M_{i l}+\frac{\partial N_{l}}{\partial x_{i}}-M_{l i}\right) \frac{\partial \bar{u}_{i}}{\partial x_{l}}+\left(\frac{\partial N_{i}}{\partial x_{l}}-M_{i l}\right)\left(\frac{\partial \bar{u}_{i}}{\partial x_{l}}+\frac{\partial \bar{u}_{l}}{\partial x_{i}}\right) \\
+\overline{v^{\prime} \frac{\partial u_{i}^{\prime}}{\partial x_{l}} \frac{\partial u_{i}^{\prime}}{\partial x_{l}}}+v^{\prime} \frac{\partial u_{l}^{\prime}}{\partial x_{i}} \frac{\partial u_{i}^{\prime}}{\partial x_{l}}
\end{gathered}
$$

The mean kinetic energy equation can be derived by taking a $\bar{u}_{i}$ moment of the mean momentum equation (3.6)

$$
\begin{gathered}
\bar{u}_{i}\left\{\frac{\partial \bar{u}_{i}}{\partial t}+\frac{\partial \bar{u}_{i} \bar{u}_{l}}{\partial x_{l}}+\frac{\partial R_{i l}}{\partial x_{l}}=-\frac{\partial \bar{p}}{\partial x_{i}}+\left(\frac{\partial \bar{u}_{i}}{\partial x_{l}}+\frac{\partial \bar{u}_{l}}{\partial x_{i}}\right) \frac{\partial \bar{v}}{\partial x_{l}}+\bar{v} \frac{\partial^{2} \bar{u}_{i}}{\partial x_{l}^{2}}\left[\bar{v}\left(\frac{\partial \bar{u}_{i}}{\partial x_{l}}+\frac{\partial \bar{u}_{l}}{\partial x_{i}}\right)\right]\right. \\
\left.+\frac{\partial^{2} N_{i}}{\partial x_{l}^{2}}+\frac{\partial^{2} N_{l}}{\partial x_{l} \partial x_{i}}-\frac{\partial\left(M_{i l}+M_{l i}\right)}{\partial x_{l}}\right\}
\end{gathered}
$$

Rewriting equation (4.23) results in the following

$$
\begin{gathered}
\frac{\partial}{\partial t}\left(\frac{1}{2} \bar{u}_{i} \bar{u}_{i}\right)+\frac{\partial}{\partial x_{l}}\left(\frac{1}{2} \bar{u}_{i} \bar{u}_{i} \bar{u}_{l}\right)+\bar{u}_{i} \frac{\partial R_{i l}}{\partial x_{l}}=-\bar{u}_{i} \frac{\partial \bar{p}}{\partial x_{i}}+\left[\frac{\partial}{\partial x_{l}}\left(\frac{1}{2} \bar{u}_{i} \bar{u}_{i}\right)+\frac{\partial \bar{u}_{i} \bar{u}_{l}}{\partial x_{i}}\right] \frac{\partial \bar{v}}{\partial x_{l}} \\
+\bar{v} \frac{\partial^{2}}{\partial x_{l}^{2}}\left(\frac{1}{2} \bar{u}_{i} \bar{u}_{i}\right)-\bar{v} \frac{\partial \bar{u}_{i}}{\partial x_{l}} \frac{\partial \bar{u}_{i}}{\partial x_{l}}+\bar{u}_{i} \frac{\partial^{2} N_{i}}{\partial x_{l}^{2}}+\bar{u}_{i} \frac{\partial^{2} N_{l}}{\partial x_{l} \partial x_{i}}-\bar{u}_{i} \frac{\partial\left(M_{i l}+M_{l i}\right)}{\partial x_{l}} \\
\frac{\partial K E}{\partial t}+\bar{u}_{l} \frac{\partial K E}{\partial x_{l}}+\bar{u}_{i} \frac{\partial R_{i l}}{\partial x_{l}}=-\bar{u}_{i} \frac{\partial \bar{p}}{\partial x_{i}}+\left[\frac{\partial K E}{\partial x_{l}}+\frac{\partial \bar{u}_{i} \bar{u}_{l}}{\partial x_{i}}\right] \frac{\partial \bar{v}}{\partial x_{l}} \\
+\bar{v} \frac{\partial^{2} K E}{\partial x_{l}^{2}}-\bar{v} \frac{\partial \bar{u}_{i}}{\partial x_{l}} \frac{\partial \bar{u}_{i}}{\partial x_{l}}+\bar{u}_{i} \frac{\partial^{2} N_{i}}{\partial x_{l}^{2}}+\bar{u}_{i} \frac{\partial^{2} N_{l}}{\partial x_{l} \partial x_{i}}-\bar{u}_{i} \frac{\partial\left(M_{i l}+M_{l i}\right)}{\partial x_{l}}
\end{gathered}
$$

The turbulent kinetic energy transport equation can be obtained by contracting the Reynolds stress equation (4.4) 


$$
\begin{gathered}
\frac{\partial R_{k k}}{\partial t}+2 R_{k l} \frac{\partial \bar{u}_{k}}{\partial x_{l}}+\bar{u}_{l} \frac{\partial R_{k k}}{\partial x_{l}}+\frac{\overline{\partial u_{k}^{\prime} u_{k}^{\prime} u_{l}^{\prime}}}{\partial x_{l}}=-\overline{u_{k}^{\prime} \frac{\partial p^{\prime}}{\partial x_{k}}} \\
+2 M_{k l}\left(\frac{\partial \bar{u}_{k}}{\partial x_{l}}+\frac{\partial \bar{u}_{l}}{\partial x_{k}}\right)+2 N_{k} \frac{\partial^{2} \bar{u}_{k}}{\partial x_{l}^{2}}+\left(\frac{\partial R_{k k}}{\partial x_{l}}+2 \overline{\left.u_{k}^{\prime} \frac{\partial u_{l}^{\prime}}{\partial x_{k}}\right)} \frac{\partial \bar{v}}{\partial x_{l}}\right. \\
+\bar{v} \frac{\partial^{2} R_{k k}}{\partial x_{l}^{2}}-2 \bar{v} \frac{\overline{\partial u_{k}^{\prime}}}{\partial x_{l}} \frac{\partial u_{k}^{\prime}}{\partial x_{l}}+2 \overline{u_{k}^{\prime} \frac{\partial}{\partial x_{l}}\left[v^{\prime}\left(\frac{\partial u_{k}^{\prime}}{\partial x_{l}}+\frac{\partial u_{l}^{\prime}}{\partial x_{k}}\right)\right]}
\end{gathered}
$$

Replacing $R_{k k}$ with $2 K$ we get the following

$$
\begin{aligned}
& 2 \frac{\partial K}{\partial t}+2 R_{k l} \frac{\partial \bar{u}_{k}}{\partial x_{l}}+2 \bar{u}_{l} \frac{\partial K}{\partial x_{l}}+\overline{\frac{\partial u_{k}^{\prime} u_{k}^{\prime} u_{l}^{\prime}}{\partial x_{l}}}=-\overline{u_{k}^{\prime} \frac{\partial p^{\prime}}{\partial x_{k}}} \\
& +2 M_{k l}\left(\frac{\partial \bar{u}_{k}}{\partial x_{l}}+\frac{\partial \bar{u}_{l}}{\partial x_{k}}\right)+2 N_{k} \frac{\partial^{2} \bar{u}_{k}}{\partial x_{l}^{2}}+\left(2 \frac{\partial K}{\partial x_{l}}+2 \overline{u_{k}^{\prime} \frac{\partial u_{l}^{\prime}}{\partial x_{k}}}\right) \frac{\partial \bar{v}}{\partial x_{l}} \\
& +2 \bar{v} \frac{\partial^{2} K}{\partial x_{l}^{2}}-2 \bar{v} \overline{\frac{\partial u_{k}^{\prime}}{\partial x_{l}} \frac{\partial u_{k}^{\prime}}{\partial x_{l}}}+2 \overline{u_{k}^{\prime} \frac{\partial}{\partial x_{l}}\left[v^{\prime}\left(\frac{\partial u_{k}^{\prime}}{\partial x_{l}}+\frac{\partial u_{l}^{\prime}}{\partial x_{k}}\right)\right]} \\
& \frac{\partial K}{\partial t}+R_{k l} \frac{\partial \bar{u}_{k}}{\partial x_{l}}+\bar{u}_{l} \frac{\partial K}{\partial x_{l}}+\frac{1}{2} \overline{\frac{\partial u_{k}^{\prime} u_{k}^{\prime} u_{l}^{\prime}}{\partial x_{l}}}=\overline{-u_{k}^{\prime} \frac{\partial p^{\prime}}{\partial x_{k}}} \\
& +M_{k l}\left(\frac{\partial \bar{u}_{k}}{\partial x_{l}}+\frac{\partial \bar{u}_{l}}{\partial x_{k}}\right)+N_{k} \frac{\partial^{2} \bar{u}_{k}}{\partial x_{l}^{2}}+\left(\frac{\partial K}{\partial x_{l}}+\overline{u_{k}^{\prime}} \frac{\partial u_{l}^{\prime}}{\partial x_{k}}\right) \frac{\partial \bar{v}}{\partial x_{l}} \\
& +\bar{v} \frac{\partial^{2} K}{\partial x_{l}^{2}}-\bar{v} \overline{\frac{\partial u_{k}^{\prime}}{\partial x_{l}} \frac{\partial u_{k}^{\prime}}{\partial x_{l}}}+\overline{u_{k}^{\prime} \frac{\partial}{\partial x_{l}}\left[v^{\prime}\left(\frac{\partial u_{k}^{\prime}}{\partial x_{l}}+\frac{\partial u_{l}^{\prime}}{\partial x_{k}}\right)\right]}
\end{aligned}
$$




\subsection{Summary of Exact Transport Equations}

Incompressibility condition equation (3.5)

$$
\frac{\partial \bar{u}_{l}}{\partial x_{l}}=0 \quad \& \quad \frac{\partial u_{l}^{\prime}}{\partial x_{l}}=0
$$

Mean momentum equation (3.6)

$$
\begin{gathered}
\frac{\partial \bar{u}_{i}}{\partial t}+\frac{\partial \bar{u}_{u_{i}} \bar{u}_{l}}{\partial x_{l}}+\frac{\partial R_{i l}}{\partial x_{l}}=-\frac{\partial \bar{p}}{\partial x_{i}}+\frac{\partial}{\partial x_{l}}\left[\bar{v}\left(\frac{\partial \bar{u}_{i}}{\partial x_{l}}+\frac{\partial \bar{u}_{l}}{\partial x_{i}}\right)\right] \\
+\frac{\partial}{\partial x_{l}}\left[\frac{\partial N_{i}}{\partial x_{l}}-M_{i l}+\frac{\partial N_{l}}{\partial x_{i}}-M_{l i}\right]
\end{gathered}
$$

Mean internal energy equation (3.7)

$$
\begin{aligned}
& \frac{\partial \bar{e}}{\partial t}+\frac{\partial \bar{e} \bar{u}_{l}}{\partial x_{l}}+\frac{\partial \overline{e^{\prime} u_{l}^{\prime}}}{\partial x_{l}}=\bar{v}\left(\frac{\partial \bar{u}_{i}}{\partial x_{l}}+\frac{\partial \bar{u}_{l}}{\partial x_{i}}\right) \frac{\partial \bar{u}_{i}}{\partial x_{l}}+\bar{v}\left(\overline{\frac{\partial u_{i}^{\prime}}{\partial x_{l}} \frac{\partial u_{i}^{\prime}}{\partial x_{l}}}+\frac{\partial^{2} R_{l i}}{\partial x_{l} \partial x_{i}}\right) \\
& +\left(\frac{\partial N_{i}}{\partial x_{l}}-M_{i l}+\frac{\partial N_{l}}{\partial x_{i}}-M_{l i}\right) \frac{\partial \bar{u}_{i}}{\partial x_{l}}+\left(\frac{\partial N_{i}}{\partial x_{l}}-M_{i l}\right)\left(\frac{\partial \bar{u}_{i}}{\partial x_{l}}+\frac{\partial \bar{u}_{l}}{\partial x_{i}}\right) \\
& +\overline{v^{\prime} \frac{\partial u_{i}^{\prime}}{\partial x_{l}} \frac{\partial u_{i}^{\prime}}{\partial x_{l}}}+\overline{v^{\prime} \frac{\partial u_{l}^{\prime}}{\partial x_{i}} \frac{\partial u_{i}^{\prime}}{\partial x_{l}}}
\end{aligned}
$$

Evolution of the mean kinematic viscosity (3.8)

$$
\frac{\partial \bar{v}}{\partial t}+\bar{u}_{l} \frac{\partial \bar{v}}{\partial x_{l}}+M_{l l}=0 \quad \text { or } \quad \frac{\partial \bar{v}}{\partial t}+\bar{u}_{l} \frac{\partial \bar{v}}{\partial x_{l}}+\frac{\partial N_{l}}{\partial x_{l}}=0
$$


Reynolds stress transport equation (4.4)

$$
\begin{aligned}
\frac{\partial R_{i j}}{\partial t}+ & R_{j l} \frac{\partial \bar{u}_{i}}{\partial x_{l}}+R_{i l} \frac{\partial \bar{u}_{j}}{\partial x_{l}}+\bar{u}_{l} \frac{\partial R_{i j}}{\partial x_{l}}+\frac{\overline{\partial u_{i}^{\prime} u_{j}^{\prime} u_{l}^{\prime}}}{\partial x_{l}}=\overline{\overline{u_{j}^{\prime}} \frac{\partial p^{\prime}}{\partial x_{i}}}-\overline{u_{i}^{\prime} \frac{\partial p^{\prime}}{\partial x_{j}}} \\
+ & M_{j l}\left(\frac{\partial \bar{u}_{i}}{\partial x_{l}}+\frac{\partial \bar{u}_{l}}{\partial x_{i}}\right)+N_{j} \frac{\partial^{2} \bar{u}_{i}}{\partial x_{l}^{2}}+M_{i l}\left(\frac{\partial \bar{u}_{j}}{\partial x_{l}}+\frac{\partial \bar{u}_{l}}{\partial x_{j}}\right)+N_{i} \frac{\partial^{2} \bar{u}_{j}}{\partial x_{l}^{2}} \\
+ & \left(\frac{\partial R_{i j}}{\partial x_{l}}+\overline{\left.u_{j}^{\prime} \frac{\partial u_{l}^{\prime}}{\partial x_{i}}+\overline{u_{i}^{\prime} \frac{\partial u_{l}^{\prime}}{\partial x_{j}}}\right) \frac{\partial \bar{v}}{\partial x_{l}}+\bar{v} \frac{\partial^{2} R_{i j}}{\partial x_{l}^{2}}-2 \bar{v} \frac{\partial u_{i}^{\prime}}{\partial x_{l}} \frac{\partial u_{j}^{\prime}}{\partial x_{l}}}\right. \\
& +u_{j}^{\prime} \frac{\partial}{\partial x_{l}}\left[v^{\prime}\left(\frac{\partial u_{i}^{\prime}}{\partial x_{l}}+\frac{\partial u_{l}^{\prime}}{\partial x_{i}}\right)\right]+u_{i}^{\prime} \frac{\partial}{\partial x_{l}}\left[v^{\prime}\left(\frac{\partial u_{j}^{\prime}}{\partial x_{l}}+\frac{\partial u_{l}^{\prime}}{\partial x_{j}}\right)\right]
\end{aligned}
$$

$M_{i j}$ transport equation (4.10)

$$
\begin{aligned}
& \frac{\partial M_{i j}}{\partial t}+M_{l j} \frac{\partial \bar{u}_{i}}{\partial x_{l}}+\overline{u_{i}^{\prime} \frac{\partial u_{l}^{\prime}}{\partial x_{j}}} \frac{\partial \bar{v}}{\partial x_{l}}+R_{i l} \frac{\partial^{2} \bar{v}}{\partial x_{l}^{2}}+M_{i l} \frac{\partial \bar{u}_{l}}{\partial x_{j}}+\bar{u}_{l} \frac{\partial M_{i j}}{\partial x_{l}} \\
& +\overline{u_{l}^{\prime} \frac{\partial v^{\prime}}{\partial x_{j}} \frac{\partial u_{i}^{\prime}}{\partial x_{l}}}+\overline{u_{i}^{\prime} \frac{\partial}{\partial x_{j}}\left(u_{l}^{\prime} \frac{\partial v^{\prime}}{\partial x_{l}}\right)}=-\overline{\frac{\partial v^{\prime}}{\partial x_{j}} \frac{\partial p^{\prime}}{\partial x_{i}}}+Z_{j k}\left(\frac{\partial \bar{u}_{i}}{\partial x_{k}}+\frac{\partial \bar{u}_{k}}{\partial x_{i}}\right) \\
& +\frac{1}{2} \frac{\partial \overline{v^{\prime} v^{\prime}}}{\partial x_{j}} \frac{\partial^{2} \bar{u}_{i}}{\partial x_{k}^{2}}+\left(\overline{\frac{\partial v^{\prime}}{\partial x_{j}} \frac{\partial u_{i}^{\prime}}{\partial x_{k}}}+\frac{\overline{\partial v^{\prime}}}{\partial x_{j}} \frac{\partial u_{k}^{\prime}}{\partial x_{i}}\right) \frac{\partial \bar{v}}{\partial x_{k}}+\bar{v} \frac{\partial^{2} M_{i j}}{\partial x_{l}^{2}}-2 \bar{v} \frac{\partial u_{i}^{\prime}}{\partial x_{l}} \frac{\partial^{2} v^{\prime}}{\partial x_{l} \partial x_{j}} \\
& -\bar{v} \overline{u_{i}^{\prime} \frac{\partial^{3} v^{\prime}}{\partial x_{l}^{2} \partial x_{j}}}+\overline{\frac{\partial v^{\prime}}{\partial x_{j}} \frac{\partial v^{\prime}}{\partial x_{k}}\left(\frac{\partial u_{i}^{\prime}}{\partial x_{k}}+\frac{\partial u_{k}^{\prime}}{\partial x_{i}}\right)}+\overline{v^{\prime} \frac{\partial v^{\prime}}{\partial x_{j}} \frac{\partial^{2} u_{i}^{\prime}}{\partial x_{k}^{2}}}
\end{aligned}
$$

$N_{i}$ transport equation (4.15)

$$
\begin{gathered}
\frac{\partial N_{i}}{\partial t}+N_{l} \frac{\partial \bar{u}_{i}}{\partial x_{l}}+\bar{u}_{l} \frac{\partial N_{i}}{\partial x_{l}}+R_{i l} \frac{\partial \bar{v}}{\partial x_{l}}+\frac{\partial \overline{u_{i}^{\prime} v^{\prime} u_{l}^{\prime}}}{\partial x_{l}}=-\overline{v^{\prime} \frac{\partial p^{\prime}}{\partial x_{i}}} \\
+\frac{1}{2} \frac{\partial \overline{v^{\prime} v^{\prime}}}{\partial x_{k}}\left(\frac{\partial \bar{u}_{i}}{\partial x_{k}}+\frac{\partial \bar{u}_{k}}{\partial x_{i}}\right)+\overline{v^{\prime} v^{\prime}} \frac{\partial^{2} \bar{u}_{i}}{\partial x_{k}^{2}}+\left(\frac{\partial N_{i}}{\partial x_{k}}-M_{i k}+\frac{\partial N_{k}}{\partial x_{i}}-M_{k i}\right) \frac{\partial \bar{v}}{\partial x_{k}} \\
+\bar{v} \frac{\partial^{2} N_{i}}{\partial x_{k}^{2}}-\bar{v} \frac{\partial M_{i k}}{\partial x_{k}}-\bar{v} \frac{\partial v^{\prime}}{\partial x_{k}} \frac{\partial u_{i}^{\prime}}{\partial x_{k}}+v^{\prime} \frac{\partial}{\partial x_{k}}\left[v^{\prime}\left(\frac{\partial u_{i}^{\prime}}{\partial x_{k}}+\frac{\partial u_{k}^{\prime}}{\partial x_{i}}\right)\right]
\end{gathered}
$$


$Z_{i j}$ transport equation (4.21)

$$
\begin{aligned}
& \frac{\partial Z_{i j}}{\partial t}+Z_{i l} \frac{\partial \bar{u}_{l}}{\partial x_{j}}+Z_{j l} \frac{\partial \bar{u}_{l}}{\partial x_{i}}+\bar{u}_{l} \frac{\partial Z_{i j}}{\partial x_{l}} \\
& +\overline{\frac{\partial v^{\prime}}{\partial x_{i}} \frac{\partial u_{l}^{\prime}}{\partial x_{j}}} \frac{\partial \bar{v}}{\partial x_{l}}+\overline{\frac{\partial v^{\prime}}{\partial x_{j}} \frac{\partial u_{l}^{\prime}}{\partial x_{i}}} \frac{\partial \bar{v}}{\partial x_{l}}+\overline{u_{l}^{\prime} \frac{\partial v^{\prime}}{\partial x_{i}}} \frac{\partial^{2} \bar{v}}{\partial x_{j} \partial x_{l}}+\overline{u_{l}^{\prime} \frac{\partial v^{\prime}}{\partial x_{j}}} \frac{\partial^{2} \bar{v}}{\partial x_{i} \partial x_{l}} \\
& +\overline{\frac{\partial v^{\prime}}{\partial x_{i}} \frac{\partial u_{l}^{\prime}}{\partial x_{j}} \frac{\partial v^{\prime}}{\partial x_{l}}}+\overline{\frac{\partial v^{\prime}}{\partial x_{j}} \frac{\partial u_{l}^{\prime}}{\partial x_{i}} \frac{\partial v^{\prime}}{\partial x_{l}}}+\overline{u_{l}^{\prime} \frac{\partial}{\partial x_{l}}\left(\frac{\partial v^{\prime}}{\partial x_{i}} \frac{\partial v^{\prime}}{\partial x_{j}}\right)}=0
\end{aligned}
$$

Mean kinetic energy equation (4.24)

$$
\begin{gathered}
\frac{\partial K E}{\partial t}+\bar{u}_{l} \frac{\partial K E}{\partial x_{l}}+\bar{u}_{i} \frac{\partial R_{i l}}{\partial x_{l}}=-\bar{u}_{i} \frac{\partial \bar{p}}{\partial x_{i}}+\left[\frac{\partial K E}{\partial x_{l}}+\frac{\partial \bar{u}_{i} \bar{u}_{l}}{\partial x_{i}}\right] \frac{\partial \bar{v}}{\partial x_{l}} \\
+\bar{v} \frac{\partial^{2} K E}{\partial x_{l}^{2}}-\bar{v} \frac{\partial \bar{u}_{i}}{\partial x_{l}} \frac{\partial \bar{u}_{i}}{\partial x_{l}}+\bar{u}_{i} \frac{\partial^{2} N_{i}}{\partial x_{l}^{2}}+\bar{u}_{i} \frac{\partial^{2} N_{l}}{\partial x_{l} \partial x_{i}}-\bar{u}_{i} \frac{\partial\left(M_{i l}+M_{l i}\right)}{\partial x_{l}}
\end{gathered}
$$

Turbulent kinetic energy equation (4.25)

$$
\begin{gathered}
\frac{\partial K}{\partial t}+R_{k l} \frac{\partial \bar{u}_{k}}{\partial x_{l}}+\bar{u}_{l} \frac{\partial K}{\partial x_{l}}+\frac{1}{2} \frac{\overline{\partial u_{k}^{\prime} u_{k}^{\prime} u_{l}^{\prime}}}{\partial x_{l}}=\overline{-\overline{u_{k}^{\prime}} \frac{\partial p^{\prime}}{\partial x_{k}}} \\
+M_{k l}\left(\frac{\partial \bar{u}_{k}}{\partial x_{l}}+\frac{\partial \bar{u}_{l}}{\partial x_{k}}\right)+N_{k} \frac{\partial^{2} \bar{u}_{k}}{\partial x_{l}^{2}}+\left(\frac{\partial K}{\partial x_{l}}+\overline{\left.u_{k}^{\prime} \frac{\partial u_{l}^{\prime}}{\partial x_{k}}\right)} \frac{\partial \bar{v}}{\partial x_{l}}\right. \\
+\bar{v} \frac{\partial^{2} K}{\partial x_{l}^{2}}-\bar{v} \overline{\frac{\partial u_{k}^{\prime}}{\partial x_{l}} \frac{\partial u_{k}^{\prime}}{\partial x_{l}}}+\overline{u_{k}^{\prime} \frac{\partial}{\partial x_{l}}\left[v^{\prime}\left(\frac{\partial u_{k}^{\prime}}{\partial x_{l}}+\frac{\partial u_{l}^{\prime}}{\partial x_{k}}\right)\right]}
\end{gathered}
$$

Up to this point we have derived a set of exact transport equations that govern a crenulative circumstance. Even though these equations are exact they do not display closure because of the double and triple correlations; i.e. there are more unknowns than equations. For these equations to become a closed set we must model the double and triple correlations, thus giving us a set of approximate but closed equations. 


\section{Chapter 5}

\section{Modeled Transport Equations in Cartesian Tensor Form}

In this chapter the guidelines for turbulence modeling and a postulated set of circumstances for crenulation are discussed. Also the modeled (closed) transport equations for the Reynolds stress tensor $R_{i j}$, the $M_{i j}$ tensor, and the $Z_{i j}$ tensor are developed. The contraction of $M_{i j}$ and its effects on $N_{i}$ are discussed in some detail. The modeling of the mean internal energy equation and the turbulent kinetic energy equation must be consistent with modeling the transport equation of the Reynolds stress tensor. When developing a closed set of equations the terms in each of the equations must be functions of quantities that can be explicitly determined. This means the number of equations is equal to the number of unknowns. The explicit variables used for modeling are any of the mean quantities, and also the moments $R_{i j}, M_{i j}, N_{i}$, and $Z_{i j}$, and gradients of these quantities.

\subsection{Turbulence Modeling Guidelines}

The main goal of turbulence transport theory is to solve turbulent flow problems without completely solving the Navier-Stokes equations for each fluctuation of the fluid variables. This goal is directly analogous to that of solving the gas dynamics equations instead of the equations for the motion of every molecule. With a turbulence transport model each fluctuation does not have to be resolved, instead an average value of the correlations associated with the turbulence can be calculated. In the development of the turbulence transport models all of the representations must appear in a closed form. Closed form means all the terms in the transport equations are functions of explicitly determined values. This means there will not be more unknowns than equations, as is the case in chapter 4. Also, in the development of a turbulence transport equation it is our goal to try 
to represent as wide a range of turbulent flow circumstances as possible with a single representation [10]. The modeling should therefore be independent of the initial and boundary conditions. In addition, the model must have the capability to display various physical processes such as convection, diffusion, creation and destruction, and exchange of components among the nonisotropic directions of the turbulence quantities. Generally, in the development of turbulence models there is a set of guidelines that must be followed to ensure proper formulation of the transport equations.

1. Proper dimensionality must be maintained. The modeled term must have the same dimensionality as the unmodeled term. The transport equations for $R_{i j}, M_{i j}$, and $Z_{i j}$ all have dimensions of length squared divided by time cubed. This means all modeled terms in those equations must have the dimensions of length squared divided by time cubed.

2. Proper contraction of a tensor must be preserved. The modeled term must contract in the same manner as the unmodeled term. If an unmodeled term contracts to zero so must the modeled term.

3. The conservation properties of the unmodeled term must be preserved. If the unmodeled term is conservative then the modeled term must be conservative as well.

4. Tensor invariance and symmetry must be preserved. The modeled term must exhibit the same tensor properties and index symmetries as the unmodeled term.

5. A model term must exhibit Galilean invariance. This means the modeled term retains the same properties in any inertial frame.

In addition to the modeling guidelines are the constraints of realizability. Any diagonal component of the Reynolds stress tenor must be positive definite, i.e. $R_{11} \geq 0, R_{22} \geq 0$, $R_{33} \geq 0$. The Schwartz inequality applied to the Reynolds stress is $R_{(i i)} R_{(j j)}-R_{(i j)}^{2} \geq 0$, 
where the parentheses indicate the summation is suspended on the indices. In addition there is a constraint among the tensors, $R_{i j}, M_{i j}$, and $Z_{i j}$, which is $Z_{(i i)} R_{(j j)}-M_{(i j)}^{2} \geq 0$, where the brackets indicate the summation is suspended. These constraints must apply everywhere in the domain at all times. It has been proposed, but not yet validated, that the realizability constraints are connected to proper informational entropy behavior.

The modeling of various moments to close the equations often looks more like art than science. We follow the modeling techniques from classical turbulence where possible and postulate some new types of closures where required. Only after considerable testing and modification of the closures will the theory of turbulent crenulation gain the respectability that is only gradually being given to the transport theories for simpler circumstances.

\subsection{An Illustrative Set of Circumstances for Crenulation}

In the past, the study of turbulence transport theory has taking some creativity on the part of the researchers to develop workable models. In the early days various models for classical turbulence were postulated, which at the time were advanced works in the field; but now with the help of high speed computers better models are being introduced. Crenulation is an area of turbulence that has not been investigated extensively, so there are many concepts that are relatively new. Rather than trying to develop a complete model for all possible circumstances, we have worked with a restricted set, which alleviates some of the mathematical complexities and lays the foundation for understanding some of the new concepts of crenulation turbulence.

The first restriction is that the mean kinematic viscosity is constant through space and time. This has a profound effect on the evolution of $N_{i}$, on the contraction of $M_{i j}$ and on the modeling of the transport equation of $M_{i j}$. The second restriction is that the divergence of $\overline{e^{\prime} u_{l}^{\prime}}$ in the transport equation of the mean internal energy is negligible. The 
third restriction is the scalar $\overline{v^{\prime} v^{\prime}}$ in the transport equation for $M_{i j}$ is constant through space and time.

To see the motivation and interpretation of the first restriction, consider the evolution of the mean kinematic viscosity equation (3.8)

$$
\frac{\partial \bar{v}}{\partial t}+\bar{u}_{l} \frac{\partial \bar{v}}{\partial x_{l}}+M_{l l}=0 \quad \text { or } \quad \frac{\partial \bar{v}}{\partial t}+\bar{u}_{l} \frac{\partial \bar{v}}{\partial x_{l}}+\frac{\partial N_{l}}{\partial x_{l}}=0
$$

If the mean kinematic viscosity is constant through space and time then the contraction of $M_{i j}$ is zero and $N_{i}$ is solenoidal, i.e. divergence free

$$
M_{l l}=\frac{\partial N_{l}}{\partial x_{l}}=0
$$

The vanishing contraction of $M_{i j}$ means the transport equation for this variable must contract to zero. In the modeling of the transport equation for $M_{i j} ; M_{l l}=0$ will be taken into consideration. For the restricted set of circumstances one possible choice for $N_{i}$ is to let it be a constant; this choice still satisfies the condition of being solenoidal. The transport equation for the Reynolds stress tensor is thereby simplified and we do not have to consider closures for the evolution equation for $N_{i}$.

The divergence of $\overline{e^{\prime} u_{l}^{\prime}}$ is a term that behaves like the diffusion of the mean internal energy due to the crenulative turbulence. The processes of advection, dissipation to heat, and the other processes occurring in the mean internal energy equation might very likely be the dominant processes, so it could be argued the diffusion of the mean internal energy is negligible For no other reason we have neglected the divergence of $\overline{e^{\prime} u_{l}^{\prime}}$, therefore we do not have to develop a closure for the vector $\overline{e^{\prime} u_{l}^{\prime}}$.

The third restriction is that the scalar, $\overline{v^{\prime} v^{\prime}}$, in the transport equation for $M_{i j}$ is constant through space and time. We can show the reasonableness of this restriction by 
developing a transport equation for $\overline{v^{\prime} v^{\prime}}$. First multiply equation (2.13) by $v^{\prime}$, then make a Reynolds decomposition, and finally take the ensemble average

$$
\begin{gathered}
\overline{v^{\prime}\left[\frac{\partial\left(\bar{v}+v^{\prime}\right)}{\partial t}+\frac{\partial\left(\bar{v}+v^{\prime}\right)\left(\bar{u}_{l}+u_{l}^{\prime}\right)}{\partial x_{l}}\right]}=0 \\
\frac{1}{2} \frac{\partial \overline{v^{\prime} v^{\prime}}}{\partial t}+\frac{1}{2} \bar{u}_{l} \frac{\partial \overline{v^{\prime} v^{\prime}}}{\partial x_{l}}+\overline{v^{\prime} u_{l}^{\prime}} \frac{\partial \bar{v}}{\partial x_{l}}+\frac{1}{2} \overline{u_{l}^{\prime} \frac{\partial v^{\prime} v^{\prime}}{\partial x_{l}}}=0
\end{gathered}
$$

The first two terms on the left side combine into the Lagrangian derivative, and because the mean kinematic viscosity is constant through space and time we obtain

$$
\frac{D \overline{v^{\prime} v^{\prime}}}{D t}+\overline{u_{l}^{\prime} \frac{\partial v^{\prime} v^{\prime}}{\partial x_{l}}}=0
$$

If we assume the triple correlation $\overline{u_{l}^{\prime} \frac{\partial v^{\prime} v^{\prime}}{\partial x_{l}}}$ vanishes, then $\overline{v^{\prime} v^{\prime}}$ is a constant

$$
\frac{D \overline{v^{\prime} v^{\prime}}}{D t}=0 \quad \Rightarrow \quad \overline{v^{\prime} v^{\prime}}=\text { constant }
$$

The correlation $\overline{v^{\prime} v^{\prime}}$ being a constant further simplifies the Reynolds stress equation and the transport equation for this correlation does not have to be included with the rest of the governing equations. 


\subsection{Reynolds Stress Transport Modeling}

The Reynolds stress transport equation (4.4), within the restricted set of circumstances, reduces to

$$
\begin{gathered}
\frac{\partial R_{i j}}{\partial t}+R_{j l} \frac{\partial \bar{u}_{i}}{\partial x_{l}}+R_{i l} \frac{\partial \bar{u}_{j}}{\partial x_{l}}+\bar{u}_{l} \frac{\partial R_{i j}}{\partial x_{l}}+\frac{\overline{\partial u_{i}^{\prime} u_{j}^{\prime} u_{l}^{\prime}}}{\partial x_{l}}=\overline{\overline{u_{j}^{\prime} \frac{\partial p^{\prime}}{\partial x_{i}}}}-\overline{u_{i}^{\prime} \frac{\partial p^{\prime}}{\partial x_{j}}} \\
+M_{j l}\left(\frac{\partial \bar{u}_{i}}{\partial x_{l}}+\frac{\partial \bar{u}_{l}}{\partial x_{i}}\right)+M_{i l}\left(\frac{\partial \bar{u}_{j}}{\partial x_{l}}+\frac{\partial \bar{u}_{l}}{\partial x_{j}}\right)+\bar{v} \frac{\partial^{2} R_{i j}}{\partial x_{l}^{2}}-2 \bar{v} \frac{\overline{\partial u_{i}^{\prime}}}{\partial x_{l}} \frac{\partial u_{j}^{\prime}}{\partial x_{l}} \\
+u_{j}^{\prime} \frac{\partial}{\partial x_{l}}\left[v^{\prime}\left(\frac{\partial u_{i}^{\prime}}{\partial x_{l}}+\frac{\partial u_{l}^{\prime}}{\partial x_{i}}\right)\right]+u_{i}^{\prime} \frac{\partial}{\partial x_{l}}\left[v^{\prime}\left(\frac{\partial u_{j}^{\prime}}{\partial x_{l}}+\frac{\partial u_{l}^{\prime}}{\partial x_{j}}\right)\right]
\end{gathered}
$$

The terms that need no modeling are:

Inertial term

$$
\frac{\partial R_{i j}}{\partial t}
$$

Mean flow coupling terms

$$
R_{j l} \frac{\partial \bar{u}_{i}}{\partial x_{l}} \& R_{i l} \frac{\partial \bar{u}_{j}}{\partial x_{l}}
$$

Advection term

$$
\bar{u}_{l} \frac{\partial R_{i j}}{\partial x_{l}}
$$

$M_{i j}$ coupling to

the mean flow gradient $M_{j l}\left(\frac{\partial \bar{u}_{i}}{\partial x_{l}}+\frac{\partial \bar{u}_{l}}{\partial x_{i}}\right) \& M_{i l}\left(\frac{\partial \bar{u}_{j}}{\partial x_{l}}+\frac{\partial \bar{u}_{l}}{\partial x_{j}}\right)$

Diffusion of the Reynolds stress

due to the mean viscosity

$$
\bar{v} \frac{\partial^{2} R_{i j}}{\partial x_{l}^{2}}
$$


The remaining terms, the triple velocity correlation, the pressure-velocity correlations, and the various other correlations are all terms that need to be modeled. The modeling of these terms follows the above guidelines; some of the terms have been discussed by other researchers. The new terms that arise due to kinematic viscosity fluctuations are modeled using some of the modeling techniques used by other researchers.

The gradient of the triple velocity correlation $\frac{\partial \overline{u_{i}^{\prime} u_{j}^{\prime} u_{l}^{\prime}}}{\partial x_{l}}$ acts as a diffuser of the turbulence, having a tendency to spread the turbulent energy spatially. Daly and Harlow proposed a gradient flux approximation for a triple correlation involving a velocity fluctuation [11]

$$
\overline{A^{\prime} u_{l}^{\prime}} \cong-C \frac{s}{\sqrt{K}} R_{l m} \frac{\partial \bar{A}}{\partial x_{m}}
$$

in which $A$ can be a tensor of any order and can involve the product of two coupled fluctuations; $C$ is some dimensionless parameter, $s$ is a physical length scale associated with the turbulence, $K$ is the turbulent kinetic energy, and $\bar{A}$ is the ensemble average of A. The physical length scale can be associated with the size of the nonhomogeneities, or the separation distance between nonhomogeneities, or the dominant scale of the turbulence. In classical turbulence or variable density turbulence $s$ relates to the dominant turbulent scale. Here we tentatively assume $s$ relates to the scale of nonhomogeneities. Using this model for the triple velocity correlation results in the following model

$$
\frac{\partial \overline{\left(u_{i}^{\prime} u_{j}^{\prime}\right) u_{l}^{\prime}}}{\partial x_{l}}=\frac{\partial \overline{\left(R_{i j}^{\prime}\right) u_{l}^{\prime}}}{\partial x_{l}} \cong \frac{\partial}{\partial x_{l}}\left[-C_{1} \frac{s}{\sqrt{K}} R_{l m} \frac{\partial R_{i j}}{\partial x_{m}}\right]
$$

This term violates the constraint of tensor symmetry explained in the guidelines, but for this model we have relaxed this constraint without destroying the effect of the triple correlation. 
We relaxed the tensor symmetry guideline purely as a first-cut simplification, in which the flux of a Reynolds stress component depends only on the gradient of that component.

We can rewrite the pressure-velocity correlations in conservative and vanishing contraction parts

$$
\overline{-u_{j}^{\prime} \frac{\partial p^{\prime}}{\partial x_{i}}}-\overline{u_{i}^{\prime} \frac{\partial p^{\prime}}{\partial x_{j}}}=-\left(\overline{\frac{\partial u_{j}^{\prime} p^{\prime}}{\partial x_{i}}}+\overline{\frac{\partial u_{i}^{\prime} p^{\prime}}{\partial x_{j}}}\right)+\overline{p^{\prime}\left(\frac{\partial u_{j}^{\prime}}{\partial x_{i}}+\frac{\partial u_{i}^{\prime}}{\partial x_{j}}\right)}
$$

The term $-\left(\overline{\frac{\partial u_{j}^{\prime} p^{\prime}}{\partial x_{i}}}+\frac{\overline{\partial u_{i}^{\prime} p^{\prime}}}{\partial x_{j}}\right)$ is conservative because it is the gradient of a flux. Daly and Harlow [11] proposed the simple diffusion approximation for the conservative part of the pressure-velocity correlation

$$
-\left(\overline{\frac{\partial u_{j}^{\prime} p^{\prime}}{\partial x_{i}}}+\frac{\overline{\partial u_{i}^{\prime} p^{\prime}}}{\partial x_{j}}\right) \cong \frac{\partial}{\partial x_{l}}\left[C_{2} \frac{s}{\sqrt{K}} R_{l m} \frac{\partial R_{i j}}{\partial x_{m}}\right]
$$

The model for the gradient of the triple velocity correlation, equation (5.1), and the model for the conservative part of the pressure-velocity correlation, equation (5.2), can be combined into one term

$$
-\frac{\overline{\partial u_{i}^{\prime} u_{j}^{\prime} u_{l}^{\prime}}}{\partial x_{l}}-\left(\overline{\frac{\partial u_{j}^{\prime} p^{\prime}}{\partial x_{i}}}+\frac{\overline{\partial u_{i}^{\prime} p^{\prime}}}{\partial x_{j}}\right) \cong \frac{\partial}{\partial x_{l}}\left[C_{D} \frac{s}{\sqrt{K}} R_{l m} \frac{\partial R_{i j}}{\partial x_{m}}\right]
$$

where $C_{D}=C_{1}+C_{2}$ is a dimensionless parameter. This model represents the turbulent self diffusion.

The term $\overline{p^{\prime}\left(\frac{\partial u_{j}^{\prime}}{\partial x_{i}}+\frac{\partial u_{i}^{\prime}}{\partial x_{j}}\right)}$ has vanishing contraction and is nonconservative. This term does not contribute to the turbulent kinetic energy in any location because of the vanishing contraction, but may exchange energy among the diagonal components of the 
Reynolds stress tensor. Launder, Reece, and Rodi [12] proposed a "rapid pressure term" and Rotta [13] proposed the addition of a "return to isotropy term"

$$
\begin{aligned}
\overline{p^{\prime}\left(\frac{\partial u_{j}^{\prime}}{\partial x_{i}}+\frac{\partial u_{i}^{\prime}}{\partial x_{j}}\right)} & \cong C_{B}\left(R_{i l} \frac{\partial \bar{u}_{j}}{\partial x_{l}}+R_{j l} \frac{\partial \bar{u}_{i}}{\partial x_{l}}-\frac{2}{3} R_{k l} \frac{\partial \bar{u}_{k}}{\partial x_{l}} \delta_{i j}\right) \\
& +C_{R} \frac{\sqrt{K}}{s}\left(\frac{2}{3} K \delta_{i j}-R_{i j}\right)
\end{aligned}
$$

where $C_{B}$ and $C_{R}$ are dimensionless parameters. We assume that the return to isotropy is enhanced by collisions of the nonhomogeneities, which are driven by the mean flow contortions in the system. The nonhomogeneities can interact with one another because the mean flow contortions bring them together. This gives the opportunity for stretching, coalescence, and fragmentation of the nonhomogeneities in the system, although in this thesis we have ignored the possibility of coalescence or fragmentation. Through this additional collision term we can enhance the rate at which the diagonal components of the Reynolds stress tensor become isotropic. The modified model is represented by the addition of the $C_{H R}$ term

$$
\begin{gathered}
\overline{p^{\prime}\left(\frac{\partial u_{j}^{\prime}}{\partial x_{i}}+\frac{\partial u_{i}^{\prime}}{\partial x_{j}}\right)} \equiv C_{B}\left(R_{i l} \frac{\partial \bar{u}_{j}}{\partial x_{l}}+R_{j l} \frac{\partial \bar{u}_{i}}{\partial x_{l}}-\frac{2}{3} R_{k l} \frac{\partial \bar{u}_{k}}{\partial x_{l}} \delta_{i j}\right) \\
+\left(C_{R} \frac{\sqrt{K}}{s}+C_{H R} \sqrt{\left.\frac{\partial \bar{u}_{k}}{\partial x_{l}} \frac{\partial \bar{u}_{k}}{\partial x_{l}}\right)}\right)\left(\frac{2}{3} K \delta_{i j}-R_{i j}\right)
\end{gathered}
$$

where $C_{H R}$ is a dimensionless parameter. This model describes both the redistribution of Reynolds stress components and the return to isotropy of the turbulence.

The correlation $-2 \bar{v} \frac{\overline{\partial u_{i}^{\prime}}}{\partial x_{l}} \frac{\partial u_{j}^{\prime}}{\partial x_{l}}$, dissipates the Reynolds stress components (and thus the turbulence energy) due to the mean kinematic viscosity. In classical turbulence this term is usually modeled by a dissipation term $\varepsilon_{i j}$ yielding a $K-\varepsilon$ turbulence model, but 
the introduction of an epsilon term implies the turbulence energy is being dissipated to heat at the finest scales; whereas, in reality, the dissipation refers to cascade of Reynolds stress components to fine scales. In our viscous crenulation the turbulence is dissipating to heat at scales on the order $s$, the size of the nonhomogeneities. Daly and Harlow [11] proposed the general approximation

$$
\overline{\frac{\partial A^{\prime}}{\partial x_{l}} \frac{\partial B^{\prime}}{\partial x_{l}}} \cong C \frac{\overline{A^{\prime} B^{\prime}}}{s^{2}}
$$

where $A^{\prime}$ and $B^{\prime}$ can be any fluctuational quantities. Applying this closure recipe to the viscosity term we get the following equation

$$
-2 \overline{\frac{\partial u_{i}^{\prime}}{\partial x_{l}} \frac{\partial u_{j}^{\prime}}{\partial x_{l}}} \cong-2 \bar{v} \frac{\Delta \overline{u_{i}^{\prime} u_{j}^{\prime}}}{s^{2}}
$$

where $\Delta$ is a function of the turbulent Reynolds number and $s$ is the same physical length scale used in the model for the triple velocity correlation.

$$
-2 \bar{v} \frac{\overline{\partial u_{i}^{\prime}}}{\partial x_{l} \frac{\partial u_{j}^{\prime}}{\partial x_{l}}} \cong-2 \bar{v} \frac{\Delta R_{i j}}{s^{2}}
$$

The models for the triple correlations in equation (4.4) involving a viscosity fluctuation, $\overline{u_{j}^{\prime} \frac{\partial}{\partial x_{l}}\left[v^{\prime}\left(\frac{\partial u_{i}^{\prime}}{\partial x_{l}}+\frac{\partial u_{l}^{\prime}}{\partial x_{i}}\right)\right]}$ and $\overline{u_{i}^{\prime} \frac{\partial}{\partial x_{l}}\left[v^{\prime}\left(\frac{\partial u_{j}^{\prime}}{\partial x_{l}}+\frac{\partial u_{l}^{\prime}}{\partial x_{j}}\right)\right]}$, have similar forms as the mean viscous diffusion $\bar{v} \frac{\partial^{2} R_{i j}}{\partial x_{l}^{2}}$ and the unmodeled decay term $-2 \bar{v} \frac{\partial u_{i}^{\prime}}{\partial x_{l}} \frac{\partial u_{j}^{\prime}}{\partial x_{l}}$ in an indirect way. The decay term is large compared to the mean viscous diffusion, and since the form of the triple correlations is similar to these terms we added an enhancement to the decay term through the parameter $Q$ to model these triple correlations 


$$
\overline{u_{j}^{\prime} \frac{\partial}{\partial x_{l}}\left[v^{\prime}\left(\frac{\partial u_{i}^{\prime}}{\partial x_{l}}+\frac{\partial u_{l}^{\prime}}{\partial x_{i}}\right)\right]}+\overline{u_{i}^{\prime} \frac{\partial}{\partial x_{l}}\left[v^{\prime}\left(\frac{\partial u_{j}^{\prime}}{\partial x_{l}}+\frac{\partial u_{l}^{\prime}}{\partial x_{j}}\right)\right]} \cong-Q \frac{\Delta R_{i j}}{s^{2}}
$$

The result of combining the two models

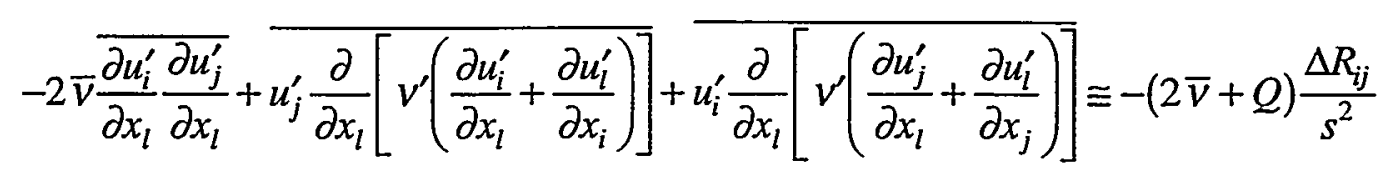

Substituting the modeled terms for the exact terms results in a modeled, but closed Reynolds stress tensor equation

$$
\begin{gathered}
\frac{\partial R_{i j}}{\partial t}+R_{j l} \frac{\partial \bar{u}_{i}}{\partial x_{l}}+R_{i l} \frac{\partial \bar{u}_{j}}{\partial x_{l}}+\bar{u}_{l} \frac{\partial R_{i j}}{\partial x_{l}}=\frac{\partial}{\partial x_{l}}\left[C_{D} \frac{s}{\sqrt{K}} R_{l m} \frac{\partial R_{i j}}{\partial x_{m}}\right] \\
+C_{B}\left(R_{i l} \frac{\partial \bar{u}_{j}}{\partial x_{l}}+R_{j l} \frac{\partial \bar{u}_{i}}{\partial x_{l}}-\frac{2}{3} R_{k l} \frac{\partial \bar{u}_{k}}{\partial x_{l}} \delta_{i j}\right)+\left(C_{R} \frac{\sqrt{K}}{s}+C_{H R} \sqrt{\frac{\partial \bar{u}_{k}}{\partial x_{l}} \frac{\partial \bar{u}_{k}}{\partial x_{l}}}\right)\left(\frac{2}{3} K \delta_{i j}-R_{i j}\right) \\
+M_{j l}\left(\frac{\partial \bar{u}_{i}}{\partial x_{l}}+\frac{\partial \bar{u}_{l}}{\partial x_{i}}\right)+M_{i l}\left(\frac{\partial \bar{u}_{j}}{\partial x_{l}}+\frac{\partial \bar{u}_{l}}{\partial x_{j}}\right)+\bar{v} \frac{\partial^{2} R_{i j}}{\partial x_{l}^{2}}-(2 \bar{v}+Q) \frac{\Delta R_{i j}}{s^{2}}
\end{gathered}
$$

This modeled equation displays the potential for describing various physical processes including creation, advection, turbulence diffusion, redistribution of Reynolds stress components, return to isotropy, creation due to $M_{i j}$, mean viscous diffusion, and decay. 


\section{$5.4 M_{i j}$ Transport Modeling}

The $M_{i j}$ tensor equation (4.10), within the restricted set of circumstances, reduces

to

$$
\begin{aligned}
& \frac{\partial M_{i j}}{\partial t}+M_{l j} \frac{\partial \bar{u}_{i}}{\partial x_{l}}+M_{i l} \frac{\partial \bar{u}_{l}}{\partial x_{j}}+\bar{u}_{l} \frac{\partial M_{i j}}{\partial x_{l}}+\overline{u_{l}^{\prime} \frac{\partial v^{\prime}}{\partial x_{j}} \frac{\partial u_{i}^{\prime}}{\partial x_{l}}}+\overline{u_{i}^{\prime} \frac{\partial}{\partial x_{j}}\left(u_{l}^{\prime} \frac{\partial v^{\prime}}{\partial x_{l}}\right)} \\
& =-\frac{\overline{\partial v^{\prime}}}{\partial x_{j}} \frac{\partial p^{\prime}}{\partial x_{i}}+Z_{j k}\left(\frac{\partial \bar{u}_{i}}{\partial x_{k}}+\frac{\partial \bar{u}_{k}}{\partial x_{i}}\right)+\bar{v} \frac{\partial^{2} M_{i j}}{\partial x_{l}^{2}}-2 \bar{v} \frac{\overline{\partial u_{i}^{\prime}}}{\partial x_{l}} \frac{\partial^{2} v^{\prime}}{\partial x_{l} \partial x_{j}} \\
& -\bar{v} u_{i}^{\prime} \frac{\partial^{3} v^{\prime}}{\partial x_{l}^{2} \partial x_{j}}+\overline{\frac{\partial v^{\prime}}{\partial x_{j}} \frac{\partial v^{\prime}}{\partial x_{k}}\left(\frac{\partial u_{i}^{\prime}}{\partial x_{k}}+\frac{\partial u_{k}^{\prime}}{\partial x_{i}}\right)}+\overline{v^{\prime} \frac{\partial v^{\prime}}{\partial x_{j}} \frac{\partial^{2} u_{i}^{\prime}}{\partial x_{k}^{2}}}
\end{aligned}
$$

The terms that need no modeling are:

Inertial term

$$
\frac{\partial M_{i j}}{\partial t}
$$

Mean flow coupling terms

$$
M_{l j} \frac{\partial \bar{u}_{i}}{\partial x_{l}} \& M_{i l} \frac{\partial \bar{u}_{l}}{\partial x_{j}}
$$

Advection term

$$
\bar{u}_{l} \frac{\partial M_{i j}}{\partial x_{l}}
$$

$Z_{i j}$ coupling to

the mean flow gradient

$$
Z_{j l}\left(\frac{\partial \bar{u}_{i}}{\partial x_{l}}+\frac{\partial \bar{u}_{l}}{\partial x_{i}}\right)
$$

Diffusion of $M_{i j}$

due to the mean viscosity

$$
\bar{v} \frac{\partial^{2} M_{i j}}{\partial x_{l}^{2}}
$$


The remaining terms, the triple correlations and the pressure-viscosity correlation, need to be modeled. The modeling of these terms follows the guidelines discussed for the Reynolds stress equation.

The triple correlation $\overline{u_{l}^{\prime} \frac{\partial v^{\prime}}{\partial x_{j}} \frac{\partial u_{i}^{\prime}}{\partial x_{l}}}$ can be rewritten

$$
\overline{u_{l}^{\prime} \frac{\partial v^{\prime}}{\partial x_{j}} \frac{\partial u_{i}^{\prime}}{\partial x_{l}}}=\frac{\partial}{\partial x_{l}}\left(\overline{u_{l}^{\prime} u_{i}^{\prime} \frac{\partial v^{\prime}}{\partial x_{j}}}\right)-\overline{u_{l}^{\prime} u_{i}^{\prime} \frac{\partial^{2} v^{\prime}}{\partial x_{j}^{2}}}
$$

The first term on the right side can be modeled using the gradient flux approximation

$$
\frac{\partial}{\partial x_{l}}\left(\overline{u_{l}^{\prime} u_{i}^{\prime} \frac{\partial v^{\prime}}{\partial x_{j}}}\right)=\frac{\partial}{\partial x_{l}}\left(\overline{u_{l}^{\prime} M_{i j}^{\prime}}\right) \cong \frac{\partial}{\partial x_{l}}\left[-C_{H} \frac{s}{\sqrt{K}} R_{l m} \frac{\partial M_{i j}}{\partial x_{m}}\right]
$$

in which $C_{H}$ is a dimensionless parameter. One possible model for the second term on the right side is the turbulence diffusion of the mean kinematic viscosity

$$
\overline{-u_{l}^{\prime} u_{i}^{\prime} \frac{\partial^{2} v^{\prime}}{\partial x_{j}^{2}}} \cong-C R_{l i} \frac{\partial^{2} \bar{v}}{\partial x_{j}^{2}}
$$

This term would vanish because the mean kinematic viscosity is constant. Another argument for neglecting this term is that the second order derivative of the fluctuating kinematic viscosity is negligible, so we have chosen to neglect this term.

The triple correlation $\overline{u_{i}^{\prime}} \frac{\partial}{\partial x_{j}}\left(u_{l}^{\prime} \frac{\partial v^{\prime}}{\partial x_{l}}\right)$ can be modeled using the gradient flux approximation

$$
\overline{u_{i}^{\prime} \frac{\partial}{\partial x_{j}}\left(u_{l}^{\prime} \frac{\partial v^{\prime}}{\partial x_{l}}\right)}=\overline{u_{i}^{\prime} \frac{\partial M_{l l}^{\prime}}{\partial x_{j}}} \cong-C \frac{s}{\sqrt{K}} R_{i m} \frac{\partial^{2} M_{l l}}{\partial x_{m} \partial x_{j}}
$$


This term vanishes because the contraction of the $M_{i j}$ tensor is zero, i.e. $M_{l l}=0$, for the currently restricted set of circumstances.

For the pressure-kinematic viscosity correlation $-\frac{\overline{\partial v^{\prime}}}{\partial x_{j}} \frac{\partial p^{\prime}}{\partial x_{i}}$ we proposed a return to isotropy model just as we did for the pressure-velocity correlation in the Reynolds stress tensor equation

$$
-\overline{\frac{\partial v^{\prime}}{\partial x_{j}} \frac{\partial p^{\prime}}{\partial x_{i}}} \cong C_{M} \frac{\sqrt{K}}{s}\left(\frac{2}{3} M_{l l} \delta_{i j}-M_{i j}\right)=-C_{M} \frac{\sqrt{K}}{s} M_{i j}
$$

where $C_{M}$ is a dimensionless parameter. Even though $M_{l l}$ vanishes, this model still behaves as a return to isotropy, but returning to the isotropic state of zero $M_{i j}$. Just as in the return to isotropy for the Reynolds stress tensor we propose that the return to isotropy is enhanced due to collisions of the nonhomogeneities, which are driven by the mean flow contortions in the system, so that

$$
-\overline{\frac{\partial v^{\prime}}{\partial x_{j}} \frac{\partial p^{\prime}}{\partial x_{i}}} \cong-\left(C_{M} \frac{\sqrt{K}}{s}+C_{H R} \sqrt{\frac{\partial \bar{u}_{k}}{\partial x_{l}} \frac{\partial \bar{u}_{k}}{\partial x_{l}}}\right) M_{i j}
$$

The correlation $-2 \bar{v} \overline{\frac{\partial u_{i}^{\prime}}{\partial x_{l}} \frac{\partial^{2} v^{\prime}}{\partial x_{l} \partial x_{j}}}$, looks similar to the decay term in the Reynolds stress tensor equation, so we propose the following decay model

$$
-2 \overline{\frac{\partial}{\partial u_{i}^{\prime}}} \frac{\partial^{2} v^{\prime}}{\partial x_{l}} \cong-2 \bar{v} \frac{\Delta}{s^{2}} M_{j} M_{i j}
$$


Even though there are no triple correlations to use to justify the addition of an enhancement to the decay term we added the enhancement to remain consistent with the decay term in the Reynolds stress equation

$$
-2 \bar{v} \overline{\frac{\partial u_{i}^{\prime}}{\partial x_{l}} \frac{\partial^{2} v^{\prime}}{\partial x_{l} \partial x_{j}}} \cong-(2 \bar{v}+\tilde{Q}) \frac{\Delta}{s^{2}} M_{i j}
$$

The double correlation $\bar{v} \overline{u_{i}^{\prime} \frac{\partial^{3} v^{\prime}}{\partial x_{l}^{2} \partial x_{j}}}$, is neglected because it is a third order derivative of the fluctuating kinematic viscosity.

The triple correlation $\overline{v^{\prime} \frac{\partial v^{\prime}}{\partial x_{j}} \frac{\partial^{2} u_{i}^{\prime}}{\partial x_{k}^{2}}}$ can be rewritten

$$
\overline{v^{\prime} \frac{\partial v^{\prime}}{\partial x_{j}} \frac{\partial^{2} u_{i}^{\prime}}{\partial x_{k}^{2}}}=\frac{1}{2}\left[\frac{\partial^{2}}{\partial x_{k}^{2}}\left(\overline{u_{i}^{\prime} \frac{\partial v^{\prime} v^{\prime}}{\partial x_{j}}}\right)-\overline{u_{i}^{\prime} \frac{\partial^{3} v^{\prime} v^{\prime}}{\partial x_{k}^{2} \partial x_{j}}}\right]
$$

These terms are in a form to use the gradient flux approximation

$$
\begin{aligned}
& \frac{1}{2}\left[\frac{\partial^{2}}{\partial x_{k}^{2}}\left(\overline{u_{i}^{\prime} \frac{\partial v^{\prime} v^{\prime}}{\partial x_{j}}}\right)-\overline{u_{i}^{\prime} \frac{\partial^{3} v^{\prime} v^{\prime}}{\partial x_{k}^{2} \partial x_{j}}}\right] \cong \frac{1}{2} \frac{\partial^{2}}{\partial x_{k}^{2}}\left[-C \frac{s}{\sqrt{K}} R_{i m} \frac{\partial}{\partial x_{m}}\left(\frac{\partial \overline{v^{\prime} v^{\prime}}}{\partial x_{j}}\right)\right] \\
& -\frac{1}{2}\left[-C \frac{s}{\sqrt{K}} R_{i m} \frac{\partial}{\partial x_{m}}\left(\frac{\partial^{3} \overline{v^{\prime} v^{\prime}}}{\partial x_{k}^{2} \partial x_{j}}\right)\right]
\end{aligned}
$$

Because $\overline{v^{\prime} v^{\prime}}$ is constant throughout time and space these terms vanish.

The triple correlation $\overline{\frac{\partial v^{\prime}}{\partial x_{j}} \frac{\partial v^{\prime}}{\partial x_{k}}\left(\frac{\partial u_{i}^{\prime}}{\partial x_{k}}+\frac{\partial u_{k}^{\prime}}{\partial x_{i}}\right)}$ is modeled by requiring the condition that the contraction of the $M_{i j}$ tensor is zero, i.e. $M_{l l}=0$. Contracting the $M_{i j}$ tensor equation, with the newly modeled terms and the triple correlation in question, results in the following 


$$
\frac{\partial M_{l l}}{\partial t}+\bar{u}_{k} \frac{\partial M_{l l}}{\partial x_{k}}+2 M_{n l} \frac{\partial \bar{u}_{l}}{\partial x_{n}}=Z_{n k}\left(\frac{\partial \bar{u}_{n}}{\partial x_{k}}+\frac{\partial \bar{u}_{k}}{\partial x_{n}}\right)+\overline{\frac{\partial v^{\prime}}{\partial x_{n}} \frac{\partial v^{\prime}}{\partial x_{k}}\left(\frac{\partial u_{n}^{\prime}}{\partial x_{k}}+\frac{\partial u_{k}^{\prime}}{\partial x_{n}}\right)}
$$

In order for $M_{l l}$ to remain equal to zero, we propose to write

$$
\overline{\frac{\partial v^{\prime}}{\partial x_{j}} \frac{\partial v^{\prime}}{\partial x_{k}}\left(\frac{\partial u_{i}^{\prime}}{\partial x_{k}}+\frac{\partial u_{k}^{\prime}}{\partial x_{i}}\right)}=\frac{\delta_{i j}}{3}\left[2 M_{n l} \frac{\partial \bar{u}_{l}}{\partial x_{n}}-Z_{n k}\left(\frac{\partial \bar{u}_{n}}{\partial x_{k}}+\frac{\partial \bar{u}_{k}}{\partial x_{n}}\right)\right]
$$

A possible addition to this model may be argued by using a gradient flux approximation

$$
\begin{gathered}
\overline{\frac{\partial v^{\prime}}{\partial x_{j}} \frac{\partial v^{\prime}}{\partial x_{k}}\left(\frac{\partial u_{i}^{\prime}}{\partial x_{k}}+\frac{\partial u_{k}^{\prime}}{\partial x_{i}}\right)}=\frac{\delta_{i j}}{3}\left[2 M_{n l} \frac{\partial \bar{u}_{l}}{\partial x_{n}}-Z_{n k}\left(\frac{\partial \bar{u}_{n}}{\partial x_{k}}+\frac{\partial \bar{u}_{k}}{\partial x_{n}}\right)\right] \\
-\frac{\partial}{\partial x_{k}}\left(C_{q} \frac{s}{\sqrt{K}} R_{i m}\right) \frac{\partial Z_{j k}}{\partial x_{m}}-\frac{\partial}{\partial x_{i}}\left(C_{q} \frac{s}{\sqrt{K}} R_{k m}\right) \frac{\partial Z_{j k}}{\partial x_{m}}
\end{gathered}
$$

The two additional terms represent the coupling of $Z_{i j}$ to the turbulent kinetic energy. These two terms may be negligible because they are higher order terms, so we have not included them in our model for the $M_{i j}$ equation.

Substituting the modeled terms for the exact terms results in a modeled, but closed $M_{i j}$ tensor equation

$$
\begin{aligned}
\frac{\partial M_{i j}}{\partial t}+M_{l j} \frac{\partial \bar{u}_{i}}{\partial x_{l}} & +M_{i l} \frac{\partial \bar{u}_{l}}{\partial x_{j}}-\frac{2 \delta_{i j}}{3} M_{n l} \frac{\partial \bar{u}_{l}}{\partial x_{n}}+\bar{u}_{l} \frac{\partial M_{i j}}{\partial x_{l}}-\frac{\partial}{\partial x_{l}}\left[C_{H} \frac{s}{\sqrt{K}} R_{l m} \frac{\partial M_{i j}}{\partial x_{m}}\right] \\
= & -\left[(2 \bar{v}+\tilde{Q}) \frac{\Delta}{s^{2}}+C_{M} \frac{\sqrt{K}}{s}+C_{H R} \sqrt{\frac{\partial \bar{u}_{k}}{\partial x_{l}} \frac{\partial \bar{u}_{k}}{\partial x_{l}}}\right] M_{i j} \\
+ & Z_{j k}\left(\frac{\partial \bar{u}_{i}}{\partial x_{k}}+\frac{\partial \bar{u}_{k}}{\partial x_{i}}\right)-\frac{\delta_{i j}}{3} Z_{n k}\left(\frac{\partial \bar{u}_{n}}{\partial x_{k}}+\frac{\partial \bar{u}_{k}}{\partial x_{n}}\right)+\bar{v} \frac{\partial^{2} M_{i j}}{\partial x_{l}^{2}}
\end{aligned}
$$


This modeled equation displays the potential for describing various physical processes including creation, advection, turbulence diffusion, decay, redistribution of $M_{i j}$ components, return to isotropy, creation due to the $Z_{i j}$ tensor, and mean viscous diffusion.

\section{$5.5 Z_{i j}$ Transport Modeling}

The $Z_{i j}$ tensor equation (4.21), within the restricted set of circumstances, reduces

to

$$
\frac{\partial Z_{i j}}{\partial t}+Z_{i l} \frac{\partial \bar{u}_{l}}{\partial x_{j}}+Z_{j l} \frac{\partial \bar{u}_{l}}{\partial x_{i}}+\bar{u}_{l} \frac{\partial Z_{i j}}{\partial x_{l}}+\overline{\frac{\partial v^{\prime}}{\partial x_{i}} \frac{\partial u_{l}^{\prime}}{\partial x_{j}} \frac{\partial v^{\prime}}{\partial x_{l}}}+\overline{\frac{\partial v^{\prime}}{\partial x_{j}} \frac{\partial u_{l}^{\prime}}{\partial x_{i}} \frac{\partial v^{\prime}}{\partial x_{l}}}+\overline{u_{l}^{\prime} \frac{\partial}{\partial x_{l}}\left(\frac{\partial v^{\prime}}{\partial x_{i}} \frac{\partial v^{\prime}}{\partial x_{j}}\right)}=0
$$

The terms that need no modeling are:

Inertial term

$$
\frac{\partial Z_{i j}}{\partial t}
$$

Mean flow coupling terms

$$
Z_{i l} \frac{\partial \bar{u}_{l}}{\partial x_{j}} \& Z_{j l} \frac{\partial \bar{u}_{l}}{\partial x_{i}}
$$

Advection term

$$
\bar{u}_{l} \frac{\partial Z_{i j}}{\partial x_{l}}
$$

The remaining triple correlations need to be modeled. The modeling of these terms follows the guidelines for terms in the Reynolds stress equation and in the $M_{i j}$ tensor equation.

The triple correlation $\overline{u_{l}^{\prime}} \frac{\partial}{\partial x_{l}}\left(\frac{\partial v^{\prime}}{\partial x_{i}} \frac{\partial v^{\prime}}{\partial x_{j}}\right)$ is in a form to use the gradient flux approximation 


$$
\overline{u_{l}^{\prime} \frac{\partial}{\partial x_{l}}\left(\frac{\partial v^{\prime}}{\partial x_{i}} \frac{\partial v^{\prime}}{\partial x_{j}}\right)} \cong-C_{X} \frac{s}{\sqrt{K}} R_{l m} \frac{\partial^{2} Z_{i j}}{\partial x_{m} \partial x_{l}}
$$

where $C_{X}$ is a dimensionless parameter. This term is a turbulence diffusion of $Z_{i j}$, but to be consistent with the turbulence diffusion of $R_{i j}$ and $M_{i j}$ we model this term as

$$
\overline{u_{l}^{\prime} \frac{\partial}{\partial x_{l}}\left(\frac{\partial v^{\prime}}{\partial x_{i}} \frac{\partial v^{\prime}}{\partial x_{j}}\right)} \cong-\frac{\partial}{\partial x_{l}}\left(C_{X} \frac{s}{\sqrt{K}} R_{l m} \frac{\partial Z_{i j}}{\partial x_{m}}\right)
$$

$Z_{i j}$ describes the configuration of the nonhomogeneities and from physical intuition we believe the nonhomogeneities have a limit to how much they can be contorted. We believe the nonhomogeneities have a tendency to return to an isotropic state while being stretched out, so we have proposed a return to isotropy for the two triple correlations $\overline{\frac{\partial v^{\prime}}{\partial x_{i}} \frac{\partial u_{l}^{\prime}}{\partial x_{j}} \frac{\partial v^{\prime}}{\partial x_{l}}}+\frac{\overline{\partial v^{\prime}}}{\partial x_{j}} \frac{\partial u_{l}^{\prime}}{\partial x_{i}} \frac{\partial v^{\prime}}{\partial x_{l}}$. In addition to a return to isotropy model we propose that the contraction of the $Z_{i j}$ tensor is constant, which requires that the transport equation of $Z_{n n}$ is zero. This implies that if a nonhomogeniety is stretched in one direction it contracts in the other two directions, so that the fluid elements are only rearranged configurationally within the constraint that the kinematic viscosity of a given fluid element does not change during crenulation, as seen in Figure 5.1 

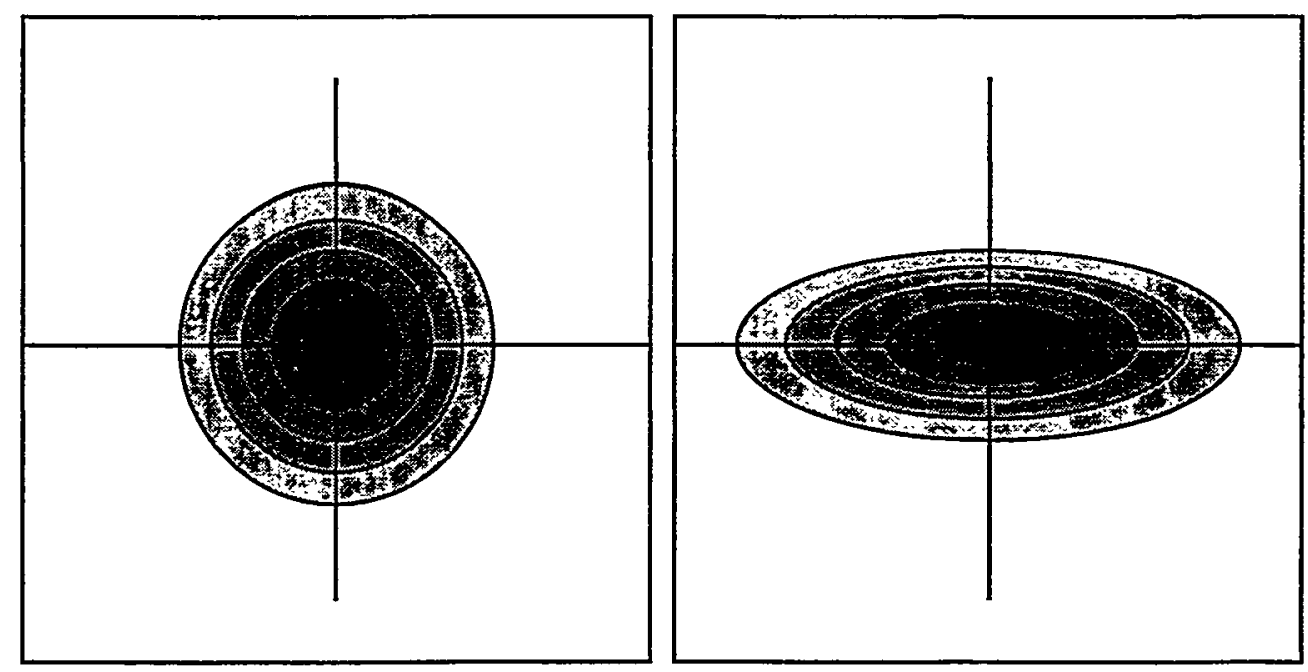

Figure 5.1: Stretching of a Nonhomogeneity

The proposed model for the two triple correlations has a return to isotropy term and a tensor $D_{i j}$ to be determined from the postulate $Z_{n n}=$ constant.

$$
\overline{\frac{\partial v^{\prime}}{\partial x_{i}} \frac{\partial u_{l}^{\prime}}{\partial x_{j}} \frac{\partial v^{\prime}}{\partial x_{l}}}+\overline{\frac{\partial v^{\prime}}{\partial x_{j}} \frac{\partial u_{l}^{\prime}}{\partial x_{i}} \frac{\partial v^{\prime}}{\partial x_{l}}} \cong\left(C_{Z} \frac{\sqrt{K}}{s}+C_{H R} \sqrt{\frac{\partial \bar{u}_{k}}{\partial x_{l}} \frac{\partial \bar{u}_{k}}{\partial x_{l}}}\right)\left(Z_{i j}-\frac{\delta_{i j}}{3} Z_{n n}\right)+D_{i j}
$$

where $C_{Z}$ is a dimensionless parameter. The return to isotropy model has the same enhancement as in the Reynolds stress equation and in the $M_{i j}$ equation. Contracting the $Z_{i j}$ equation, with the newly modeled terms, results in the following

$$
\frac{\partial Z_{n n}}{\partial t}+2 Z_{n l} \frac{\partial \bar{u}_{l}}{\partial x_{n}}+\bar{u}_{l} \frac{\partial Z_{n n}}{\partial x_{l}}=D_{n n}+\frac{\partial}{\partial x_{l}}\left(C_{X} \frac{s}{\sqrt{K}} R_{l m} \frac{\partial Z_{n n}}{\partial x_{m}}\right)
$$

which shows that we require

$$
D_{i j}=\frac{\delta_{i j}}{3}\left[2 Z_{n l} \frac{\partial \bar{u}_{l}}{\partial x_{n}}-\frac{\partial}{\partial x_{l}}\left(C_{X} \frac{s}{\sqrt{K}} R_{l m} \frac{\partial Z_{n n}}{\partial x_{m}}\right)\right]
$$


Substituting the modeled terms for the exact terms results in a modeled, but closed $Z_{i j}$ tensor equation

$$
\begin{gathered}
\frac{\partial Z_{i j}}{\partial t}+Z_{i l} \frac{\partial \bar{u}_{l}}{\partial x_{j}}+Z_{j l} \frac{\partial \bar{u}_{l}}{\partial x_{i}}-\frac{2 \delta_{i j}}{3} Z_{n l} \frac{\partial \bar{u}_{l}}{\partial x_{n}}+\bar{u}_{l} \frac{\partial Z_{i j}}{\partial x_{l}} \\
=\left(C_{Z} \frac{\sqrt{K}}{s}+C_{H R} \sqrt{\frac{\partial \bar{u}_{k}}{\partial x_{l}} \frac{\partial \bar{u}_{k}}{\partial x_{l}}}\right)\left(Z_{i j}-\frac{\delta_{i j}}{3} Z_{n n}\right) \\
+\frac{\partial}{\partial x_{l}}\left(C_{X} \frac{s}{\sqrt{K}} R_{l m} \frac{\partial Z_{i j}}{\partial x_{m}}\right)-\frac{\delta_{i j}}{3} \frac{\partial}{\partial x_{l}}\left(C_{X} \frac{s}{\sqrt{K}} R_{l m} \frac{\partial Z_{n n}}{\partial x_{m}}\right)
\end{gathered}
$$

This modeled equation displays the potential for describing various physical processes including creation, advection, return to isotropy, and turbulence diffusion.

\subsection{Distribution of Energy (Modeled)}

The modeling of the mean internal energy equation and of the turbulent kinetic energy equation are direct consequence of the modeling in the transport equation of the Reynolds stress tensor. The mean internal energy equation (3.7), within the restricted set of circumstances, reduces to

$$
\begin{gathered}
\frac{\partial \bar{e}}{\partial t}+\bar{u}_{l} \frac{\partial \bar{e}}{\partial x_{l}}=\bar{v}\left(\frac{\partial \bar{u}_{i}}{\partial x_{l}}+\frac{\partial \bar{u}_{l}}{\partial x_{i}}\right) \frac{\partial \bar{u}_{i}}{\partial x_{l}}+\bar{v}\left(\overline{\frac{\partial u_{i}^{\prime}}{\partial x_{l}} \frac{\partial u_{i}^{\prime}}{\partial x_{l}}}+\frac{\partial^{2} R_{l i}}{\partial x_{l} \partial x_{i}}\right) \\
-2 M_{i l}\left(\frac{\partial \bar{u}_{i}}{\partial x_{l}}+\frac{\partial \bar{u}_{l}}{\partial x_{i}}\right)+\frac{\partial u_{i}^{\prime} \frac{\partial u_{i}^{\prime}}{\partial x_{l}}}{\partial x_{l}}+\frac{\partial u_{l}^{\prime} \frac{\partial u_{i}^{\prime}}{\partial x_{i}} \frac{v^{\prime}}{\partial x_{l}}}{}
\end{gathered}
$$

The terms that need no modeling are:

Inertial term

$$
\frac{\partial \bar{e}}{\partial t}
$$

Advection term

$$
\bar{u}_{l} \frac{\partial \bar{e}}{\partial x_{l}}
$$


Dissipation of the mean

flow due to the mean

kinematic viscosity $\quad \bar{v}\left(\frac{\partial \bar{u}_{i}}{\partial x_{l}}+\frac{\partial \bar{u}_{l}}{\partial x_{i}}\right) \frac{\partial \bar{u}_{i}}{\partial x_{l}}$

Diffusion of the Reynolds stress

due to the mean viscosity

$$
\bar{v} \frac{\partial^{2} R_{l i}}{\partial x_{l} \partial x_{i}}
$$

$M_{i j}$ coupling to

the mean flow gradient $\quad-2 M_{i l}\left(\frac{\partial \bar{u}_{i}}{\partial x_{l}}+\frac{\partial \bar{u}_{l}}{\partial x_{i}}\right)$

The remaining terms, the double velocity correlations and the triple correlations involving a kinematic viscosity fluctuation are terms that need to be modeled. The modeling of these terms must follow exactly the same procedures as in the modeling of the Reynolds stress equation. This is the case because sinks to the turbulence energy are sources to the mean internal energy.

The double velocity correlation $\bar{v} \overline{\frac{\partial u_{i}^{\prime}}{\partial x_{l}} \frac{\partial u_{i}^{\prime}}{\partial x_{l}}}$ in the mean internal energy equation has a counterpart in the Reynolds stress tensor equation $-2 \bar{v} \frac{\overline{\partial u_{i}^{\prime}}}{\partial x_{l}} \frac{\partial u_{j}^{\prime}}{\partial x_{l}}$. The model for the term in the Reynolds stress equation is

$$
-2 \bar{v} \frac{\partial u_{i}^{\prime}}{\partial x_{l}} \frac{\partial u_{j}^{\prime}}{\partial x_{l}} \cong-(2 \bar{v}+Q) \frac{\Delta R_{i j}}{s^{2}}
$$

Contracting this model gives us the model for the double correlation in the mean internal energy equation 


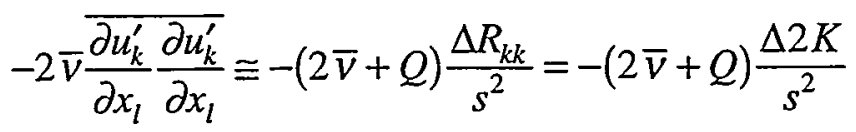

or

$$
\overline{\bar{v}} \overline{\frac{\partial u_{k}^{\prime}}{\partial x_{l}} \frac{\partial u_{k}^{\prime}}{\partial x_{l}}} \cong(2 \bar{v}+Q) \frac{\Delta R_{k k}}{2 s^{2}}=(2 \bar{v}+Q) \frac{\Delta K}{s^{2}}
$$

The triple correlations $\overline{v^{\prime} \frac{\partial u_{i}^{\prime}}{\partial x_{l}} \frac{\partial u_{i}^{\prime}}{\partial x_{l}}}$ and $\overline{v^{\prime} \frac{\partial u_{l}^{\prime}}{\partial x_{i}} \frac{\partial u_{i}^{\prime}}{\partial x_{l}}}$ in the mean internal energy equation have their counterparts (in an indirect way) in the Reynolds stress transport terms $\overline{u_{j}^{\prime} \frac{\partial}{\partial x_{l}}\left[v^{\prime}\left(\frac{\partial u_{i}^{\prime}}{\partial x_{l}}+\frac{\partial u_{l}^{\prime}}{\partial x_{i}}\right)\right]}$ and $u_{i}^{\prime} \frac{\partial}{\partial x_{l}}\left[v^{\prime}\left(\frac{\partial u_{j}^{\prime}}{\partial x_{l}}+\frac{\partial u_{l}^{\prime}}{\partial x_{j}}\right)\right]$. In the Reynolds stress equation we simply neglected these triple correlation terms, so we have done the same with the triple correlation terms in the mean internal energy equation.

Substituting the modeled term for the exact term results in a modeled, but closed mean internal energy equation

$$
\begin{gathered}
\frac{\partial \bar{e}}{\partial t}+\bar{u}_{l} \frac{\partial \bar{e}}{\partial x_{l}}=\bar{v}\left(\frac{\partial \bar{u}_{i}}{\partial x_{l}}+\frac{\partial \bar{u}_{l}}{\partial x_{i}}\right) \frac{\partial \bar{u}_{i}}{\partial x_{l}}+(2 \bar{v}+Q) \frac{\Delta K}{s^{2}} \\
+\bar{v} \frac{\partial^{2} R_{l i}}{\partial x_{l} \partial x_{i}}-2 M_{i l}\left(\frac{\partial \bar{u}_{i}}{\partial x_{l}}+\frac{\partial \bar{u}_{l}}{\partial x_{i}}\right)
\end{gathered}
$$

This modeled equation displays the potential for describing various physical processes including advection, dissipation of the mean flow energy, dissipation of the turbulence energy, mean viscous diffusion, and alterations due to the $M_{i j}$ tensor.

The mean kinetic energy equation (4.24) does not have to be modeled

$$
\begin{gathered}
\frac{\partial K E}{\partial t}+\bar{u}_{l} \frac{\partial K E}{\partial x_{l}}+\bar{u}_{i} \frac{\partial R_{i l}}{\partial x_{l}}=-\bar{u}_{i} \frac{\partial \bar{p}}{\partial x_{i}} \\
+\bar{v} \frac{\partial^{2} K E}{\partial x_{l}^{2}}-\bar{v} \frac{\partial \bar{u}_{i}}{\partial x_{l}} \frac{\partial \bar{u}_{i}}{\partial x_{l}}-\bar{u}_{i} \frac{\partial\left(M_{i l}+M_{l i}\right)}{\partial x_{l}}
\end{gathered}
$$


The turbulent kinetic energy equation does not have to be modeled because it is the contraction of the Reynolds stress tensor equation, and the Reynolds stress tensor equation has already been modeled. Contracting the Reynolds stress equation results in the following turbulence energy equation

$$
\begin{gathered}
\frac{\partial K}{\partial t}+R_{n l} \frac{\partial \bar{u}_{n}}{\partial x_{l}}+\bar{u}_{l} \frac{\partial K}{\partial x_{l}}=\frac{\partial}{\partial x_{l}}\left[C_{D} \frac{s}{\sqrt{K}} R_{l m} \frac{\partial K}{\partial x_{m}}\right] \\
+M_{n l}\left(\frac{\partial \bar{u}_{n}}{\partial x_{l}}+\frac{\partial \bar{u}_{l}}{\partial x_{n}}\right)+\bar{v} \frac{\partial^{2} K}{\partial x_{l}^{2}}-(2 \bar{v}+Q) \frac{\Delta K}{s^{2}}
\end{gathered}
$$

\subsection{Summary of Modeled Transport Equations}

Incompressibility condition equation (3.5)

$$
\frac{\partial \bar{u}_{l}}{\partial x_{l}}=0
$$

Mean momentum equation (3.6)

$$
\frac{\partial \bar{u}_{i}}{\partial t}+\frac{\partial \bar{u}_{i} \bar{u}_{l}}{\partial x_{l}}+\frac{\partial R_{i l}}{\partial x_{l}}=-\frac{\partial \bar{p}}{\partial x_{i}}+\frac{\partial}{\partial x_{l}}\left[\bar{v}\left(\frac{\partial \bar{u}_{i}}{\partial x_{l}}+\frac{\partial \bar{u}_{l}}{\partial x_{i}}\right)\right]-\frac{\partial\left(M_{i l}+M_{l i}\right)}{\partial x_{l}}
$$

Reynolds stress transport equation (5.6)

$$
\begin{gathered}
\frac{\partial R_{i j}}{\partial t}+R_{j l} \frac{\partial \bar{u}_{i}}{\partial x_{l}}+R_{i l} \frac{\partial \bar{u}_{j}}{\partial x_{l}}+\bar{u}_{l} \frac{\partial R_{i j}}{\partial x_{l}}=\frac{\partial}{\partial x_{l}}\left[C_{D} \frac{s}{\sqrt{K}} R_{l m} \frac{\partial R_{i j}}{\partial x_{m}}\right] \\
+C_{B}\left(R_{i l} \frac{\partial \bar{u}_{j}}{\partial x_{l}}+R_{j l} \frac{\partial \bar{u}_{i}}{\partial x_{l}}-\frac{2}{3} R_{k l} \frac{\partial \bar{u}_{k}}{\partial x_{l}} \delta_{i j}\right)+\left(C_{R} \frac{\sqrt{K}}{s}+C_{H R} \sqrt{\frac{\partial \bar{u}_{k}}{\partial x_{l}} \frac{\partial \bar{u}_{k}}{\partial x_{l}}}\right)\left(\frac{2}{3} K \delta_{i j}-R_{i j}\right) \\
+M_{j l}\left(\frac{\partial \bar{u}_{i}}{\partial x_{l}}+\frac{\partial \bar{u}_{l}}{\partial x_{i}}\right)+M_{i l}\left(\frac{\partial \bar{u}_{j}}{\partial x_{l}}+\frac{\partial \bar{u}_{l}}{\partial x_{j}}\right)+\bar{v} \frac{\partial^{2} R_{i j}}{\partial x_{l}^{2}}-(2 \bar{v}+\tilde{Q}) \frac{\Delta R_{i j}}{s^{2}}
\end{gathered}
$$


$M_{i j}$ transport equation (5.11)

$$
\begin{aligned}
\frac{\partial M_{i j}}{\partial t}+M_{l j} \frac{\partial \bar{u}_{i}}{\partial x_{l}}+ & M_{i l} \frac{\partial \bar{u}_{l}}{\partial x_{j}}-\frac{2 \delta_{i j}}{3} M_{n l} \frac{\partial \bar{u}_{l}}{\partial x_{n}}+\bar{u}_{l} \frac{\partial M_{i j}}{\partial x_{l}}-\frac{\partial}{\partial x_{l}}\left[C_{H} \frac{s}{\sqrt{K}} R_{l m} \frac{\partial M_{i j}}{\partial x_{m}}\right] \\
= & -\left[(2 \bar{v}+Q) \frac{\Delta}{s^{2}}+C_{M} \frac{\sqrt{K}}{s}+C_{H R} \sqrt{\frac{\partial \bar{u}_{k}}{\partial x_{l}} \frac{\partial \bar{u}_{k}}{\partial x_{l}}}\right] M_{i j} \\
+ & Z_{j k}\left(\frac{\partial \bar{u}_{i}}{\partial x_{k}}+\frac{\partial \bar{u}_{k}}{\partial x_{i}}\right)-\frac{\delta_{i j}}{3} Z_{n k}\left(\frac{\partial \bar{u}_{n}}{\partial x_{k}}+\frac{\partial \bar{u}_{k}}{\partial x_{n}}\right)+\bar{v} \frac{\partial^{2} M_{i j}}{\partial x_{l}^{2}}
\end{aligned}
$$

$Z_{i j}$ transport equation (5.14)

$$
\begin{gathered}
\frac{\partial Z_{i j}}{\partial t}+Z_{i l} \frac{\partial \bar{u}_{l}}{\partial x_{j}}+Z_{j l} \frac{\partial \bar{u}_{l}}{\partial x_{i}}-\frac{2 \delta_{i j}}{3} Z_{n l} \frac{\partial \bar{u}_{l}}{\partial x_{n}}+\bar{u}_{l} \frac{\partial Z_{i j}}{\partial x_{l}} \\
=\left(C_{Z} \frac{\sqrt{K}}{s}+C_{H R} \sqrt{\frac{\partial \bar{u}_{k}}{\partial x_{l}} \frac{\partial \bar{u}_{k}}{\partial x_{l}}}\right)\left(\frac{\delta_{i j}}{3} Z_{n n}-Z_{i j}\right) \\
+\frac{\partial}{\partial x_{l}}\left(C_{X} \frac{s}{\sqrt{K}} R_{l m} \frac{\partial Z_{i j}}{\partial x_{m}}\right)-\frac{\delta_{i j}}{3} \frac{\partial}{\partial x_{l}}\left(C_{X} \frac{s}{\sqrt{K}} R_{l m} \frac{\partial Z_{n n}}{\partial x_{m}}\right)
\end{gathered}
$$

Mean internal energy equation (5.16)

$$
\begin{aligned}
\frac{\partial \bar{e}}{\partial t}+\bar{u}_{l} \frac{\partial \bar{e}}{\partial x_{l}} & =\bar{v}\left(\frac{\partial \bar{u}_{i}}{\partial x_{l}}+\frac{\partial \bar{u}_{l}}{\partial x_{i}}\right) \frac{\partial \bar{u}_{i}}{\partial x_{l}}+(2 \bar{v}+Q) \frac{\Delta K}{s^{2}} \\
+ & \bar{v} \frac{\partial^{2} R_{l i}}{\partial x_{l} \partial x_{i}}-2 M_{i l}\left(\frac{\partial \bar{u}_{i}}{\partial x_{l}}+\frac{\partial \bar{u}_{l}}{\partial x_{i}}\right)
\end{aligned}
$$

Mean kinetic energy equation (5.17)

$$
\begin{gathered}
\frac{\partial K E}{\partial t}+\bar{u}_{l} \frac{\partial K E}{\partial x_{l}}+\bar{u}_{i} \frac{\partial R_{i l}}{\partial x_{l}}=-\bar{u}_{i} \frac{\partial \bar{p}}{\partial x_{i}} \\
+\bar{v} \frac{\partial^{2} K E}{\partial x_{l}^{2}}-\bar{v} \frac{\partial \bar{u}_{i}}{\partial x_{l}} \frac{\partial \bar{u}_{i}}{\partial x_{l}}-\bar{u}_{i} \frac{\partial\left(M_{i l}+M_{l i}\right)}{\partial x_{l}}
\end{gathered}
$$


Turbulent kinetic energy equation (5.18)

$$
\begin{gathered}
\frac{\partial K}{\partial t}+R_{n l} \frac{\partial \bar{u}_{n}}{\partial x_{l}}+\bar{u}_{l} \frac{\partial K}{\partial x_{l}}=\frac{\partial}{\partial x_{l}}\left[C_{D} \frac{s}{\sqrt{K}} R_{l m} \frac{\partial K}{\partial x_{m}}\right] \\
+M_{n l}\left(\frac{\partial \bar{u}_{n}}{\partial x_{l}}+\frac{\partial \bar{u}_{l}}{\partial x_{n}}\right)+\bar{v} \frac{\partial^{2} K}{\partial x_{l}^{2}}-(2 \bar{v}+Q) \frac{\Delta K}{s^{2}}
\end{gathered}
$$

These equations are the modeled but closed set of transport equations that govern the currently restricted set of circumstances for crenulation in this thesis. In more general circumstances, $\bar{v}$ will not necessarily be constant, and a more extensive set of equations will be required. At this stage in the development, we test the more restrictive set of governing equations in order to see if this approach has merit. 


\section{Chapter 6}

\section{Modeled Transport Equations in a Spherical Coordinate System}

Crenulation most typically occurs in a converging circumstance, and to illustrate the crenulative process we have chosen the stochastically steady convergence of fluid through a stationary spherical shell. All of the mean quantities in the crenulative material are independent of angle, and there is no mean angular motion of the fluid. This means the governing equations are independent of $\theta$ and $\phi$ in a spherical coordinate system, Fig.

6.1. It must be emphasized that the fluctuations from the mean are fully three dimensional, whereas the moments of correlated fluctuations vary only with radius and time. The modeled governing equations are transformed from Cartesian tensor form to vector form, then to a spherical form for individual components. The vector notation is only a convenient intermediate step in obtaining the individual components of the governing equations in spherical coordinates.

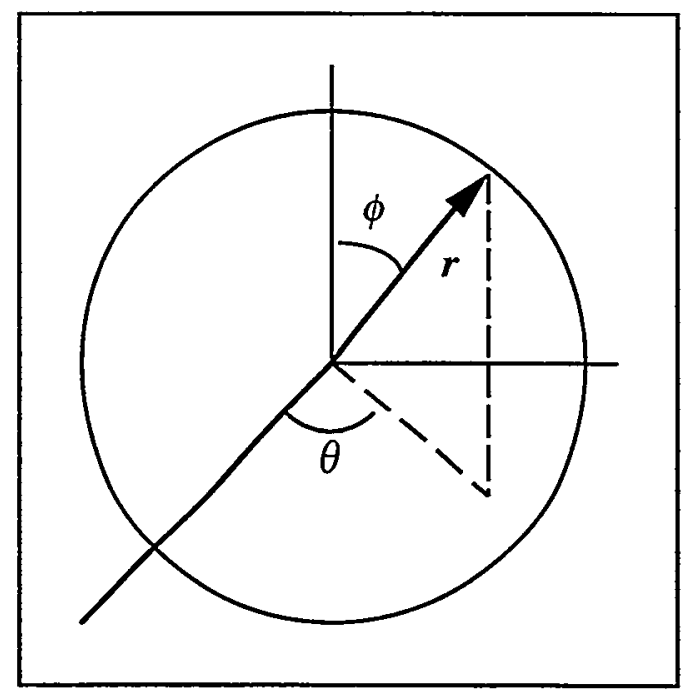

Figure 6.1: Spherical Coordinate System 
In addition to the vector notation an approximation for the Reynolds stress in the turbulence diffusion coefficient is substituted in all of the transport equations

$$
2 K \delta_{l m} \cong R_{l m}
$$

This approximation alleviates some of the complexities associated with a spherical coordinate system.

\subsection{Governing Equations in Vector Notation}

For the vector notation equations we write both vectors and tensors in bold type, so that $\bar{u}, R, M$, and $Z$ are the mean velocity vector field, the Reynolds stress tensor $R_{i j}$, the $M_{i j}$ tensor, and the $Z_{i j}$ tensor, respectively, while $\delta$ denotes the Kronecker Delta tensor, $\delta_{i j}$.

The modeled governing equations in vector notation are as follows:

The incompressibility condition, equation (3.5)

$$
\nabla \cdot \bar{u}=0
$$

The mean momentum equation (3.6)

$$
\frac{\partial \bar{u}}{\partial t}+\overline{\boldsymbol{u}} \cdot \nabla \overline{\boldsymbol{u}}+\nabla \cdot \boldsymbol{R}=-\nabla \bar{p}+\nabla \cdot\left\{\bar{v}\left[\nabla \overline{\boldsymbol{u}}+(\nabla \overline{\boldsymbol{u}})^{T}\right]\right\}-\left[\nabla \cdot \boldsymbol{M}+(\nabla \cdot \boldsymbol{M})^{T}\right]
$$


Reynolds stress transport equation (5.6)

$$
\begin{gathered}
\frac{\partial \boldsymbol{R}}{\partial t}+\boldsymbol{R} \cdot \nabla \overline{\boldsymbol{u}}+(\boldsymbol{R} \cdot \nabla \overline{\boldsymbol{u}})^{T}+\overline{\boldsymbol{u}} \cdot \nabla \boldsymbol{R}=\nabla \cdot\left(2 C_{D} s \sqrt{K} \nabla \boldsymbol{R}\right) \\
+C_{B}\left[\boldsymbol{R} \cdot \nabla \overline{\boldsymbol{u}}+(\boldsymbol{R} \cdot \nabla \overline{\boldsymbol{u}})^{T}-\frac{2 \delta}{3}(\boldsymbol{R}: \nabla \overline{\boldsymbol{u}})\right]+\left(C_{R} \frac{\sqrt{K}}{s}+C_{H R} \sqrt{\nabla \overline{\boldsymbol{u}}: \nabla \overline{\boldsymbol{u}}}\right)\left(\frac{2 \delta}{3} K-\boldsymbol{R}\right) \\
+\boldsymbol{M} \cdot \nabla \overline{\boldsymbol{u}}+\boldsymbol{M} \cdot(\nabla \overline{\boldsymbol{u}})^{T}+(\boldsymbol{M} \cdot \nabla \overline{\boldsymbol{u}})^{T}+\left[\boldsymbol{M} \cdot(\nabla \overline{\boldsymbol{u}})^{T}\right]^{T}+\bar{v} \nabla \cdot \nabla \boldsymbol{R}-(2 \bar{v}+Q) \frac{\Delta \boldsymbol{R}}{s^{2}}
\end{gathered}
$$

$M_{i j}$ transport equation (5.11)

$$
\begin{aligned}
& \frac{\partial M}{\partial t}+M^{T} \cdot \nabla \overline{\boldsymbol{u}}+\left[\boldsymbol{M} \cdot(\nabla \overline{\boldsymbol{u}})^{T}\right]^{T}-\frac{2 \delta}{3}\left[\boldsymbol{M}:(\nabla \overline{\boldsymbol{u}})^{T}\right]+\overline{\boldsymbol{u}} \cdot \nabla M-\nabla \cdot\left(2 C_{H} s \sqrt{K} \nabla M\right) \\
&=-\left[(2 \bar{v}+Q) \frac{\Delta}{s^{2}}+C_{M} \frac{\sqrt{K}}{s}+C_{H R} \sqrt{\nabla \bar{u}: \nabla \bar{u}}\right] M \\
&+Z \cdot \nabla \overline{\boldsymbol{u}}+Z \cdot(\nabla \bar{u})^{T}-\frac{\delta}{3}\left[Z: \nabla \overline{\boldsymbol{u}}+Z:(\nabla \bar{u})^{T}\right]+\bar{v} \nabla \cdot \nabla M
\end{aligned}
$$

$Z_{i j}$ transport equation (5.14)

$$
\begin{aligned}
\frac{\partial Z}{\partial t}+ & {\left[Z \cdot(\nabla \overline{\boldsymbol{u}})^{T}\right]^{T}+Z \cdot(\nabla \bar{u})^{T}-\frac{2 \delta}{3}\left[Z:(\nabla \bar{u})^{T}\right]+\bar{u} \cdot \nabla Z } \\
= & \left(C_{Z} \frac{\sqrt{K}}{s}+C_{H R} \sqrt{\nabla \overline{\boldsymbol{u}}: \nabla \bar{u}}\right)\left(\frac{\delta}{3} Z_{\text {trace }}-Z\right) \\
+ & \nabla \cdot\left(2 C_{X} s \sqrt{K} \nabla Z\right)-\frac{\delta}{3} \nabla \cdot\left(2 C_{X} s \sqrt{K} \nabla Z_{\text {trace }}\right)
\end{aligned}
$$

where $Z_{\text {trace }}$ is the trace of the tensor $\boldsymbol{Z}$.

Mean internal energy equation (5.16)

$$
\begin{gathered}
\frac{\partial \bar{e}}{\partial t}+\overline{\boldsymbol{u}} \cdot \nabla \bar{e}=\bar{v}\left[\nabla \overline{\boldsymbol{u}}: \nabla \overline{\boldsymbol{u}}+(\nabla \overline{\boldsymbol{u}})^{T}: \nabla \overline{\boldsymbol{u}}\right]+(2 \bar{v}+Q) \frac{\Delta K}{s^{2}} \\
+\bar{v} \nabla \cdot(\nabla \cdot \boldsymbol{R})-2\left[\boldsymbol{M}: \nabla \overline{\boldsymbol{u}}+\boldsymbol{M}:(\nabla \bar{u})^{T}\right]
\end{gathered}
$$


Mean kinetic energy equation (5.17)

$$
\begin{gathered}
\frac{\partial K E}{\partial t}+\bar{u} \cdot \nabla K E+\bar{u} \cdot(\nabla \cdot R)=-\bar{u} \cdot \nabla \bar{p} \\
+\bar{v} \nabla \cdot \nabla K E-\bar{v} \nabla \bar{u}: \nabla \bar{u}-\bar{u} \cdot(\nabla \cdot M)-\bar{u} \cdot\left(\nabla \cdot M^{T}\right)
\end{gathered}
$$

Turbulent kinetic energy equation (5.18)

$$
\begin{gathered}
\frac{\partial K}{\partial t}+\boldsymbol{R}: \nabla \overline{\boldsymbol{u}}+\overline{\boldsymbol{u}} \cdot \nabla K=\nabla \cdot\left(2 C_{D} s \sqrt{K} \nabla K\right) \\
+\boldsymbol{M}: \nabla \overline{\boldsymbol{u}}+\boldsymbol{M}:(\nabla \overline{\boldsymbol{u}})^{T}+\bar{v} \nabla \cdot \nabla K-(2 \bar{v}+Q) \frac{\Delta K}{s^{2}}
\end{gathered}
$$

\subsection{Individual Components of the Incompressibility Condition and the Mean Momentum Equation}

The incompressible condition for the motion of fluid in a spherical shell is

$$
\frac{1}{r^{2}} \frac{\partial}{\partial r}\left(r^{2} \bar{u}_{r}\right)=0
$$

With this simplified incompressibility condition we can solve for the mean radial velocity directly

$$
\bar{u}_{r}=\frac{B(t)}{r^{2}}
$$

where $B(t)$ is a function of time, only, and has the dimensions of length ${ }^{3} /$ time. For the $^{2}$ stochastically steady motion through a stationary shell, $B(t)=B$ is constant. 
The mean momentum equation simplifies to the mean equation for the radial velocity component only because there is no mean angular motion or angular dependence of the variables

$$
\begin{gathered}
\frac{\partial \bar{u}_{r}}{\partial t}+\bar{u}_{r} \frac{\partial \bar{u}_{r}}{\partial r}+\left[\frac{1}{r^{2}} \frac{\partial}{\partial r}\left(r^{2} R_{r r}\right)-\frac{R_{\theta \theta}+R_{\phi \phi}}{r}\right] \\
=-\frac{\partial \bar{p}}{\partial r}+2\left[\frac{1}{r^{2}} \frac{\partial}{\partial r}\left(r^{2} \bar{v} \frac{\partial \bar{u}_{r}}{\partial r}\right)-\frac{2 \bar{v} \bar{u}_{r}}{r^{2}}\right]-2\left[\frac{1}{r^{2}} \frac{\partial}{\partial r}\left(r^{2} M_{r r}\right)-\frac{M_{\theta \theta}+M_{\phi \phi}}{r}\right]
\end{gathered}
$$

The mean viscous diffusion vanishes because $B(t)$ is only a function of time. Note the presence of angular components for the tensors $R_{i j}$ and $M_{i j}$, again emphasizing that the fluctuating velocities and variations are fully three-dimensional.

\subsection{Individual Components of the Reynolds Stress Transport Equation}

The Reyrolds stress transport equation yields an equation for each of the diagonal components in spherical coordinates

$$
\begin{gathered}
\frac{\partial R_{r r}}{\partial t}+2 R_{r r} \frac{\partial \bar{u}_{r}}{\partial r}+\bar{u}_{r} \frac{\partial R_{r r}}{\partial r}=\frac{1}{r^{2}} \frac{\partial}{\partial r}\left(r^{2} 2 C_{D} s \sqrt{K} \frac{\partial R_{r r}}{\partial r}\right) \\
-4 C_{D} s \sqrt{K} \frac{\left(2 R_{r r}-R_{\theta \theta}-R_{\phi \phi}\right)}{r^{2}}+C_{B}\left[2 R_{r r} \frac{\partial \bar{u}_{r}}{\partial r}-\frac{2}{3}\left(R_{r r} \frac{\partial \bar{u}_{r}}{\partial r}+R_{\theta \theta} \frac{\bar{u}_{r}}{r}+R_{\phi \phi} \frac{\bar{u}_{r}}{r}\right)\right] \\
+\left(C_{R} \frac{\sqrt{K}}{s}+C_{H R} \sqrt{\left.\left(\frac{\partial \bar{u}_{r}}{\partial r}\right)^{2}+\frac{2 \bar{u}_{r}^{2}}{r^{2}}\right)\left(\frac{2}{3} K-R_{r r}\right)}\right. \\
+4 M_{r r} \frac{\partial \bar{u}_{r}}{\partial r}+\bar{v}\left[\frac{1}{r^{2}} \frac{\partial}{\partial r}\left(r^{2} \frac{\partial R_{r r}}{\partial r}\right)-2 \frac{\left(2 R_{r r}-R_{\theta \theta}-R_{\phi \phi}\right)}{r^{2}}\right]-(2 \bar{v}+Q) \frac{\Delta R_{r r}}{s^{2}}
\end{gathered}
$$




$$
\begin{gathered}
\frac{\partial R_{\theta \theta}}{\partial t}+2 R_{\theta \theta} \frac{\bar{u}_{r}}{r}+\bar{u}_{r} \frac{\partial R_{\theta \theta}}{\partial r}=\frac{1}{r^{2}} \frac{\partial}{\partial r}\left(r^{2} 2 C_{D} s \sqrt{K} \frac{\partial R_{\theta \theta}}{\partial r}\right) \\
+4 C_{D} s \sqrt{K} \frac{\left(R_{r r}-R_{\theta \theta}\right)}{r^{2}}+C_{B}\left[2 R_{\theta \theta} \frac{\bar{u}_{r}}{r}-\frac{2}{3}\left(R_{r r} \frac{\partial \bar{u}_{r}}{\partial r}+R_{\theta \theta} \frac{\bar{u}_{r}}{r}+R_{\phi \phi} \frac{\bar{u}_{r}}{r}\right)\right] \\
+\left(C_{R} \frac{\sqrt{K}}{s}+C_{H R} \sqrt{\left.\left(\frac{\partial \bar{u}_{r}}{\partial r}\right)^{2}+\frac{2 \bar{u}_{r}^{2}}{r^{2}}\right)\left(\frac{2}{3} K-R_{\theta \theta}\right)}\right. \\
+4 M_{\theta \theta} \frac{\bar{u}_{r}}{r}+\bar{v}\left[\frac{1}{r^{2}} \frac{\partial}{\partial r}\left(r^{2} \frac{\partial R_{\theta \theta}}{\partial r}\right)+2 \frac{\left(R_{r r}-R_{\theta \theta}\right)}{r^{2}}\right]-(2 \bar{v}+Q) \frac{\Delta R_{\theta \theta}}{s^{2}} \\
+4 C_{\phi} s \sqrt{K} \frac{\left(R_{r r}-R_{\phi \phi}\right)}{r^{2}}+C_{B}\left[2 R_{\phi \phi} \frac{\bar{u}_{r}}{r}-\frac{2}{3}\left(R_{r r} \frac{\partial \bar{u}_{r}}{\partial r}+R_{\theta \theta} \frac{\bar{u}_{r}}{r}+R_{\phi \phi} \frac{\bar{u}_{r}}{r}\right)\right] \\
+\left(C_{R} \frac{\sqrt{K}}{s}+C_{H R} \sqrt{\left.\left(\frac{\partial \bar{u}_{r}}{\partial r}\right)^{2}+\frac{2 \bar{u}_{r}^{2}}{r^{2}}\right)\left(\frac{2}{3} K-R_{\phi \phi}\right)}\right. \\
+4 M_{\phi \phi} \frac{\bar{u}_{r}}{r}+\bar{v}\left[\frac{1}{r^{2}} \frac{\partial}{\partial r}\left(r^{2} \frac{\partial R_{\phi \phi}}{\partial r}\right)+2 \frac{\left(R_{r r}-R_{\phi \phi}\right)}{r^{2}}\right]-(2 \bar{v}+Q) \frac{\Delta R_{\phi \phi}}{s^{2}}
\end{gathered}
$$

\subsection{Individual Components of the $M_{i j}$ Transport Equation}

The $M_{i j}$ transport equation yields an equation for each of the diagonal components in spherical coordinates

$$
\begin{gathered}
\frac{\partial M_{r r}}{\partial t}+2 M_{r r} \frac{\partial \bar{u}_{r}}{\partial r}-\frac{2}{3}\left(M_{r r} \frac{\partial \bar{u}_{r}}{\partial r}+M_{\theta \theta} \frac{\bar{u}_{r}}{r}+M_{\phi \phi} \frac{\bar{u}_{r}}{r}\right)+\bar{u}_{r} \frac{\partial M_{r r}}{\partial r} \\
-\frac{1}{r^{2}} \frac{\partial}{\partial r}\left(r^{2} 2 C_{H} s \sqrt{K} \frac{\partial M_{r r}}{\partial r}\right)+4 C_{H} s \sqrt{K} \frac{\left(2 M_{r r}-M_{\theta \theta}-M_{\phi \phi}\right)}{r^{2}} \\
=-\left[(2 \bar{v}+Q) \frac{\Delta}{s^{2}}+C_{M} \frac{\sqrt{K}}{s}+C_{H R} \sqrt{\left.\left(\frac{\partial \bar{u}_{r}}{\partial r}\right)^{2}+\frac{2 \bar{u}_{r}^{2}}{r^{2}}\right] M_{r r}}\right. \\
+2 Z_{r r} \frac{\partial \bar{u}_{r}}{\partial r}-\frac{2}{3}\left(Z_{r r} \frac{\partial \bar{u}_{r}}{\partial r}+Z_{\theta \theta} \frac{\bar{u}_{r}}{r}+Z_{\phi \phi} \frac{\bar{u}_{r}}{r}\right) \\
+\bar{v}\left[\frac{1}{r^{2}} \frac{\partial}{\partial r}\left(r^{2} \frac{\partial M_{r r}}{\partial r}\right)-2 \frac{\left(2 M_{r r}-M_{\theta \theta}-M_{\phi \phi}\right)}{r^{2}}\right]
\end{gathered}
$$




$$
\begin{gathered}
\frac{\partial M_{\theta \theta}}{\partial t}+2 M_{\theta \theta} \frac{\bar{u}_{r}}{r}-\frac{2}{3}\left(M_{r r} \frac{\partial \bar{u}_{r}}{\partial r}+M_{\theta \theta} \frac{\bar{u}_{r}}{r}+M_{\phi \phi} \frac{\bar{u}_{r}}{r}\right)+\bar{u}_{r} \frac{\partial M_{\theta \theta}}{\partial r} \\
-\frac{1}{r^{2}} \frac{\partial}{\partial r}\left(r^{2} 2 C_{H} s \sqrt{K} \frac{\partial M_{\theta \theta}}{\partial r}\right)-4 C_{H} s \sqrt{K} \frac{\left(M_{r r}-M_{\theta \theta}\right)}{r^{2}} \\
=-\left[(2 \bar{v}+Q) \frac{\Delta}{s^{2}}+C_{M} \frac{\sqrt{K}}{s}+C_{H R} \sqrt{\left.\left(\frac{\partial \bar{u}_{r}}{\partial r}\right)^{2}+\frac{2 \bar{u}_{r}^{2}}{r^{2}}\right] M_{\theta \theta}}\right. \\
+2 Z_{\theta \theta} \frac{\bar{u}_{r}}{r}-\frac{2}{3}\left(Z_{r r} \frac{\partial \bar{u}_{r}}{\partial r}+Z_{\theta \theta} \frac{\bar{u}_{r}}{r}+Z_{\phi \phi} \frac{\bar{u}_{r}}{r}\right) \\
+\bar{v}\left[\frac{1}{r^{2}} \frac{\partial}{\partial r}\left(r^{2} \frac{\partial M_{\theta \theta}}{\partial r}\right)+2 \frac{\left(M_{r r}-M_{\theta \theta}\right)}{r^{2}}\right]
\end{gathered}
$$

$$
\begin{gathered}
\frac{\partial M_{\phi \phi}}{\partial t}+2 M_{\phi \phi} \frac{\bar{u}_{r}}{r}-\frac{2}{3}\left(M_{r r} \frac{\partial \bar{u}_{r}}{\partial r}+M_{\theta \theta} \frac{\bar{u}_{r}}{r}+M_{\phi \phi} \frac{\bar{u}_{r}}{r}\right)+\bar{u}_{r} \frac{\partial M_{\phi \phi}}{\partial r} \\
-\frac{1}{r^{2}} \frac{\partial}{\partial r}\left(r^{2} 2 C_{H} s \sqrt{K} \frac{\partial M_{\phi \phi}}{\partial r}\right)-4 C_{H} s \sqrt{K} \frac{\left(M_{r r}-M_{\phi \phi}\right)}{r^{2}} \\
=-\left[(2 \bar{v}+Q) \frac{\Delta}{s^{2}}+C_{M} \frac{\sqrt{K}}{s}+C_{H R} \sqrt{\left.\left(\frac{\partial \bar{u}_{r}}{\partial r}\right)^{2}+\frac{2 \bar{u}_{r}^{2}}{r^{2}}\right] M_{\phi \phi}}\right. \\
+2 Z_{\phi \phi} \frac{\bar{u}_{r}}{r}-\frac{2}{3}\left(Z_{r r} \frac{\partial \bar{u}_{r}}{\partial r}+Z_{\theta \theta} \frac{\bar{u}_{r}}{r}+Z_{\phi \phi} \frac{\bar{u}_{r}}{r}\right) \\
+\bar{v}\left[\frac{1}{r^{2}} \frac{\partial}{\partial r}\left(r^{2} \frac{\partial M_{\phi \phi}}{\partial r}\right)+2 \frac{\left(M_{r r}-M_{\phi \phi}\right)}{r^{2}}\right]
\end{gathered}
$$




\subsection{Individual Components of the $Z_{i j}$ Transport Equation}

The $Z_{i j}$ tensor equation yields an equation for each of the diagonal components in spherical coordinates

$$
\begin{aligned}
& \frac{\partial Z_{r r}}{\partial t}+2 Z_{r r} \frac{\partial \bar{u}_{r}}{\partial r}-\frac{2}{3}\left(Z_{r r} \frac{\partial \bar{u}_{r}}{\partial r}+Z_{\theta \theta} \frac{\bar{u}_{r}}{r}+Z_{\phi \phi} \frac{\bar{u}_{r}}{r}\right)+\bar{u}_{r} \frac{\partial Z_{r r}}{\partial r} \\
& =\left[C_{Z} \frac{\sqrt{K}}{s}+C_{H R} \sqrt{\left(\frac{\partial \bar{u}_{r}}{\partial r}\right)^{2}+\frac{2 \bar{u}_{r}^{2}}{r^{2}}}\right]\left[\frac{1}{3}\left(Z_{r r}+Z_{\theta \theta}+Z_{\phi \phi}\right)-Z_{r r}\right] \\
& +\frac{1}{r^{2}} \frac{\partial}{\partial r}\left(r^{2} 2 C_{X} s \sqrt{K} \frac{\partial Z_{r r}}{\partial r}\right)-4 C_{D} s \sqrt{K} \frac{\left(2 Z_{r r}-Z_{\theta \theta}-Z_{\phi \phi}\right)}{r^{2}} \\
& -\frac{1}{3} \frac{1}{r^{2}} \frac{\partial}{\partial r}\left(r^{2} 2 C_{X} s \sqrt{K} \frac{\partial\left(Z_{r r}+Z_{\theta \theta}+Z_{\phi \phi}\right)}{\partial r}\right) \\
& \frac{\partial Z_{\theta \theta}}{\partial t}+2 Z_{\theta \theta} \frac{\bar{u}_{r}}{r}-\frac{2}{3}\left(Z_{r r} \frac{\partial \bar{u}_{r}}{\partial r}+Z_{\theta \theta} \frac{\bar{u}_{r}}{r}+Z_{\phi \phi} \frac{\bar{u}_{r}}{r}\right)+\bar{u}_{r} \frac{\partial Z_{\theta \theta}}{\partial r} \\
& =\left[C_{Z} \frac{\sqrt{K}}{s}+C_{H R} \sqrt{\left(\frac{\partial \bar{u}_{r}}{\partial r}\right)^{2}+\frac{2 \bar{u}_{r}^{2}}{r^{2}}}\right]\left[\frac{1}{3}\left(Z_{r r}+Z_{\theta \theta}+Z_{\phi \phi}\right)-Z_{\theta \theta}\right] \\
& +\frac{1}{r^{2}} \frac{\partial}{\partial r}\left(r^{2} 2 C_{X} s \sqrt{K} \frac{\partial Z_{\theta \theta}}{\partial r}\right)+4 C_{D} s \sqrt{K} \frac{\left(Z_{r r}-Z_{\theta \theta}\right)}{r^{2}} \\
& -\frac{1}{3} \frac{1}{r^{2}} \frac{\partial}{\partial r}\left(r^{2} 2 C_{X} s \sqrt{K} \frac{\partial\left(Z_{r r}+Z_{\theta \theta}+Z_{\phi \phi}\right)}{\partial r}\right) \\
& \frac{\partial Z_{\phi \phi}}{\partial t}+2 Z_{\phi \phi} \frac{\bar{u}_{r}}{r}-\frac{2}{3}\left(Z_{r r} \frac{\partial \bar{u}_{r}}{\partial r}+Z_{\theta \theta} \frac{\bar{u}_{r}}{r}+Z_{\phi \phi} \frac{\bar{u}_{r}}{r}\right)+\bar{u}_{r} \frac{\partial Z_{\phi \phi}}{\partial r} \\
& =\left[C_{Z} \frac{\sqrt{K}}{s}+C_{H R} \sqrt{\left(\frac{\partial \bar{u}_{r}}{\partial r}\right)^{2}+\frac{2 \bar{u}_{r}^{2}}{r^{2}}}\right]\left[\frac{1}{3}\left(Z_{r r}+Z_{\theta \theta}+Z_{\phi \phi}\right)-Z_{\phi \phi}\right] \\
& +\frac{1}{r^{2}} \frac{\partial}{\partial r}\left(r^{2} 2 C_{X} s \sqrt{K} \frac{\partial Z_{\phi \phi}}{\partial r}\right)+4 C_{D} s \sqrt{K} \frac{\left(Z_{r r}-Z_{\phi \phi}\right)}{r^{2}} \\
& -\frac{1}{3} \frac{1}{r^{2}} \frac{\partial}{\partial r}\left(r^{2} 2 C_{X} s \sqrt{K} \frac{\partial\left(Z_{r r}+Z_{\theta \theta}+Z_{\phi \phi}\right)}{\partial r}\right)
\end{aligned}
$$




\subsection{Mean Internal Energy, Mean Kinetic Energy, and the Turbulent Kinetic Energy}

The mean internal energy, mean kinetic energy, and the turbulent kinetic energy equations are all scalar equations, so that for each variable there is only one equation. The mean internal energy equation in spherical coordinates becomes

$$
\begin{gathered}
\frac{\partial \bar{e}}{\partial t}+\bar{u}_{r} \frac{\partial \bar{e}}{\partial r}=2 \bar{v}\left[\left(\frac{\partial \bar{u}_{r}}{\partial r}\right)^{2}+\frac{2 \bar{u}_{r}^{2}}{r^{2}}\right]+(2 \bar{v}+Q) \frac{\Delta 2 K}{s^{2}} \\
+\frac{\bar{v}}{r^{2}} \frac{\partial}{\partial r}\left[r^{2}\left(\frac{1}{r^{2}} \frac{\partial\left(r^{2} R_{r r}\right)}{\partial r}-\frac{\left(R_{\theta \theta}+R_{\phi \phi}\right)}{r}\right)\right]-4\left(M_{r r} \frac{\partial \bar{u}_{r}}{\partial r}+M_{\theta \theta} \frac{\bar{u}_{r}}{r}+M_{\phi \phi} \frac{\bar{u}_{r}}{r}\right)
\end{gathered}
$$

The mean kinetic energy equation in spherical coordinates becomes

$$
\begin{gathered}
\frac{\partial K E}{\partial t}+\bar{u}_{r} \frac{\partial K E}{\partial r}+\bar{u}_{r}\left[\frac{1}{r^{2}} \frac{\partial}{\partial r}\left(r^{2} R_{r r}\right)-\frac{R_{\theta \theta}+R_{\phi \phi}}{r}\right] \\
=-\bar{u}_{r} \frac{\partial \bar{p}}{\partial r}+\bar{v} \frac{1}{r^{2}} \frac{\partial}{\partial r}\left(r^{2} \frac{\partial K E}{\partial r}\right) \\
-\bar{v}\left[\left(\frac{\partial \bar{u}_{r}}{\partial r}\right)^{2}+\frac{2 \bar{u}_{r}^{2}}{r^{2}}\right]-2 \bar{u}_{r}\left[\frac{1}{r^{2}} \frac{\partial}{\partial r}\left(r^{2} M_{r r}\right)-\frac{M_{\theta \theta}+M_{\phi \phi}}{r}\right]
\end{gathered}
$$

The turbulent kinetic energy equation in spherical coordinates becomes

$$
\begin{aligned}
& \frac{\partial K}{\partial t}+R_{r r} \frac{\partial \bar{u}_{r}}{\partial r}+R_{\theta \theta} \frac{\bar{u}_{r}}{r}+R_{\phi \phi} \frac{\bar{u}_{r}}{r}+\bar{u}_{r} \frac{\partial K}{\partial r}=\frac{1}{r^{2}} \frac{\partial}{\partial r}\left(r^{2} 2 C_{X} s \sqrt{K} \frac{\partial K}{\partial r}\right) \\
& +2 M_{r r} \frac{\partial \bar{u}_{r}}{\partial r}+2 M_{\theta \theta} \frac{\bar{u}_{r}}{r}+2 M_{\phi \phi} \frac{\bar{u}_{r}}{r}+\bar{v} \frac{1}{r^{2}} \frac{\partial}{\partial r}\left(r^{2} \frac{\partial K}{\partial r}\right)-(2 \bar{v}+Q) \frac{\Delta K}{s^{2}}
\end{aligned}
$$




\subsection{Summary of the Spherical Formulation}

In the spherical formulation the tensor equations $R_{i j}, M_{i j}$, and $Z_{i j}$ can no longer be expressed in the compact tensor notation, but instead as individual component equations. This must be done because the velocity gradients and the diffusion terms are different for the different tensor components. For each of the tensors there are three individual component equations, the $r r$-component, the $\theta \theta$-component, and the $\phi \phi$-component. The off diagonal components vanish since all of the mean quantities in the crenulative material are independent of angle, and there is no mean angular motion of the fluid. The trace of $R_{i j}, M_{i j}$, and $Z_{i j}$ is still expressed as the sum of the diagonals in spherical form

$$
\begin{gathered}
R_{l l}=R_{r r}+R_{\theta \theta}+R_{\phi \phi}=2 K \\
M_{l l}=M_{r r}+M_{\theta \theta}+M_{\phi \phi} \\
Z_{l l}=Z_{r r}+Z_{\theta \theta}+Z_{\phi \phi}
\end{gathered}
$$

The mean internal energy, mean kinetic energy, and turbulence energy equations remain single equations in their spherical form since they were scalar equations to begin with.

Now that the governing equations are in spherical form [14] we can analyze the properties of the equations in both Cartesian tensor form and in spherical form. Also, since the governing equations are in spherical form we can nondimensionalize the equations and the boundary conditions. 


\section{Chapter 7}

\section{Properties of the Modeled Governing Equations}

The modeled governing equations, for the restricted set of circumstances described in Chapters 5 and 6 display various properties that tend to validate the idea of a Reynolds decomposition approach for understanding crenulation.

\subsection{Primary Driver for Crenulative Turbulence}

The $Z_{i j}$ tensor describes the configuration of the nonhomogenities, and when coupled to the rate-of-strain tensor it produces a dominant contribution to the evolution of $M_{i j}$, i.e. a "first order response to crenulation".

$$
\frac{\partial M_{i j}}{\partial t}=Z_{j k}\left(\frac{\partial \bar{u}_{i}}{\partial x_{k}}+\frac{\partial \bar{u}_{k}}{\partial x_{i}}\right)+\ldots
$$

We sometimes call $M_{i j}$ the "first order response tensor". This tensor, when coupled to the rate-of-strain tensor, produces the dominant contribution to the evolution of Reynolds stress, i.e. a "second order response to crenulation".

$$
\frac{\partial R_{i j}}{\partial t}=M_{j l}\left(\frac{\partial \bar{u}_{i}}{\partial x_{l}}+\frac{\partial \bar{u}_{l}}{\partial x_{i}}\right)+M_{i l}\left(\frac{\partial \bar{u}_{j}}{\partial x_{l}}+\frac{\partial \bar{u}_{l}}{\partial x_{j}}\right)+\ldots
$$

It can be seen that $Z_{i j}$ contributes to the evolution of Reynolds stress in a two-step process. The effects of $Z_{i j}$ also contribute to the mean internal energy and the mean kinetic energy through $M_{i j}$.

Consider the case of a spherical shell of fluid initially at rest, with no $M_{i j}$ and no Reynolds stress. If the fluid is set into convergent motion, and there is a nonhomogeneity 
in the viscosity coefficient, the crenulative instability is initiated. The rate-of-strain tensor coupled to $Z_{i j}$ "drives" the crenulation. Because of this driving process we call the coupling of the rate-of-strain tensor to $Z_{i j}$ the "primary driver" of the crenulation

$$
Z_{j k}\left(\frac{\partial \bar{u}_{i}}{\partial x_{k}}+\frac{\partial \bar{u}_{k}}{\partial x_{i}}\right)
$$

The primary driver for crenulation is directly analogous to the primary driver for variable density turbulence [15]

$$
\overline{\rho^{\prime} \rho^{\prime}} \frac{d \bar{p}}{d x}
$$

In variable-density turbulence a mean pressure gradient coupled to the variations in density drives the turbulence. The pressure gradient accelerates the local regions of different density relative to each other, thus creating velocity fluctuations or turbulence. The "first order response" is a creation of net mass flux; in the "second order response" the mass flux couples to the mean pressure gradient to drive the evolution of Reynolds stress.

\subsection{The Limit as the Nonhomogeneities Vanish}

In the limit as the nonhomogeneities vanish we obtain a modeled set of governing equations for classical turbulence. As the nonhomogeneities become small the effect of the primary driver weakens (assuming the rate-of-strain does not approach infinity), therefore giving very weak first and second order responses to crenulation. This limit can be shown by letting $s \rightarrow \infty$ and/or $\overline{v^{\prime} v^{\prime}} \rightarrow 0$. Letting $s \rightarrow \infty$ means the separation distance between nonhomogeneities is very large. Letting $\overline{v^{\prime} v^{\prime}} \rightarrow 0$ means the viscous fluctuations vanish even though the separation distance between nonhomogeneities remains at the scale of $s$. 
In either case the fluid becomes homogeneous (no $Z_{i j}$ ) and the crenulation occurs at the atomic level, which is described by the homogenous viscous behavior.

The incompressibility condition remains unchanged

$$
\frac{\partial \bar{u}_{l}}{\partial x_{l}}=0
$$

The mean momentum looses the contribution from the first order response tensor

$$
\frac{\partial \bar{u}_{i}}{\partial t}+\frac{\partial \bar{u}_{i} \bar{u}_{l}}{\partial x_{l}}+\frac{\partial R_{i l}}{\partial x_{l}}=-\frac{\partial \bar{p}}{\partial x_{i}}+\frac{\partial}{\partial x_{l}}\left[\bar{v}\left(\frac{\partial \bar{u}_{i}}{\partial x_{l}}+\frac{\partial \bar{u}_{l}}{\partial x_{i}}\right)\right]
$$

The Reynolds stress tensor equation also loses the contribution from the first order response tensor, the term due to the collision of nonhomogeneities, and the enhancement to a return to isotropy.

$$
\begin{gathered}
\frac{\partial R_{i j}}{\partial t}+R_{j l} \frac{\partial \bar{u}_{i}}{\partial x_{l}}+R_{i l} \frac{\partial \bar{u}_{j}}{\partial x_{l}}+\bar{u}_{l} \frac{\partial R_{i j}}{\partial x_{l}}=\frac{\partial}{\partial x_{l}}\left[C_{D} \frac{s}{\sqrt{K}} R_{l m} \frac{\partial R_{i j}}{\partial x_{m}}\right] \\
+C_{B}\left(R_{i l} \frac{\partial \bar{u}_{j}}{\partial x_{l}}+R_{j l} \frac{\partial \bar{u}_{i}}{\partial x_{l}}-\frac{2}{3} R_{k l} \frac{\partial \bar{u}_{k}}{\partial x_{l}} \delta_{i j}\right)+\left(C_{R} \frac{\sqrt{K}}{s}\right)\left(\frac{2}{3} K \delta_{i j}-R_{i j}\right) \\
+\bar{v} \frac{\partial^{2} R_{i j}}{\partial x_{l}^{2}}-2 \bar{v} \frac{\Delta R_{i j}}{s^{2}}
\end{gathered}
$$

The scale $s$ is still the dominant scale of the turbulence, which is no longer related to the separation distance between nonhomogeneities, and must be determined by a careful investigation of the full turbulence spectrum [15]. This classical turbulence limit does not have a primary driver, so a small "seed" of turbulence in the domain is needed to initiate the growth of turbulence. Thus the limit of classical turbulence transport theory is contained in our formulation for crenulative turbulence. 


\subsection{A First Order Approximation for Behavior of $Z_{i j}$}

To derive a first order approximation for the evolution of $Z_{i j}$ we retain only the advection and mean flow coupling terms in the $Z_{i j}$ equation (5.14)

$$
\frac{\partial Z_{i j}}{\partial t}+\vec{u}_{l} \frac{\partial Z_{i j}}{\partial x_{l}}=-Z_{i l} \frac{\partial \bar{u}_{l}}{\partial x_{j}}-Z_{j l} \frac{\partial \bar{u}_{l}}{\partial x_{i}}+\frac{2}{3} Z_{n l} \frac{\partial \bar{u}_{l}}{\partial x_{n}}
$$

Let

$$
Z_{i j}=Z_{i j}^{o}+\xi_{i j}
$$

in which $Z_{i j}^{o}$ describes the initial state of the nonhomogeneities and $\xi_{i j}$ is a perturbation from that initial state. Then the $Z_{i j}$ equation becomes

$$
\frac{\partial \xi_{i j}}{\partial t}+\bar{u}_{l} \frac{\partial \xi_{i j}}{\partial x_{l}}=-Z_{i l}^{o} \frac{\partial \bar{u}_{l}}{\partial x_{j}}-Z_{j l}^{o} \frac{\partial \bar{u}_{l}}{\partial x_{i}}+\frac{2}{3} Z_{n l}^{o} \frac{\partial \bar{u}_{l}}{\partial x_{n}}
$$

Assuming a steady state circumstance and spherical form the diagonal components of equation (7.2) become

$$
\begin{aligned}
& \bar{u}_{r} \frac{\partial \xi_{r r}}{\partial r}=-2 Z_{r r}^{o} \frac{\partial \bar{u}_{r}}{\partial r}+\frac{2}{3}\left(Z_{r r}^{o} \frac{\partial \bar{u}_{r}}{\partial r}+Z_{\theta \theta}^{o} \frac{\bar{u}_{r}}{r}+Z_{\phi \phi}^{o} \frac{\bar{u}_{r}}{r}\right) \\
& \bar{u}_{r} \frac{\partial \xi_{\theta \theta}}{\partial r}=-2 Z_{\theta \theta}^{o} \frac{\bar{u}_{r}}{r}+\frac{2}{3}\left(Z_{r r}^{o} \frac{\partial \bar{u}_{r}}{\partial r}+Z_{\theta \theta}^{o} \frac{\bar{u}_{r}}{r}+Z_{\phi \phi}^{o} \frac{\bar{u}_{r}}{r}\right) \\
& \bar{u}_{r} \frac{\partial \xi_{\phi \phi}}{\partial r}=-2 Z_{\phi \phi}^{o} \frac{\bar{u}_{r}}{r}+\frac{2}{3}\left(Z_{r r}^{o} \frac{\partial \bar{u}_{r}}{\partial r}+Z_{\theta \theta}^{o} \frac{\bar{u}_{r}}{r}+Z_{\phi \phi}^{o} \frac{\bar{u}_{r}}{r}\right)
\end{aligned}
$$


Because of the incompressibility condition, $\bar{u}_{r}=\frac{B(t)}{r^{2}}$, for spherical convergence, equations (7.3) reduce to

$$
\begin{gathered}
\frac{\partial \xi_{r r}}{\partial r}=\frac{8}{3} \frac{Z_{r r}^{o}}{r}+\frac{2}{3} \frac{Z_{\theta \theta}^{o}}{r}+\frac{2}{3} \frac{Z_{\phi \phi}^{o}}{r} \\
\frac{\partial \xi_{\theta \theta}}{\partial r}=-\frac{4}{3} \frac{Z_{r r}^{o}}{r}-\frac{4}{3} \frac{Z_{\theta \theta}^{o}}{r}+\frac{2}{3} \frac{Z_{\phi \phi}^{o}}{r} \\
\frac{\partial \xi_{\phi \phi}}{\partial r}=-\frac{4}{3} \frac{Z_{r r}^{o}}{r}+\frac{2}{3} \frac{Z_{\theta \theta}^{o}}{r}-\frac{4}{3} \frac{Z_{\phi \phi}^{o}}{r}
\end{gathered}
$$

If $Z_{i j}$ is initially stochastically isotropic, $Z_{r r}^{o}=Z_{\theta \theta}^{o}=Z_{\phi \phi}^{o}=Z^{o}$, then equations (7.4) reduce to

$$
\begin{gathered}
\frac{\partial \xi_{r r}}{\partial r}=4 \frac{Z^{o}}{r} \\
\frac{\partial \xi_{\theta \theta}}{\partial r}=-2 \frac{Z^{o}}{r} \\
\frac{\partial \xi_{\phi \phi}}{\partial r}=-2 \frac{Z^{o}}{r}
\end{gathered}
$$

Applying the boundary condition $Z_{i j}\left(r=R_{2}\right)=Z^{o} \delta_{i j}$, where $R_{2}$ is the outer radius of the spherical shell of fluid, the diagonal components of $Z_{i j}$ can be solved directly 


$$
\begin{gathered}
Z_{r r}=Z_{r r}^{o}+4 Z_{r r}^{o} \ln \left(\frac{r}{R_{2}}\right) \\
Z_{\theta \theta}=Z_{\theta \theta}^{o}-2 Z_{\theta \theta}^{o} \ln \left(\frac{r}{R_{2}}\right) \\
Z_{\phi \phi}=Z_{\phi \phi}^{o}-2 Z_{\phi \phi}^{o} \ln \left(\frac{r}{R_{2}}\right)
\end{gathered}
$$

The behavior of the first order approximation for $Z_{i j}$ can be seen graphical in Fig. 7.1 as a function of radius in a steady-state circumstance.

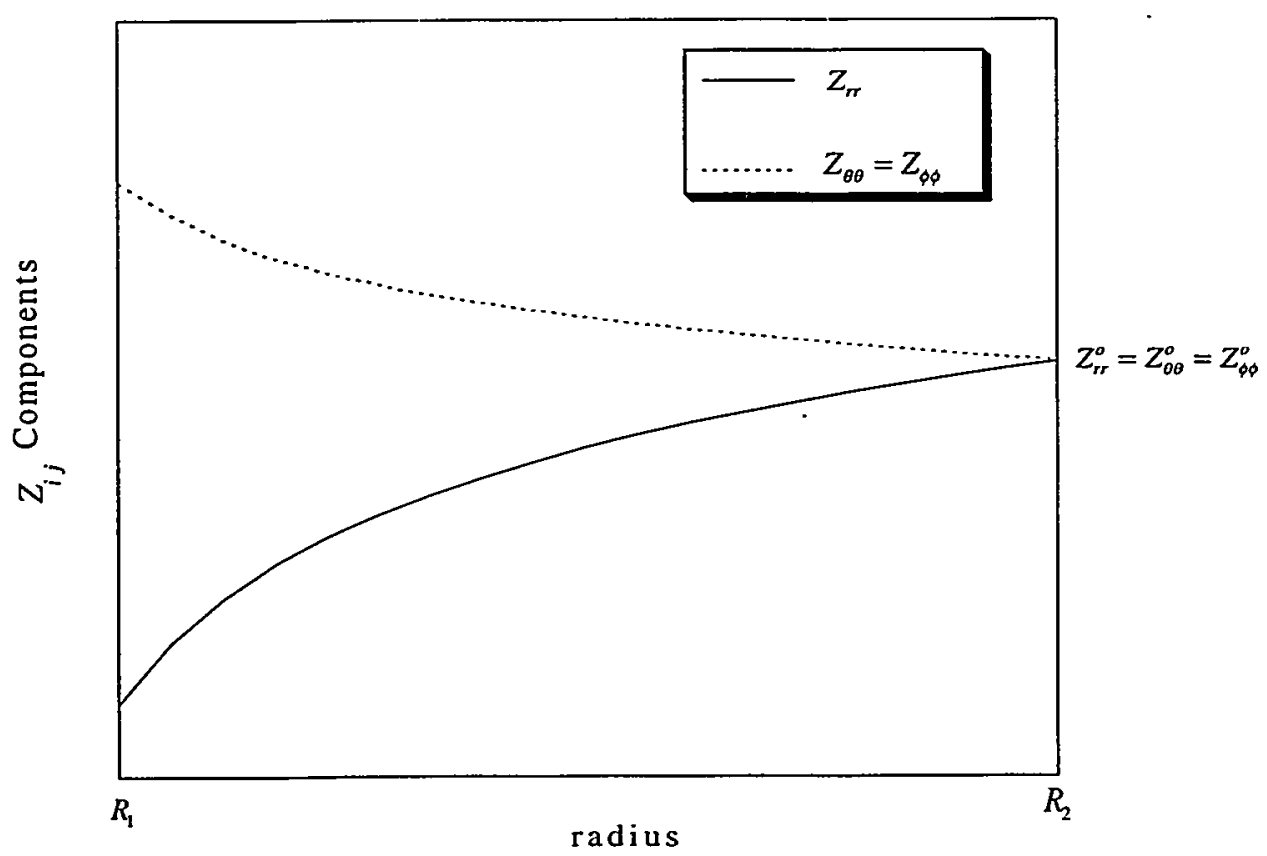

Figure 7.1: First Order Approximation for $Z_{i j}$ 
The interpretation of these results is discussed in Section 7.4, where it is shown that $Z_{r r} \leq Z_{\theta \theta}=Z_{\phi \phi}$ means the nonhomogeneities are elongating in the radial direction and contracting in the two angular directions as shown in Fig. 7.2.

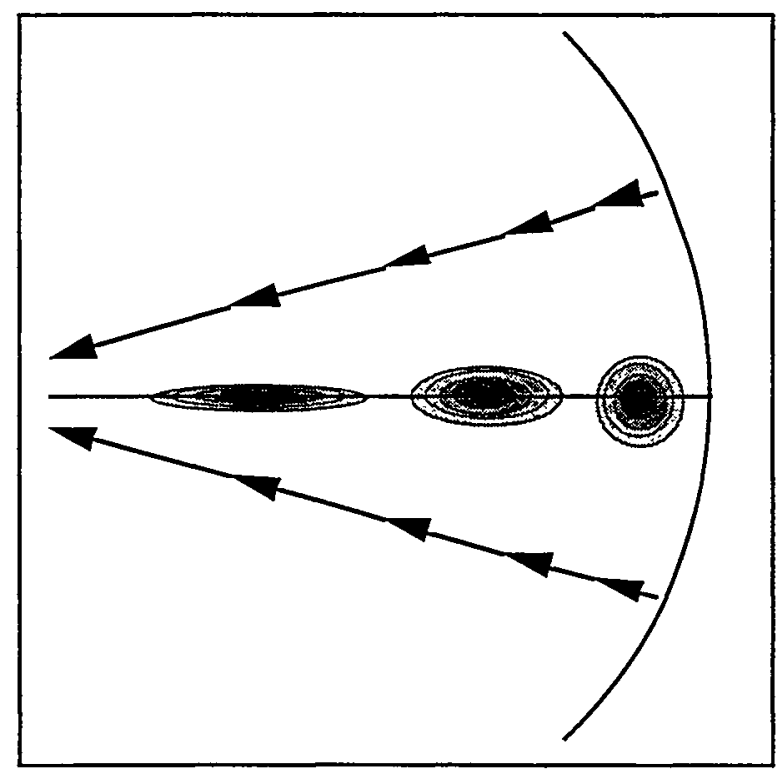

Figure 7.2: Elongation of the Nonhomogeneities During Spherical Convergence

Equations (7.6) are very good approximations for the diagonal components of $Z_{i j}$ near the initial isotropic state, which is near the outer radius of the spherical shell. Linearization of equation (7.1) about the initial isotropic state at the outer radius results in bad approximations for $Z_{i j}$ near the inner radius. In general bad results away from the point of linearization is expected. 


\subsection{Cosine Distribution of the Instantaneous Kinematic Viscosity}

A cosine distribution of kinematic viscosity in the three orthogonal directions in a

Cartesian coordinate system,

$$
v=\bar{v}+\frac{\delta v}{2} \cos \left(\frac{2 \pi x}{s_{x}}\right) \cos \left(\frac{2 \pi y}{s_{y}}\right) \cos \left(\frac{2 \pi z}{s_{z}}\right)
$$

can be used as a basis for interpreting the significance of nonisotropy of $Z_{i j}$. In this qualitative model, $\delta v$ is the difference between the maximum and minimum values of the instantaneous kinematic viscosity, $s_{x}$ is the scale of the nonhomogeneities in the $x$ direction, $s_{y}$ and $s_{z}$ are the scales of the nonhomogeneities in the other two orthogonal directions. If $s=s_{x}=s_{y}=s_{z}$, then $Z_{i j}$ is isotropic. From the fluctuating component of the instantaneous kinematic viscosity,

$$
v^{\prime}=\frac{\delta v}{2} \cos \left(\frac{2 \pi x}{s_{x}}\right) \cos \left(\frac{2 \pi y}{s_{y}}\right) \cos \left(\frac{2 \pi z}{s_{z}}\right)
$$

we can derive the magnitudes of $Z_{x x}, Z_{y y}$, and $Z_{z z}$, as defined by $Z_{i j}=\frac{\overline{\partial v^{\prime}}}{\partial x_{i}} \frac{\partial v^{\prime}}{\partial x_{j}}$. With the cosine representation, the ensemble average is obtained by integrating over a unit quadrilateral.

$$
\begin{aligned}
& Z_{x x}=\left(\frac{\pi \delta v}{s_{x}}\right)^{2} \frac{1}{s_{x} s_{y} s_{z}} \int_{-s_{z} / 2}^{+s_{z} / 2} \int_{-s_{y} / 2}^{+s_{y} / 2} \int_{-s_{x} / 2}^{+s_{x} / 2} \sin ^{2}\left(\frac{2 \pi x}{s_{x}}\right) \cos ^{2}\left(\frac{2 \pi y}{s_{y}}\right) \cos ^{2}\left(\frac{2 \pi z}{s_{z}}\right) d x d y d z \\
& Z_{y y}=\left(\frac{\pi \delta v}{s_{y}}\right)^{2} \frac{1}{s_{x} s_{y} s_{z}} \int_{-s_{z} / 2}^{+s_{z} / 2} \int_{-s_{y} / 2}^{+s_{y} / 2} \int_{-s_{x} / 2}^{+s_{x} / 2} \cos ^{2}\left(\frac{2 \pi x}{s_{x}}\right) \sin ^{2}\left(\frac{2 \pi y}{s_{y}}\right) \cos ^{2}\left(\frac{2 \pi z}{s_{z}}\right) d x d y d z
\end{aligned}
$$




$$
\begin{aligned}
& Z_{z z}=\left(\frac{\pi \delta v}{s_{z}}\right)^{2} \frac{1}{s_{x} s_{y} s_{z}} \int_{-s_{z} / 2}^{+s_{z} / 2} \int_{-s_{y} / 2}^{+s_{y} / 2} \int_{-s_{x} / 2}^{+s_{x} / 2} \cos ^{2}\left(\frac{2 \pi x}{s_{x}}\right) \cos ^{2}\left(\frac{2 \pi y}{s_{y}}\right) \sin ^{2}\left(\frac{2 \pi z}{s_{z}}\right) d x d y d z \\
& \text { or } \\
& Z_{x x}=\frac{\pi^{2}}{8}\left(\frac{\delta v}{s_{x}}\right)^{2} \\
& Z_{y y}=\frac{\pi^{2}}{8}\left(\frac{\delta v}{s_{y}}\right)^{2} \\
& Z_{z z}=\frac{\pi^{2}}{8}\left(\frac{\delta v}{s_{z}}\right)^{2}
\end{aligned}
$$

If the scale of the nonhomogeneities in the $x$ direction, $s_{x}$, is greater than the two scales in the other two orthogonal directions, e.g. $s_{x} \geq s_{y}=s_{z}$, then the elongation of the nonhomogeneities is occurring in the $x$ direction. This, then, confirms the interpretation in Section 7.3, regarding the radial elongation of the converging entities.

In the modeling of $Z_{i j}$ transport, discussed in Chapter 5, we proposed the contraction of $Z_{i j}$ to be a constant, i.e. $Z_{n n}=$ constant. The contraction of $Z_{i j}$ with the cosine distribution is

$$
Z_{n n}=Z_{x x}+Z_{y y}+Z_{z z}=\frac{\pi^{2}}{8}\left(\frac{\delta v}{s_{x}}\right)^{2}+\frac{\pi^{2}}{8}\left(\frac{\delta v}{s_{y}}\right)^{2}+\frac{\pi^{2}}{8}\left(\frac{\delta v}{s_{z}}\right)^{2}=\text { constant }
$$

To remain a constant while $s_{x}$ increases, the two other scales, $s_{y}$ and $s_{z}$, must decrease. This means elongation of a nonhomogeneity in one direction implies the contraction of the nonhomogeneity in the other two directions. Assuming $s_{y}=s_{z}$, and that $s_{x}$ elongates by a factor $f_{x}$, then the other two scales contract by a factor $f_{y}=f_{z}$ given by 


$$
f_{y}=f_{z}=\sqrt{\frac{2 f_{x}^{2}}{3 f_{x}^{2}-1}}
$$

We can now use the results from the first order approximation for $Z_{i j}$ and the results from the cosine distribution to see how the scales of the nonhomogeneities change with the radius of the spherical shell of fluid in a steady state circumstance. Equating equations (7.6) to equations (7.7) in spherical form

$$
\begin{gathered}
Z_{r r}=Z_{r r}^{o}+4 Z_{r r}^{o} \ln \left(\frac{r}{R_{2}}\right)=\frac{\pi^{2}}{8}\left(\frac{\delta v}{s_{r}}\right)^{2} \\
Z_{\theta \theta}=Z_{\theta \theta}^{o}-2 Z_{\theta \theta}^{o} \ln \left(\frac{r}{R_{2}}\right)=\frac{\pi^{2}}{8}\left(\frac{\delta v}{s_{\theta}}\right)^{2} \\
Z_{\phi \phi}=Z_{\phi \phi}^{o}-2 Z_{\phi \phi}^{o} \ln \left(\frac{r}{R_{2}}\right)=\frac{\pi^{2}}{8}\left(\frac{\delta v}{s_{\phi}}\right)^{2}
\end{gathered}
$$

At the initial state the diagonal components of $Z_{i j}$ are equal (the nonhomogeneities are isotropic) and are characterized by the initial scale $s_{o}$

$$
Z_{r r}^{o}=Z_{\theta \theta}^{o}=Z_{\phi \phi}^{o}=\frac{\pi^{2}}{8}\left(\frac{\delta v}{s_{o}}\right)^{2}
$$

Using this in combination with equations (7.8) the individual scales as functions of the radius are given by

$$
1+4 \ln \left(\frac{r}{R_{2}}\right)=\left(\frac{s_{o}}{s_{r}}\right)^{2}
$$




$$
\begin{aligned}
& 1-2 \ln \left(\frac{r}{R_{2}}\right)=\left(\frac{s_{o}}{s_{\theta}}\right)^{2} \\
& 1-2 \ln \left(\frac{r}{R_{2}}\right)=\left(\frac{s_{o}}{s_{\phi}}\right)^{2}
\end{aligned}
$$

Equations (7.10) are good approximations for how the scales change as a function of radius near the outer radius of the spherical shell of fluid. There is some question as to whether the volume of the nonhomogeneities is conserved while being elongated. With the cosine analysis, we can investigate this condition as long as the departures from the cosine form are small. As each nonhomogeneity converges, its form becomes more egg shaped, and the measure of its volume departs from the value $s_{r} s_{\theta} s_{\phi}$ that is appropriate near the outer edge. To show the volume of the nonhomogeneities is conserved to first order, we write

$$
r=R_{2}(1-\omega)
$$

where $\omega<<1$. Then equations (7.10) can be rewritten as

$$
\begin{aligned}
& 1+4 \ln (1-\omega)=\left(\frac{s_{o}}{s_{r}}\right)^{2} \\
& 1-2 \ln (1-\omega)=\left(\frac{s_{o}}{s_{\theta}}\right)^{2} \\
& 1-2 \ln (1-\omega)=\left(\frac{s_{o}}{s_{\phi}}\right)^{2}
\end{aligned}
$$


Using a series expansion for the natural logarithm and keeping terms of order $\omega$, equations (7.11) become

$$
\begin{aligned}
& 1-4 \omega=\left(\frac{s_{o}}{s_{r}}\right)^{2} \\
& 1+2 \omega=\left(\frac{s_{o}}{s_{\theta}}\right)^{2} \\
& 1+2 \omega=\left(\frac{s_{o}}{s_{\phi}}\right)^{2}
\end{aligned}
$$

Solving for the ratio of the elongated volume to the initial isotropic volume of the nonhomogeneities to order $\omega$

$$
\frac{s_{r} s_{\theta} s_{\phi}}{s_{o}^{3}}=\sqrt{\frac{1}{(1-4 \omega)(1+2 \omega)^{2}}}=\sqrt{\frac{1}{1-12 \omega^{2}-16 \omega^{3}}} \approx 1+O\left(\omega^{2}\right)
$$

Because the ratio is unity, the volume of the elongating nonhomogeneities is conserved until its distortion from the cosine form is so great as to make this measure of volume inappropriate. 


\subsection{Nonuniform Distribution of the Instantaneous Kinematic Viscosity}

A nonuniform distribution of kinematic viscosity in a three dimensional spherical coordinate system can be used as a basis for further understanding of $Z_{i j}$. Consider the distribution along the radial direction as seen in Fig. 7.3.

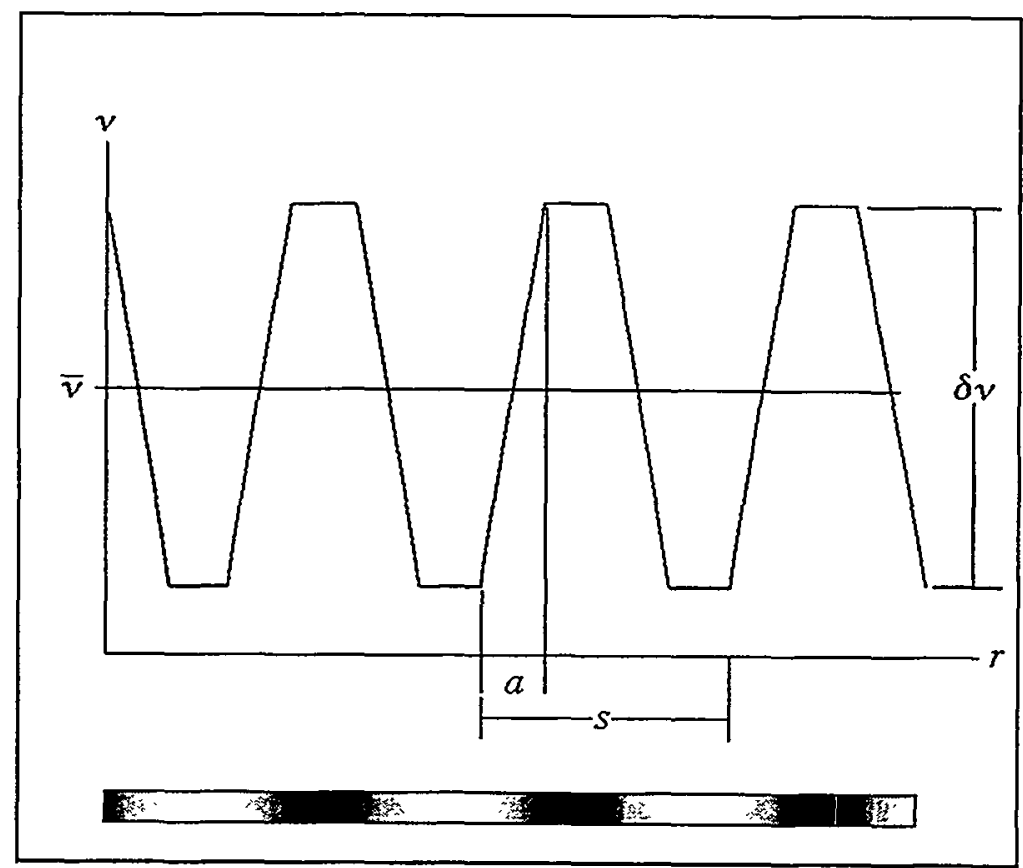

Figure 7.3: Nonuniform Distribution of Kinematic Viscosity in Radial Direction

In this distribution the spherical nonhomogeneities have high constant viscosity centers with a variable-viscosity skin of thickness $a$, surrounded by a low constant viscosity fluid. The high-viscosity centers are represented by the black regions, the skin of variable viscosity is represented with the gray gradient, and the low viscosity fluid is represented by the white areas, as seen in Fig. 7.3 and in the unit cell in Fig. 7.4. 


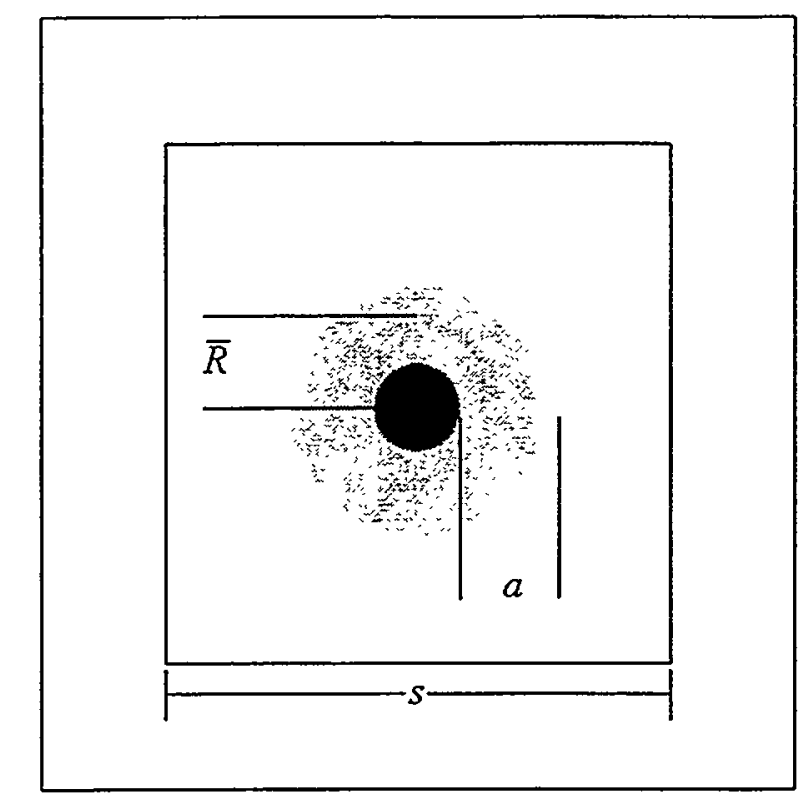

Figure 7.4: Unit Cell of the Nonuniform Distribution

To calculate $Z_{r r}$ we must integrate about a unit cell of size $s^{3}$

$$
Z_{r r}=\frac{1}{s^{3}} \iiint_{V o l}\left(\frac{\partial v^{\prime}}{\partial r}\right)^{2} d V
$$

Because the slope of the region of variable viscosity is constant in the radial direction and there is no contribution from the high-constant-viscosity centers or low-constant-viscosity fluid, $Z_{r r}$ is

$$
Z_{r r}=\frac{1}{s^{3}}\left(\frac{\partial v^{\prime}}{\partial r}\right)^{2} \iiint_{V o l} d V=\frac{1}{s^{3}}\left(\frac{\delta v}{a}\right)^{2} \frac{4 \pi}{3}\left(R_{o}^{3}-R_{i}^{3}\right)
$$

where $R_{i}$ is the radius to the inner part of the skin and $R_{o}$ is the radius to the outer part of the skin. The inner and outer radii can be expressed in terms of the nominal radius of the skin, $\bar{R}$, and the skin thickness, $a$, 


$$
R_{i}=\bar{R}-\frac{a}{2} \quad R_{o}=\bar{R}+\frac{a}{2}
$$

so that

$$
Z_{r r}=\frac{1}{s^{3}}\left(\frac{\delta v}{a}\right)^{2} \frac{4 \pi}{3}\left[6 \bar{R}^{2}\left(\frac{a}{2}\right)+2\left(\frac{a}{2}\right)^{3}\right]
$$

If the skin thickness is much smaller than the nominal radius, $a<<\bar{R}$, then $Z_{r r}$ can be approximated by the following

$$
\begin{gathered}
Z_{r r} \approx \frac{1}{s^{3}}\left(\frac{\delta v}{a}\right)^{2} 4 \pi \bar{R}^{2} a \\
Z_{r r} \approx \frac{4 \pi \bar{R}^{2}}{s a}\left(\frac{\delta v}{s}\right)^{2}
\end{gathered}
$$

Since $Z_{i j}$ is initially isotropic then

$$
Z_{r r}=Z_{\theta \theta}=Z_{\phi \phi} \approx \frac{4 \pi \bar{R}^{2}}{s a}\left(\frac{\delta v}{s}\right)^{2}
$$

This result has very significant implications, especially in comparison to equation (7.9). As the nominal radius of the nonhomogeneities increases the degree of nonhomogeneity in the system also increases; as the skin thickness decreases the degree of nonhomogeneity also increases; and as the separation scale of the nonhomogeneities decreases the degree of nonhomogeneity increases. It is of particular significance that decreasing skin thickness enhances the measure, $Z_{i j}$, of crenulative potential, with implications that are likely to be especially important for understanding the behavior of metal grains with narrow regions of plastic flow subject to intense distortive stresses. Equation (7.12) is important for 
choosing the nondimensional value of $Z_{i j}$ at the outer boundary (outer radius of the spherical shell) for the examples described in Chapter 8.

\subsection{Positive Definite Entropy Changes}

The instantaneous internal energy equation (2.12) governs how the mechanical energy dissipates to heat for an incompressible fluid. The dissipation function must always work to increase the internal energy for an incompressible fluid in an irreversible fashion.

$$
\frac{\partial e}{\partial t}+u_{l} \frac{\partial e}{\partial x_{l}}=\frac{D e}{D t}=v\left(\frac{\partial u_{i}}{\partial x_{k}}+\frac{\partial u_{k}}{\partial x_{i}}\right) \frac{\partial u_{i}}{\partial x_{k}}
$$

With the instantaneous internal energy always increasing so is the entropy, in agreement with the second law of thermodynamics. The equation can also be written

$$
\frac{\partial e}{\partial t}+u_{l} \frac{\partial e}{\partial x_{l}}=\frac{D e}{D t}=v\left(\frac{\partial u_{i}}{\partial x_{k}}+\frac{\partial u_{k}}{\partial x_{i}}\right)^{2}
$$

which shows more clearly the irreversible behavior with the square of the rate-of-strain tensor [16]. This property for the instantaneous internal energy also holds for the mean internal energy equation because the equation is derived directly from the instantaneous internal energy equation. Thus we also require

$$
\begin{aligned}
\frac{\partial \bar{e}}{\partial t}+\bar{u}_{l} \frac{\partial \bar{e}}{\partial x_{l}} & =\frac{D \bar{e}}{D t}=\bar{v}\left(\frac{\partial \bar{u}_{i}}{\partial x_{l}}+\frac{\partial \bar{u}_{l}}{\partial x_{i}}\right) \frac{\partial \bar{u}_{i}}{\partial x_{l}}+(2 \bar{v}+Q) \frac{\Delta K}{s^{2}} \\
+ & \bar{v} \frac{\partial^{2} R_{l i}}{\partial x_{l} \partial x_{i}}-2 M_{i l}\left(\frac{\partial \bar{u}_{i}}{\partial x_{l}}+\frac{\partial \bar{u}_{l}}{\partial x_{i}}\right) \geq 0
\end{aligned}
$$

The only modeled term is the source due to the decay of the turbulent kinetic energy, and this term is positive definite because $\vec{v} \geq 0$ and $K \geq 0$. Each term might not be positive 
definite, but the combination of all the terms must be positive definite. In spherical form it is not immediate the entropy change is positive definite, but using inductive reasoning we know the sum of all terms must be positive definite since the spherical form was derived from the Cartesian tensor form. The property of positive definite entropy change also holds for our spherically converging case, in which

$$
\begin{gathered}
\frac{\partial \bar{e}}{\partial t}+\bar{u}_{r} \frac{\partial \bar{e}}{\partial r}=\frac{D \bar{e}}{D t}=2 \bar{v}\left[\left(\frac{\partial \bar{u}_{r}}{\partial r}\right)^{2}+\frac{2 \bar{u}_{r}^{2}}{r^{2}}\right]+(2 \bar{v}+Q) \frac{\Delta K}{s^{2}} \\
+\frac{\bar{v}}{r^{2}} \frac{\partial}{\partial r}\left[\frac{\partial}{\partial r}\left(r^{2} R_{r r}\right)-r\left(R_{\theta \theta}+R_{\phi \phi}\right)\right]-4\left(M_{r r} \frac{\partial \bar{u}_{r}}{\partial r}+M_{\theta \theta} \frac{\bar{u}_{r}}{r}+M_{\phi \phi} \frac{\bar{u}_{r}}{r}\right) \geq 0
\end{gathered}
$$

The continuous monitoring of internal energy in our calculations thus gives an important test as to the validity of the results.

\subsection{Summary of the Properties}

The primary driver is a very important part of crenulation. The primary driver is the "seed" to creating crenulation, and without it there is no potential for this type of instability. The primary driver to crenulation is manifested through the coupling of the rate-of-strain tensor $\left(\frac{\partial \bar{u}_{i}}{\partial x_{k}}+\frac{\partial \bar{u}_{k}}{\partial x_{i}}\right)$ to the nonhomogeneity $Z_{i j}$. For the special case of spherical convergence of a spherical shell of fluid

$$
\begin{gathered}
{\left[\nabla \overline{\boldsymbol{u}}+(\nabla \overline{\boldsymbol{u}})^{T}\right]_{r r}=2 \frac{\partial \bar{u}_{r}}{\partial r}} \\
{\left[\nabla \overline{\boldsymbol{u}}+(\nabla \overline{\boldsymbol{u}})^{T}\right]_{\theta \theta}=\left[\nabla \overline{\boldsymbol{u}}+(\nabla \overline{\boldsymbol{u}})^{T}\right]_{\phi \phi}=\frac{2 \bar{u}_{r}}{r}}
\end{gathered}
$$

We have modeled the governing equations for crenulation such that when the nonhomogeneities vanish the modeled equations can still represent classical turbulence. In 
the limit as the nonhomogeneities vanish the scale, $s$, changes from the separation scale between nonhomogeneities to represent the dominant turbulence scale of the homogeneous fluid. The limit of classical turbulence transport theory is thus contained in our formulation for crenulative turbulence.

The first order approximation in combination with the cosine distribution gives a useful representation for the diagonal components of $Z_{i j}$, giving the interpretation that when $Z_{r r} \leq Z_{\theta \theta}=Z_{\phi \phi}$ then the nonhomogeneities are elongating in the radial direction and contracting in the two angular directions. The results for $Z_{i j}$ from the nonuniform distribution of the instantaneous kinematic viscosity, equation (7.12), shows the physical meaning for choices of the initial magnitude of $Z_{i j}$.

In Cartesian tensor form the dissipation function, equation (7.13) can be expressed as a sum of square terms resulting in an easily-identifiable positive-definite expression, which means the dissipation function is irreversible. In spherical form we do not have a sum of square terms, thus not being able to easily identify a positive definite dissipation function. This does not mean the dissipation function is not positive definite. In spherical form each term is not positive definite, but the sum of all terms must be positive definite since it was derived from the Cartesian tensor form, which is positive definite. 


\section{Chapter 8}

\section{Nondimensional Governing Equations, Nondimensional Initial and Boundary Conditions}

The examples that we show to describe the crenulative process are all associated with the steady-state convergence of a nonhomogeneous incompressible fluid in a spherical annulus with inner radius $R_{1}$, and outer radius, $R_{2}$. The appropriate equations are given in Chapter 6. To illustrate the behavior of our equations, we write them in nondimensional form, using the outer radius $R_{2}$ of the spherical shell, the constant parameter $B$ from the incompressibility condition discussed in Chapter 6 , the difference between the maximum and minimum values of the instantaneous kinematic viscosity, $\delta v$, and the scale of the nonhomogeneities, $s$. Nondimensionalization results in a set of equations that allow us to obtain more information from the variations of four nondimensional parameters than from five dimensional parameters. The realizability constraint on $R_{i j}, M_{i j}$, and $Z_{i j}$ is a guidance for the development of the nondimensional boundary conditions. Our calculations develop the steady-state solution through time evolution from prescribed initial conditions, but it is only the final steady state that we illustrate in this thesis. Thus we start each calculation with the convenient configuration of uniform distribution of $\hat{Z}_{i j}$ and with inflow of $\hat{Z}_{i j}$ through $R_{2}$, and follow the dynamics until there are no residual stochastic variations in time. 


\subsection{Nondimensional Governing Equations}

Nondimensionalizing the governing equations results in three nondimensional parameters. To obtain these nondimensional parameters the radius, time, $R_{i j}, M_{i j}$, and $Z_{i j}$ are all nondimensionalized in the following manner

$$
\begin{gathered}
r=\hat{r}_{2} \quad t=\hat{t} \frac{R_{2}^{3}}{|B|} \\
R_{i j}=\hat{R}_{i j}\left(\frac{B}{R_{2}^{2}}\right)^{2} \quad M_{i j}=\hat{M}_{i j}\left(\frac{\delta V}{s}\right)^{2} \quad Z_{i j}=\hat{Z}_{i j}\left(\frac{\delta v}{s}\right)^{2} \quad \bar{e}=\hat{\bar{e}}\left(\frac{B}{R_{2}^{2}}\right)^{2}
\end{gathered}
$$

The nondimensional radius is $\hat{r}, \hat{t}$ is the nondimensional time, $\hat{R}_{i j}$ is the nondimensional $R_{i j}, \hat{M}_{i j}$ is the nondimensional $M_{i j}, \hat{Z}_{i j}$ is the nondimensional $Z_{i j}$, and $\hat{\bar{e}}$ is the nondimensional $\bar{e}$. The radius is nondimensionalized with $R_{2}$. The time is nondimensionalized with a distance/velocity, where $R_{2}$ is the distance and $|B| / R_{2}^{2}$ is the magnitude of mean velocity at the outer radius of the shell. Nondimensionalizing with $|B|$ ensures the time evolution is positive for both convergence and divergence of the spherical shell of fluid. The Reynolds stress and the internal energy are both nondimensionalized by the mean velocity at the outer radius of the shell $\left(|B| / R_{2}^{2}\right)^{2}$. The quantities $M_{i j}$ and $Z_{i j}$ are both nondimensionalized by $(\delta v / s)^{2}$, which is suggested by the result of equations (7.7) with the cosine distribution analysis done in section 7.4. The three nondimensional parameters that arise in the governing equations from the nondimensionalization are

$$
\operatorname{Re}=\frac{|B|}{\bar{v} R_{2}} \quad \beta=\frac{s}{R_{2}} \quad \gamma=\frac{\delta V}{\bar{v}}
$$

The nondimensional parameter $\frac{|B|}{\bar{v} R_{2}}$ is a Reynolds number, because a reference velocity can be defined as $\bar{u}_{\text {reference }}=\frac{|B|}{R_{2}^{2}}$, so that 


$$
\operatorname{Re}=\frac{\bar{u}_{\text {reference }} R_{2}}{\bar{v}}=\frac{\left(\frac{|B|}{R_{2}^{2}}\right) R_{2}}{\bar{v}}=\frac{|B|}{\bar{v} R_{2}}
$$

The nondimensional parameter $\beta$ is a measure of the scale of the nonhomogeneity relative to the size of the spherical shell. The nondimensional parameter $\gamma$ is a measure of the level of nonhomogeneity in the spherical shell. We also define $\eta=\frac{B}{|B|}$, which is positive if the flow is diverging and negative if the flow is converging. The nondimensional form of the individual components of the Reynolds stress equation are

$$
\begin{aligned}
& \frac{\partial \hat{R}_{r r}}{\partial \hat{t}}-4 \eta \frac{\hat{R}_{r r}}{\hat{r}^{3}}+\eta \frac{1}{\hat{r}^{2}} \frac{\partial \hat{R}_{r r}}{\partial \hat{r}}=\frac{1}{\hat{r}^{2}} \frac{\partial}{\partial \hat{r}}\left[\hat{r}^{2} 2 C_{D} \beta \sqrt{\hat{K}} \frac{\partial \hat{R}_{r r}}{\partial \hat{r}}\right] \\
& -4 C_{D} \beta \sqrt{\hat{K}} \frac{\left(2 \hat{R}_{r r}-\hat{R}_{\theta \theta}-\hat{R}_{\phi \phi}\right)}{\hat{r}^{2}}+C_{B} \eta\left[-4 \frac{\hat{R}_{r r}}{\hat{r}^{3}}-\frac{2}{3}\left(-2 \frac{\hat{R}_{r r}}{\hat{r}^{3}}+\frac{\hat{R}_{\theta \theta}}{\hat{r}^{3}}+\frac{\hat{R}_{\phi \phi}}{\hat{r}^{3}}\right)\right] \\
& +\left(\frac{C_{R} \sqrt{\hat{K}}}{\beta}+\frac{C_{H R} \sqrt{6}}{\hat{r}^{3}}\right)\left(\frac{2}{3} \hat{K}-\hat{R}_{r r}\right)-8 \eta\left(\frac{\gamma}{\operatorname{Re} \beta}\right)^{2} \frac{\hat{M}_{r r}}{\hat{r}^{3}} \\
& +\frac{1}{\operatorname{Re}}\left[\frac{1}{\hat{r}^{2}} \frac{\partial}{\partial \hat{r}}\left(\hat{r}^{2} \frac{\partial \hat{R}_{r r}}{\partial \hat{r}}\right)-2 \frac{\left(2 \hat{R}_{r r}-\hat{R}_{\theta \theta}-\hat{R}_{\phi \phi}\right)}{\hat{r}^{2}}\right]-\frac{1}{\operatorname{Re} \beta^{2}}\left(2+w_{1}\right) \Delta \hat{R}_{r r} \\
& \frac{\partial \hat{R}_{\theta \theta}}{\partial \hat{t}}+2 \eta \frac{\hat{R}_{\theta \theta}}{\hat{r}^{3}}+\eta \frac{1}{\hat{r}^{2}} \frac{\partial \hat{R}_{\theta \theta}}{\partial \hat{r}}=\frac{1}{\hat{r}^{2}} \frac{\partial}{\partial \hat{r}}\left[\hat{r}^{2} 2 C_{D} \beta \sqrt{\hat{K}} \frac{\partial \hat{R}_{\theta \theta}}{\partial \hat{r}}\right] \\
& +4 C_{D} \beta \sqrt{\hat{K}} \frac{\left(\hat{R}_{r r}-\hat{R}_{\theta \theta}\right)}{\hat{r}^{2}}+C_{B} \eta\left[2 \frac{\hat{R}_{\theta \theta}}{\hat{r}^{3}}-\frac{2}{3}\left(-2 \frac{\hat{R}_{r r}}{\hat{r}^{3}}+\frac{\hat{R}_{\theta \theta}}{\hat{r}^{3}}+\frac{\hat{R}_{\phi \phi}}{\hat{r}^{3}}\right)\right] \\
& +\left(\frac{C_{R} \sqrt{\hat{K}}}{\beta}+\frac{C_{H R} \sqrt{6}}{\hat{r}^{3}}\right)\left(\frac{2}{3} \hat{K}-\hat{R}_{\theta \theta}\right)+4 \eta\left(\frac{\gamma}{\operatorname{Re} \beta}\right)^{2} \frac{\hat{M}_{\theta \theta}}{\hat{r}^{3}} \\
& +\frac{1}{\operatorname{Re}}\left[\frac{1}{\hat{r}^{2}} \frac{\partial}{\partial \hat{r}}\left(\hat{r}^{2} \frac{\partial \hat{R}_{r r}}{\partial \hat{r}}\right)+2 \frac{\left(\hat{R}_{r r}-\hat{R}_{\theta \theta}\right)}{\hat{r}^{2}}\right]-\frac{1}{\operatorname{Re} \beta^{2}}\left(2+w_{1}\right) \Delta \hat{R}_{\theta \theta}
\end{aligned}
$$




$$
\begin{gathered}
\frac{\partial \hat{R}_{\phi \phi}}{\partial \hat{t}}+2 \eta \frac{\hat{R}_{\phi \phi}}{\hat{r}^{3}}+\eta \frac{1}{\hat{r}^{2}} \frac{\partial \hat{R}_{\phi \phi}}{\partial \hat{r}}=\frac{1}{\hat{r}^{2}} \frac{\partial}{\partial \hat{r}}\left[\hat{r}^{2} 2 C_{D} \beta \sqrt{\hat{K}} \frac{\partial \hat{R}_{\phi \phi}}{\partial \hat{r}}\right] \\
+4 C_{D} \beta \sqrt{\hat{K}} \frac{\left(\hat{R}_{r r}-\hat{R}_{\phi \phi}\right)}{\hat{r}^{2}}+C_{B} \eta\left[2 \frac{\hat{R}_{\phi \phi}}{\hat{r}^{3}}-\frac{2}{3}\left(-2 \frac{\hat{R}_{r r}}{\hat{r}^{3}}+\frac{\hat{R}_{\theta \theta}}{\hat{r}^{3}}+\frac{\hat{R}_{\phi \phi}}{\hat{r}^{3}}\right)\right] \\
+\left(\frac{C_{R} \sqrt{\hat{K}}}{\beta}+\frac{C_{H R} \sqrt{6}}{\hat{r}^{3}}\right)\left(\frac{2}{3} \hat{K}-\hat{R}_{\phi \phi}\right)+4 \eta\left(\frac{\gamma}{\operatorname{Re} \beta}\right)^{2} \frac{\hat{M}_{\phi \phi}}{\hat{r}^{3}} \\
+\frac{1}{\operatorname{Re}}\left[\frac{1}{\hat{r}^{2}} \frac{\partial}{\partial \hat{r}}\left(\hat{r}^{2} \frac{\partial \hat{R}_{r r}}{\partial \hat{r}}\right)+2 \frac{\left(\hat{R}_{r r}-\hat{R}_{\phi \phi}\right)}{\hat{r}^{2}}\right]-\frac{1}{\operatorname{Re} \beta^{2}}\left(2+w_{1}\right) \Delta \hat{R}_{\phi \phi}
\end{gathered}
$$

The nondimensional form of the individual components of the $M_{i j}$ equation are

$$
\begin{aligned}
& \frac{\partial \hat{M}_{r r}}{\partial \hat{t}}-4 \eta \frac{\hat{M}_{r r}}{\hat{r}^{3}}-\frac{2}{3} \eta\left(-2 \frac{\hat{M}_{r r}}{\hat{r}^{3}}+\frac{\hat{M}_{\theta \theta}}{\hat{r}^{3}}+\frac{\hat{M}_{\phi \phi}}{\hat{r}^{3}}\right)+\eta \frac{1}{\hat{r}^{2}} \frac{\partial \hat{M}_{r r}}{\partial \hat{r}}-\frac{1}{\hat{r}^{2}} \frac{\partial}{\partial \hat{r}}\left[\hat{r}^{2} 2 C_{H} \beta \sqrt{\hat{K}} \frac{\partial \hat{M}_{r r}}{\partial \hat{r}}\right] \\
& +4 C_{H} \beta \sqrt{\hat{K}} \frac{\left(2 \hat{M}_{r r}-\hat{M}_{\theta \theta}-\hat{M}_{\phi \phi}\right)}{\hat{r}^{2}}=-\left[\frac{\left(2+w_{2}\right) \Delta}{\operatorname{Re} \beta^{2}}+\frac{C_{M} \sqrt{\hat{K}}}{\beta}+\frac{C_{H R} \sqrt{6}}{\hat{r}^{3}}\right] \hat{M}_{r r} \\
& -4 \eta \frac{\hat{Z}_{r r}}{\hat{r}^{3}}-\frac{2}{3} \eta\left(-2 \frac{\hat{Z}_{r r}}{\hat{r}^{3}}+\frac{\hat{Z}_{\theta \theta}}{\hat{r}^{3}}+\frac{\hat{Z}_{\phi \phi}}{\hat{r}^{3}}\right) \\
& +\frac{1}{\operatorname{Re}} \frac{1}{\hat{r}^{2}} \frac{\partial}{\partial \hat{r}}\left[\hat{r}^{2} \frac{\partial \hat{M}_{r r}}{\partial \hat{r}}\right]-\frac{2}{\operatorname{Re}} \frac{\left(2 \hat{M}_{r r}-\hat{M}_{\theta \theta}-\hat{M}_{\phi \phi}\right)}{\hat{r}^{2}} \\
& \frac{\partial \hat{M}_{\theta \theta}}{\partial \hat{t}}+2 \eta \frac{\hat{M}_{\theta \theta}}{\hat{r}^{3}}-\frac{2}{3} \eta\left(-2 \frac{\hat{M}_{r r}}{\hat{r}^{3}}+\frac{\hat{M}_{\theta \theta}}{\hat{r}^{3}}+\frac{\hat{M}_{\phi \phi}}{\hat{r}^{3}}\right)+\eta \frac{1}{\hat{r}^{2}} \frac{\partial \hat{M}_{\theta \theta}}{\partial \hat{r}}-\frac{1}{\hat{r}^{2}} \frac{\partial}{\partial \hat{r}}\left[\hat{r}^{2} 2 C_{H} \beta \sqrt{\hat{K}} \frac{\partial \hat{M}_{\theta \theta}}{\partial \hat{r}}\right] \\
& -4 C_{H} \beta \sqrt{\hat{K}} \frac{\left(\hat{M}_{r r}-\hat{M}_{\theta \theta}\right)}{\hat{r}^{2}}=-\left[\frac{\left(2+w_{2}\right) \Delta}{\operatorname{Re} \beta^{2}}+\frac{C_{M} \sqrt{\hat{K}}}{\beta}+\frac{C_{H R} \sqrt{6}}{\hat{r}^{3}}\right] \hat{M}_{\theta \theta} \\
& +2 \eta \frac{\hat{Z}_{\theta \theta}}{\hat{r}^{3}}-\frac{2}{3} \eta\left(-2 \frac{\hat{Z}_{r r}}{\hat{r}^{3}}+\frac{\hat{Z}_{\theta \theta}}{\hat{r}^{3}}+\frac{\hat{Z}_{\phi \phi}}{\hat{r}^{3}}\right) \\
& +\frac{1}{\operatorname{Re}} \frac{1}{\hat{r}^{2}} \frac{\partial}{\partial \hat{r}}\left[\hat{r}^{2} \frac{\partial \hat{M}_{\theta \theta}}{\partial \hat{r}}\right]+\frac{2}{\operatorname{Re}} \frac{\left(\hat{M}_{r r}-\hat{M}_{\theta \theta}\right)}{\hat{r}^{2}}
\end{aligned}
$$




$$
\begin{gathered}
\frac{\partial \hat{M}_{\phi \phi}}{\partial \hat{t}}+2 \eta \frac{\hat{M}_{\phi \phi}}{\hat{r}^{3}}-\frac{2}{3} \eta\left(-2 \frac{\hat{M}_{r r}}{\hat{r}^{3}}+\frac{\hat{M}_{\theta \theta}}{\hat{r}^{3}}+\frac{\left.\hat{M}_{\phi \phi}\right)}{\hat{r}^{3}}\right)+\eta \frac{1}{\hat{r}^{2}} \frac{\partial \hat{M}_{\phi \phi}}{\partial \hat{r}}-\frac{1}{\hat{r}^{2}} \frac{\partial}{\partial \hat{r}}\left[\hat{r}^{2} 2 C_{H} \beta \sqrt{\hat{K}} \frac{\partial \hat{M}_{\phi \phi}}{\partial \hat{r}}\right] \\
-4 C_{H} \beta \sqrt{\hat{K}} \frac{\left(\hat{M}_{r r}-\hat{M}_{\phi \phi}\right)}{\hat{r}^{2}}=-\left[\frac{\left(2+w_{2}\right) \Delta}{\operatorname{Re} \beta^{2}}+\frac{C_{M} \sqrt{\hat{K}}}{\beta}+\frac{C_{H R} \sqrt{6}}{\hat{r}^{3}}\right] \hat{M}_{\phi \phi} \\
+2 \eta \frac{\hat{Z}_{\phi \phi}}{\hat{r}^{3}}-\frac{2}{3} \eta\left(-2 \frac{\hat{Z}_{r r}}{\hat{r}^{3}}+\frac{\hat{Z}_{\theta \theta}}{\hat{r}^{3}}+\frac{\hat{Z}_{\phi \phi}}{\hat{r}^{3}}\right) \\
+\frac{1}{\operatorname{Re}} \frac{1}{\hat{r}^{2}} \frac{\partial}{\partial \hat{r}}\left[\hat{r}^{2} \frac{\partial \hat{M}_{\phi \phi}}{\partial \hat{r}}\right]+\frac{2}{\operatorname{Re}} \frac{\left(\hat{M}_{r r}-\hat{M}_{\phi \phi}\right)}{\hat{r}^{2}}
\end{gathered}
$$

The nondimensional form of the individual components of the $Z_{i j}$ equation are

$$
\begin{aligned}
& \frac{\partial \hat{Z}_{r r}}{\partial \hat{t}}-4 \eta \frac{\hat{Z}_{r r}}{\hat{r}^{3}}-\frac{2}{3} \eta\left(-2 \frac{\hat{Z}_{r r}}{\hat{r}^{3}}+\frac{\hat{Z}_{\theta \theta}}{\hat{r}^{3}}+\frac{\hat{Z}_{\phi \phi}}{\hat{r}^{3}}\right)+\eta \frac{1}{\hat{r}^{2}} \frac{\partial \hat{Z}_{r r}}{\partial \hat{r}} \\
& =\left[\frac{C_{Z} \sqrt{\hat{K}}}{\beta}+\frac{C_{H R} \sqrt{6}}{\hat{r}^{3}}\right]\left[\frac{1}{3}\left(\hat{Z}_{r r}+\hat{Z}_{\theta \theta}+\hat{Z}_{\phi \phi}\right)-\hat{Z}_{r r}\right]+\frac{1}{\hat{r}^{2}} \frac{\partial}{\partial \hat{r}}\left[\hat{r}^{2} 2 C_{X} \beta \sqrt{\hat{K}} \frac{\partial \hat{Z}_{r r}}{\partial \hat{r}}\right] \\
& -4 C_{X} \beta \sqrt{\hat{K}} \frac{\left(2 \hat{Z}_{r r}-\hat{Z}_{\theta \theta}-\hat{Z}_{\phi \phi}\right)}{\hat{r}^{2}}-\frac{1}{3} \frac{1}{\hat{r}^{2}} \frac{\partial}{\partial \hat{r}}\left[\hat{r}^{2} 2 C_{X} \beta \sqrt{\hat{K}} \frac{\partial\left(\hat{Z}_{r r}+\hat{Z}_{\theta \theta}+\hat{Z}_{\phi \phi}\right)}{\partial \hat{r}}\right] \\
& \frac{\partial \hat{Z}_{\theta \theta}}{\partial \hat{t}}+2 \eta \frac{\hat{Z}_{\theta \theta}}{\hat{r}^{3}}-\frac{2}{3} \eta\left(-2 \frac{\hat{Z}_{r r}}{\hat{r}^{3}}+\frac{\hat{Z}_{\theta \theta}}{\hat{r}^{3}}+\frac{\hat{Z}_{\phi \phi}}{\hat{r}^{3}}\right)+\eta \frac{1}{\hat{r}^{2}} \frac{\partial \hat{Z}_{\theta \theta}}{\partial r} \\
& =\left[\frac{C_{Z} \sqrt{\hat{K}}}{\beta}+\frac{C_{H R} \sqrt{6}}{\hat{r}^{3}}\right]\left[\frac{1}{3}\left(\hat{Z}_{r r}+\hat{Z}_{\theta \theta}+\hat{Z}_{\phi \phi}\right)-\hat{Z}_{\theta \theta}\right]+\frac{1}{\hat{r}^{2}} \frac{\partial}{\partial \hat{r}}\left[\hat{r}^{2} 2 C_{X} \beta \sqrt{\hat{K}} \frac{\partial \hat{Z}_{\theta \theta}}{\partial \hat{r}}\right] \\
& +4 C_{X} \beta \sqrt{\hat{K}} \frac{\left(\hat{Z}_{r r}-\hat{Z}_{\theta \theta}\right)}{\hat{r}^{2}}-\frac{1}{3} \frac{1}{\hat{r}^{2}} \frac{\partial}{\partial \hat{r}}\left[\hat{r}^{2} 2 C_{X} \beta \sqrt{\hat{K}} \frac{\partial\left(\hat{Z}_{r r}+\hat{Z}_{\theta \theta}+\hat{Z}_{\phi \phi}\right)}{\partial \hat{r}}\right]
\end{aligned}
$$




$$
\begin{gathered}
\frac{\partial \hat{Z}_{\phi \phi}}{\partial \hat{t}}+2 \eta \frac{\hat{Z}_{\phi \phi}}{\hat{r}^{3}}-\frac{2}{3} \eta\left(-2 \frac{\hat{Z}_{r r}}{\hat{r}^{3}}+\frac{\hat{Z}_{\theta \theta}}{\hat{r}^{3}}+\frac{\hat{Z}_{\phi \phi}}{\hat{r}^{3}}\right)+\eta \frac{1}{\hat{r}^{2}} \frac{\partial \hat{Z}_{\phi \phi}}{\partial r} \\
=\left[\frac{C_{Z} \sqrt{\hat{K}}}{\beta}+\frac{C_{H R} \sqrt{6}}{\hat{r}^{3}}\right]\left[\frac{1}{3}\left(\hat{Z}_{r r}+\hat{Z}_{\theta \theta}+\hat{Z}_{\phi \phi}\right)-\hat{Z}_{\phi \phi}\right]+\frac{1}{\hat{r}^{2}} \frac{\partial}{\partial \hat{r}}\left[\hat{r}^{2} 2 C_{X} \beta \sqrt{\hat{K}} \frac{\partial \hat{Z}_{\phi \phi}}{\partial \hat{r}}\right] \\
+4 C_{X} \beta \sqrt{\hat{K}} \frac{\left(\hat{Z}_{r r}-\hat{Z}_{\phi \phi}\right)}{\hat{r}^{2}}-\frac{1}{3} \frac{1}{\hat{r}^{2}} \frac{\partial}{\partial \hat{r}}\left[\hat{r}^{2} 2 C_{X} \beta \sqrt{\hat{K}} \frac{\partial\left(\hat{Z}_{r r}+\hat{Z}_{\theta \theta}+\hat{Z}_{\phi \phi}\right)}{\partial \hat{r}}\right]
\end{gathered}
$$

The nondimensional form of the internal energy equation is

$$
\begin{array}{r}
\frac{\partial \hat{\bar{e}}}{\partial \hat{t}}+\eta \frac{1}{\hat{r}^{2}} \frac{\partial \hat{\bar{e}}}{\partial \hat{r}}=\frac{12}{\operatorname{Re} \hat{r}^{6}}+\frac{\left(2+w_{1}\right) \Delta \hat{K}}{\operatorname{Re} \beta^{2}}+\frac{1}{\operatorname{Re} \hat{r}^{2}} \frac{\partial}{\partial \hat{r}}\left[\frac{\partial}{\partial \hat{r}}\left(\hat{r}^{2} \hat{R}_{r r}\right)-\hat{r}\left(\hat{R}_{\theta \theta}+\hat{R}_{\phi \phi}\right)\right] \\
-4 \eta\left(\frac{\gamma}{\operatorname{Re} \beta}\right)^{2}\left(-2 \frac{\hat{M}_{r r}}{\hat{r}^{3}}+\frac{\hat{M}_{\theta \theta}}{\hat{r}^{3}}+\frac{\hat{M}_{\phi \phi}}{\hat{r}^{3}}\right)
\end{array}
$$

The numerical scheme used to solve the set of governing equations (8.1), (8.2), (8.3), and (8.4) is an explicit time marching finite difference technique described in Appendix A, section 1 .

\subsection{Nondimensional Initial and Boundary Conditions}

The initial conditions appear not to effect the steady-state solution (unless the evolution diverges), but we have chosen these conditions such that the nondimensional realizability constraint

$$
\hat{R}_{(i i)} \hat{Z}_{(j j)}\left(\frac{\operatorname{Re} \beta}{\gamma}\right)^{2}-\hat{M}_{(i j)}^{2} \geq 0
$$

is not violated, where the parenthesis indicate no summation. The initial condition for

$\hat{M}_{(i j)}$ is zero; this condition satisfies the nondimensional realizability constraint for all 
positive choices of $\hat{R}_{(i i)}$ and $\hat{Z}_{(j j)}$. The initial condition for the internal energy $\hat{\bar{e}}$ is zero; this condition means the fluid is in a "cold" state.

The boundary conditions at the outer radius of the spherical system must be specified, since it is these values that will be fluxed in through the boundary. The outer boundary condition for $\hat{M}_{(i j)}$ is the same as its initial condition, zero. The outer boundary condition for $\hat{R}_{(i i)}$ is chosen such that the turbulence energy is ten percent of the mean kinetic energy at the outer radius., i.e. at the outer boundary. Also, $\hat{R}_{i j}$ does not have to be isotropic. The outer boundary condition for $\hat{Z}_{(j j)}$ is a choice of the level of nonhomogeneity wanted in the system. From the cosine distribution in Chapter 7, equation (7.9), we would choose

$$
\hat{Z}_{(j j)}=\frac{\pi^{2}}{8}
$$

but this relationship introduces only a low level crenulative consequences. Instead we choose to use equation (7.12), from the nonuniform distribution in Chapter 7, to obtain $\hat{Z}_{(j j)}$ at the outer boundary

$$
\hat{Z}_{(j j)}=\frac{4 \pi \bar{R}^{2}}{s a}
$$

This relationship for $\hat{Z}_{(j j)}$ allows us to choose any level of nonhomogeneity for the boundary condition. In all cases we specify $\hat{Z}_{i j}$ to be isotropic at the inlet boundary. The outer boundary condition for the internal energy $\hat{\bar{e}}$ is the same as its initial condition, zero. The advective boundary condition at the inner radius of the spherical system does not have to be specified; the diffusive boundary conditions are. The boundary conditions are extrapolative, with implementation that is described more fully in Appendix A, section 2. 
These boundary conditions introduce several additional dimensionless parameters to complete the specification of each calculation. Besides the values of $\operatorname{Re}, \beta$, and $\gamma$ the important boundary-condition parameter that we vary is $\hat{Z}_{(j j)}$. 


\section{Chapter 9}

\section{Results and Conclusions}

In the nondimensional parameter space of $\operatorname{Re}, \beta, \gamma$, and $\hat{Z}_{(j i)}$ many variations could be analyzed to illustrate crenulation; here we have chosen a small set to display some of the trends. All of these results were analyzed at steady state and with a coarse resolution in the finite-difference calculations. First, we show the analytic solution for the homogeneous case, then we show a coarse resolution calculation of the homogeneous case and compare it to a coarse resolution calculation of a case (case A) in which there is a high level of nonhomogeneity. Second, we choose a base case, which has a moderate level of nonhomogeneity, and compare the internal energy to the internal energy of the homogeneous case. We also look at the different energy modes, $\hat{M}_{i j}$ component distributions, and $\hat{Z}_{i j}$ component distributions for the base case. Third, we make two Reynolds number variations from the base case and look at the internal energy and turbulence energy. Fourth, we make one $\beta$ variation from the base case and look at the internal energy and turbulence energy. Fifth, we make one $\gamma$ variation from the base case and look at the internal energy and turbulence energy. Sixth, we make two $\hat{Z}_{(j j)}$ variations from the base case and look at the internal energy and turbulence energy. Finally, we return to the base case and enhance a modeling constant in the return to isotropy in the $\hat{Z}_{i j}$ equation and only look at the $\hat{Z}_{(j j)}$ distribution.

The coarse resolution used is sufficient because the differences seen in the fineresolution calculations were also seen in the coarse-resolution calculations. It is not the focus of this thesis to do an in-depth analysis of the numerical algorithm, but to analyze the trends associated with crenulative turbulence. 


\subsection{Homogeneous Case}

An analytic solution for the internal energy can be obtained when the crenulative fluctuations vanish, i.e. the homogeneous case. Consider the behavior of internal energy given by equation (2.12), with its instantaneous quantities and in spherical form

$$
\frac{\partial e}{\partial t}+u_{r} \frac{\partial e}{\partial r}=2 v\left[\left(\frac{\partial u_{r}}{\partial r}\right)^{2}+\frac{2 u_{r}^{2}}{r^{2}}\right]
$$

In steady state the time derivative vanishes. Nondimensionalizing the rest of equation (9.1) results in the following

$$
\frac{\partial \hat{e}}{\partial \hat{r}}=\frac{12}{\eta \operatorname{Re}} \frac{1}{\hat{r}^{4}}
$$

The solution to equation (9.2) is

$$
\hat{e}\left(\hat{R}_{2}\right)-\hat{e}(\hat{r})=\frac{-4}{\eta \operatorname{Re}}\left(\frac{1}{\hat{R}_{2}^{3}}-\frac{1}{\hat{r}^{3}}\right)
$$

The boundary condition at the outer radius is zero for the internal energy and the nondimensional outer radius $\hat{R}_{2}$ is equal to one, so that the solution becomes

$$
\hat{e}(\hat{r})=\frac{4}{\eta \operatorname{Re}}\left(1-\frac{1}{\hat{r}^{3}}\right)
$$

This homogeneous case can be compared to a case in which a level of nonhomogeneity is present. In this case, case A, we have chosen the nondimensional parameters as 


$$
\text { Case A: } \operatorname{Re}=100 \quad \beta=0.05 \quad \gamma=0.9 \quad \hat{Z}_{(j j)}=200
$$

where the parenthesis indicate no summation. Case A represents a high level of nonhomogeneity in the spherical shell of fluid. In this comparison we can see in Fig. 9.1 that there is a significant drop in internal energy, in comparison with the homogeneous case.

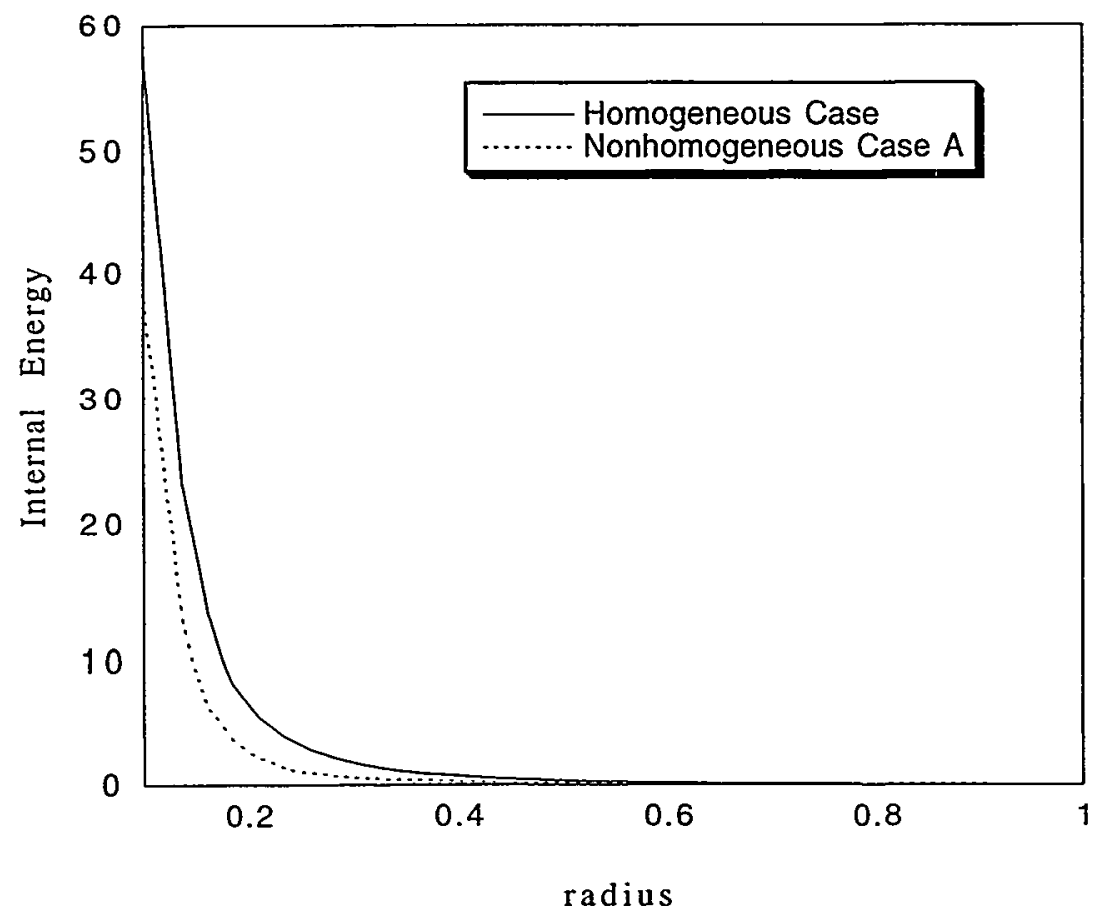

Figure 9.1: Comparison of Homogeneous Case to Nonhomogeneous Case A

In order to illustrate this contrast, the homogeneous results in Fig. 9.1 are numerical results with the same resolution as for the nonhomogeneous case. The comparison between numerical and analytical solutions for the homogeneous case are shown and discussed in Appendix A, section 3. In particular, we have shown that calculations for both cases with coarse and fine resolution give essentially the same difference as shown in Fig. 9.1. The drop in internal energy is a direct result of the nonhomogeneous variations of kinematic 
viscosity. The high regions of kinematic viscosity slip with little internal strain through the low regions of kinematic viscosity, with the net effect of less dissipation to heat. In the homogeneous case the strain is distributed throughout the viscous fluid, giving more resistance to converging, thus more dissipation to heat. Note that a large value of incoming $\hat{Z}_{(j)}$ is required to exhibit this contrast in dissipation. As shown in equation (8.6), a large magnitude of $\hat{Z}_{(j)}$ indicates that the regions of high viscosity fill most of the volume, with narrow transitional regions to the surrounding low viscosity. The overall mean viscosity, $\bar{v}$, is not much different from the maximum value of $v$. If the fluid contorts with a homogeneous distribution of that $\bar{v}$, the dissipation to heat is large; but the presence of very narrow slip channels of low viscosity allows the crenulative strain to take place mainly in those channel regions, so that the net dissipation is significantly decreased. This result demonstrates one of the principal conclusions of this study.

\subsection{Base Case}

The nondimensional parameters, $\operatorname{Re}, \beta, \gamma$, and the level of nonhomogeneity in the system, $\hat{Z}_{(j j)}$, are all parameters that can be varied. As a base case we have chosen the nondimensional parameters and the level of nonhomogeneity to have the following magnitudes

$$
\text { Base Case: } \operatorname{Re}=100 \quad \beta=0.05 \quad \gamma=0.5 \quad \hat{Z}_{(j j)}=100
$$

Because $\hat{Z}_{(j j)}$ and $\beta$ are less for the base case than for case $\mathrm{A}$, there is a lower degree of nonhomogeneity. This means the base case is closer than case A to the homogeneous case. This can be seen in Fig. 9.2 where the internal energy for the nonhomogeneous base case is only slightly lower than that of the homogeneous case. 


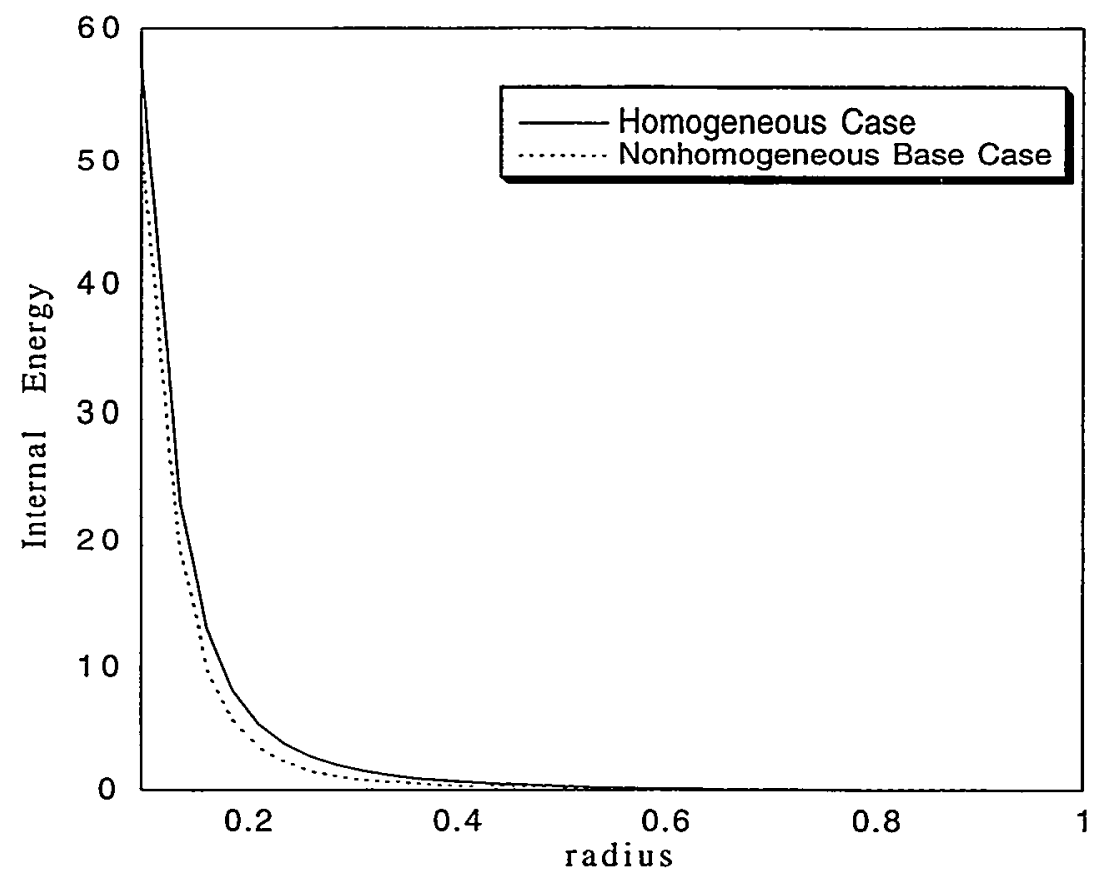

Figure 9.2: Comparison of Homogeneous Case to Nonhomogeneous Base Case

In the base case the mean kinetic energy is very large at the inner radius of the spherical shell of fluid in comparison to the internal energy and the turbulence energy, as shown in Fig. 9.3. 


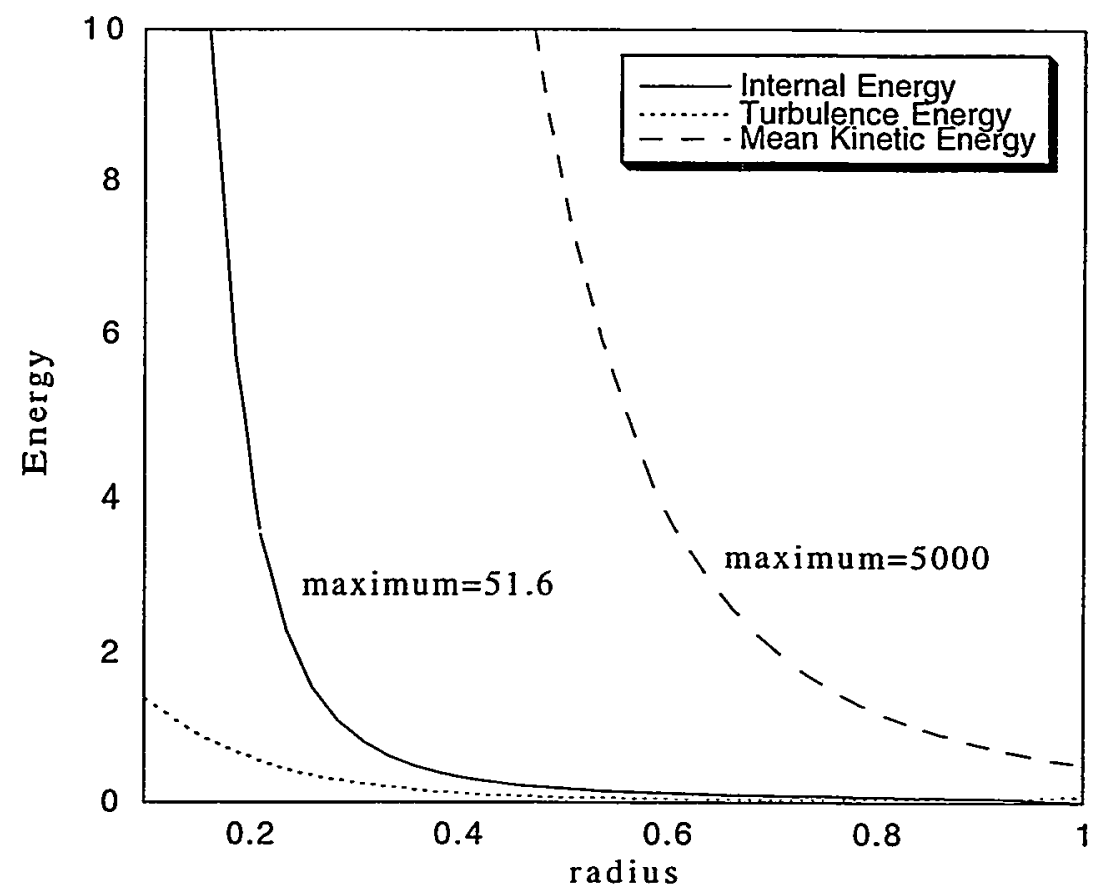

Figure 9.3: Energies for the Nonhomogeneous Base Case

The $r r$-component of $\hat{M}_{i j}$ is positive and increases sharply near the inner radius, while the $\theta \theta$-component of $\hat{M}_{i j}$ is negative and decreases near the inner radius, as can be seen in Fig. 9.4. Both behaviors are linked because of the vanishing contraction of $\hat{M}_{i j}$

$$
\hat{M}_{l l}=\hat{M}_{r r}+\hat{M}_{\theta \theta}+\hat{M}_{\phi \phi}=0
$$

Because the system is spherically symmetric, the $\theta \theta$-component is the same as the $\phi \phi$ component, so that

$$
\hat{M}_{r r}=-2 \hat{M}_{\theta \theta}
$$


The $\hat{M}_{i j}$ components are a first order response to the crenulation and act as a bridge between $\hat{Z}_{i j}$ and $\hat{R}_{i j}$.

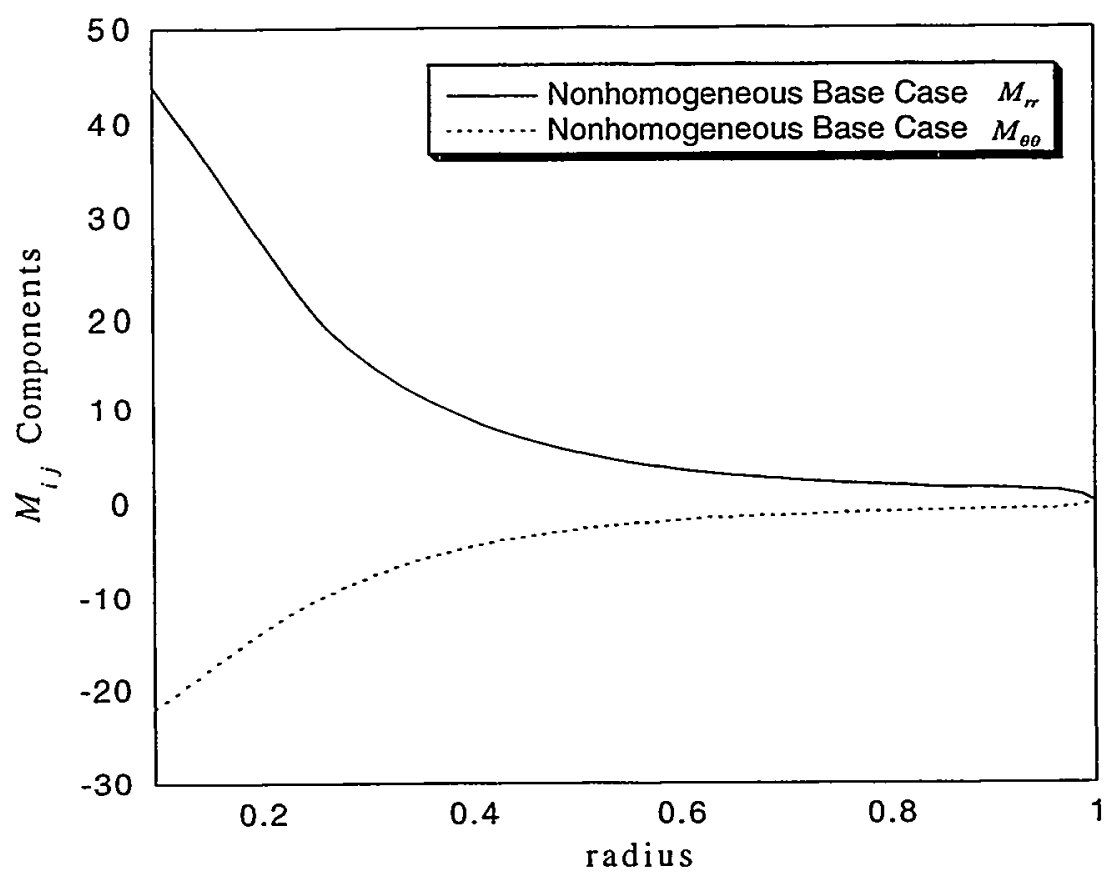

Figure 9.4: $\hat{M}_{i j}$ Components for the Base Case

The results of the calculation for $\hat{Z}_{i j}$ for the base case show, in Fig. 9.5, the trends discussed in Chapter 7 sections 3 and 4 , in which $\hat{Z}_{r r} \leq \hat{Z}_{\theta \theta}=\hat{Z}_{\phi \phi}$ implies elongation of the nonhomogeneities in the radial direction. 


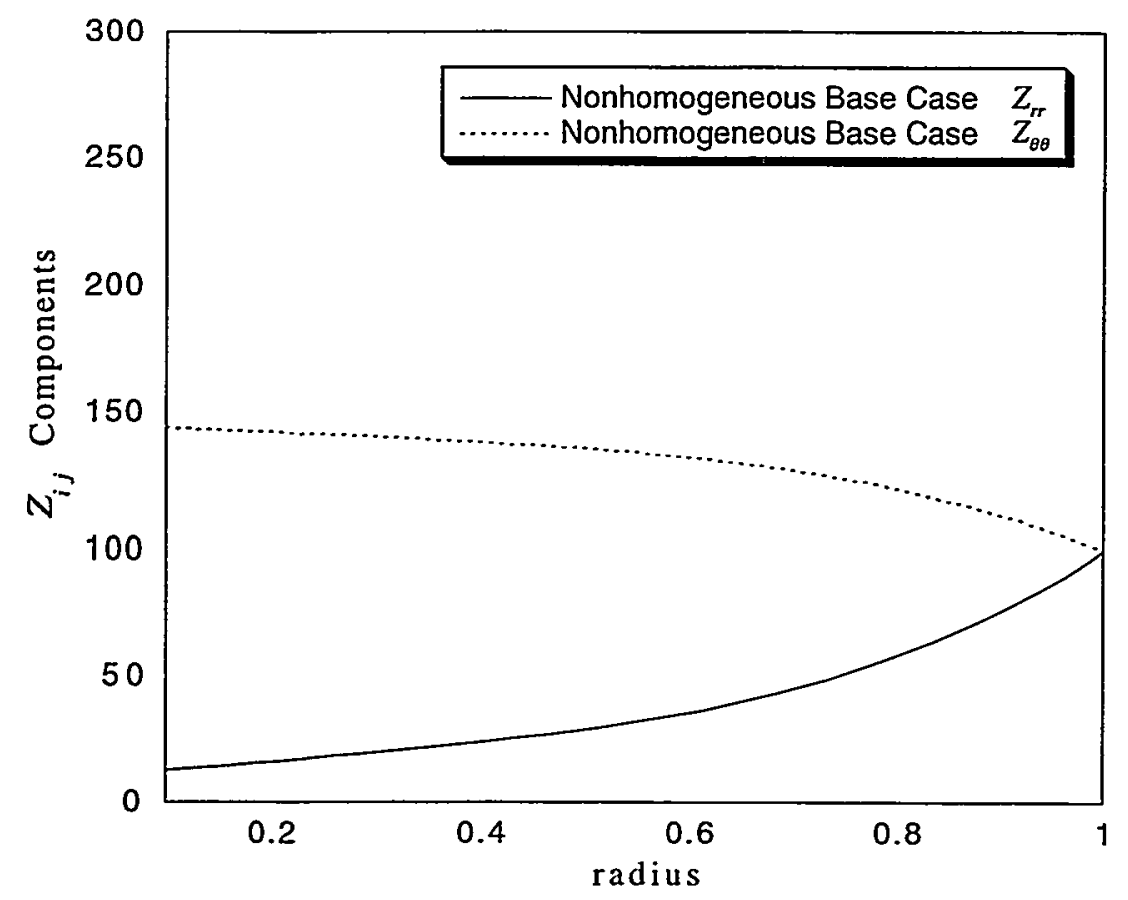

Figure 9.5: $\hat{Z}_{i j}$ Components for the Base Case

The component structure of $\hat{Z}_{i j}$ is mainly a result of kinematic response. There is very little dependence on how fast the spherical shell is converging. Almost the same distribution as in Fig. 9.5 occurs for both higher and lower Reynolds numbers. We also see the same distribution for both higher and lower values of $\beta$. We also see the same distribution for a lower value of $\gamma$, but for higher $\gamma$ we see a slight variation from the base case near the inner radius. 


\subsection{Variations of Reynolds Number}

Varying only the Reynolds number from the base case has effects on the internal energy and the turbulence energy. Because the variations in $\hat{Z}_{i j}$ are mainly due to kinematic effects the results for $\hat{Z}_{i j}$ in case $B$ and case $C$ are the same as for the base case. We have chosen Reynolds numbers both smaller and larger than in the base case.

$$
\begin{aligned}
& \text { Case B: } \operatorname{Re}=50 \quad \beta=0.05 \quad \gamma=0.5 \quad \hat{Z}_{(j)}=100 \\
& \text { Case C: } \operatorname{Re}=200 \quad \beta=0.05 \quad \gamma=0.5 \quad \hat{Z}_{(j j)}=100
\end{aligned}
$$

In the homogeneous version of case B the dimensionless internal energy is larger than that of the homogeneous case with $\mathrm{Re}=100$, as seen by comparing Fig. 9.6 to Fig. 9.1. Increasing Reynolds number results in decreased dissipation to nondimensional heat. There is, of course, an increase in dissipation to actual heat, but less rapidly than in the homogeneous case in which the dissipation rate increases as the square of the Reynolds number. Figure 9.6 shows that the dimensionless internal energy for the nonhomogeneous case $B$ is lower than that of the homogeneous case B. 


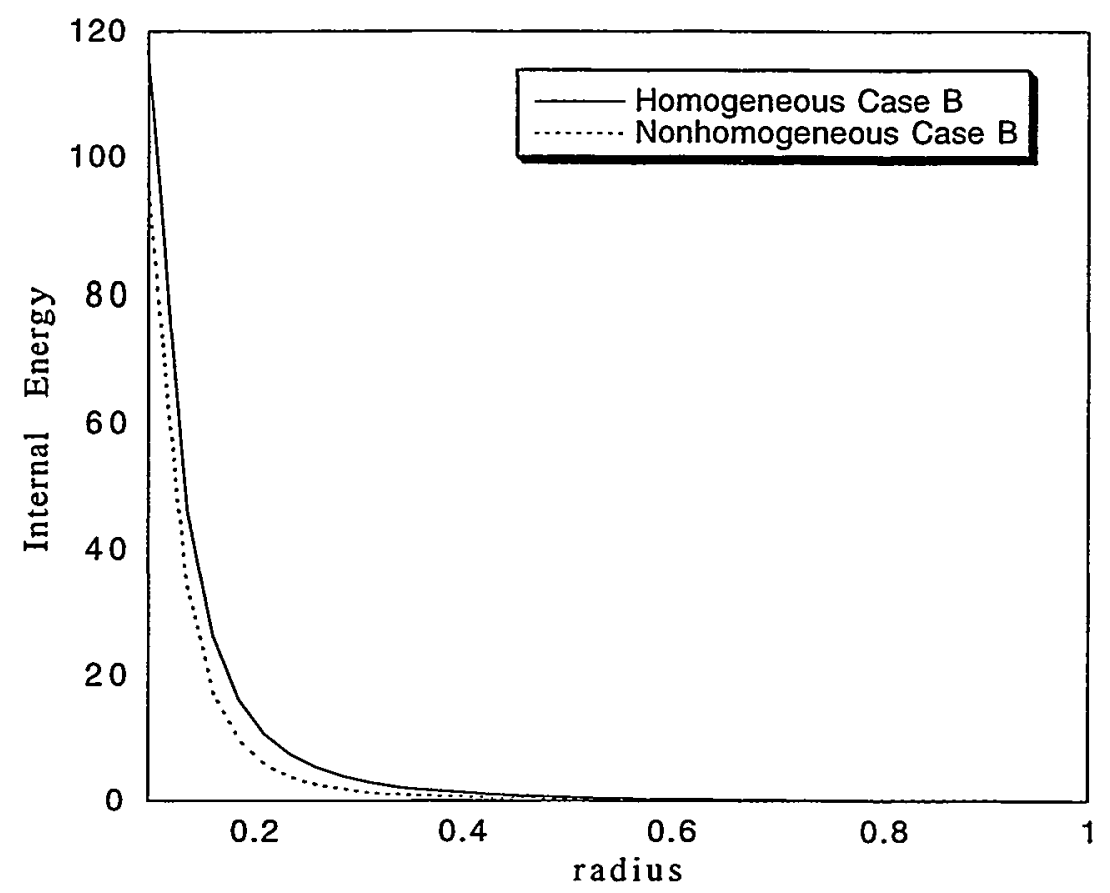

Figure 9.6: Comparison of Homogeneous Case B to Nonhomogeneous Case B

In the homogeneous version of case $\mathrm{C}$ the dimensionless internal energy is less than that of the homogeneous case with $\operatorname{Re}=100$, as seen by comparing Fig. 9.7 to Fig. 9.1. The dimensionless internal energy for the nonhomogeneous case $\mathrm{C}$ is lower than that of the homogeneous case $\mathrm{C}$, as seen in Fig. 9.7. 


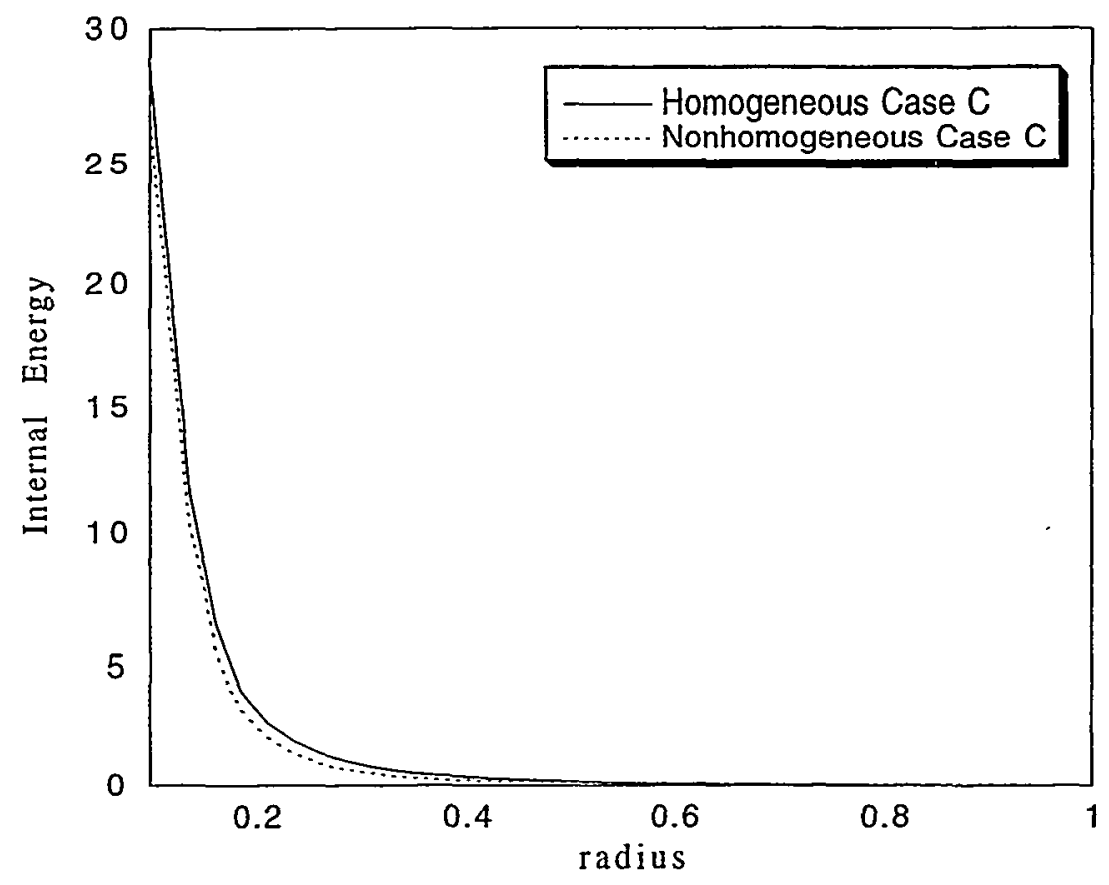

Figure 9.7: Comparison of Homogeneous Case $\mathrm{C}$ to Nonhomogeneous Case $\mathrm{C}$

As seen in the base case calculation, Fig. 9.2, the internal energy drops when there is some degree of nonhomogeneity in the system, no matter what the Reynolds number is.

When compared to the base case, the dimensionless turbulence energy has increased in case B and has decreased in case $\mathrm{C}$ as seen in Fig. 9.8. The actual (dimensionful) turbulence energy, however, appears from the calculations to be roughly independent of Reynolds number, in contrast to the behavior of turbulence energy in classical turbulence. This seeming paradox is related to the destructive (of Reynolds stress) coupling between $R_{i j}$ and the strain rate in spherical convergence, in contrast to the constructive coupling in shear flows. Thus the near constancy of $R_{i j}$ may result from a near balance of the destructive mean-flow coupling with the constructive coupling to $M_{i j}$. This is an open question that merits further investigation. 


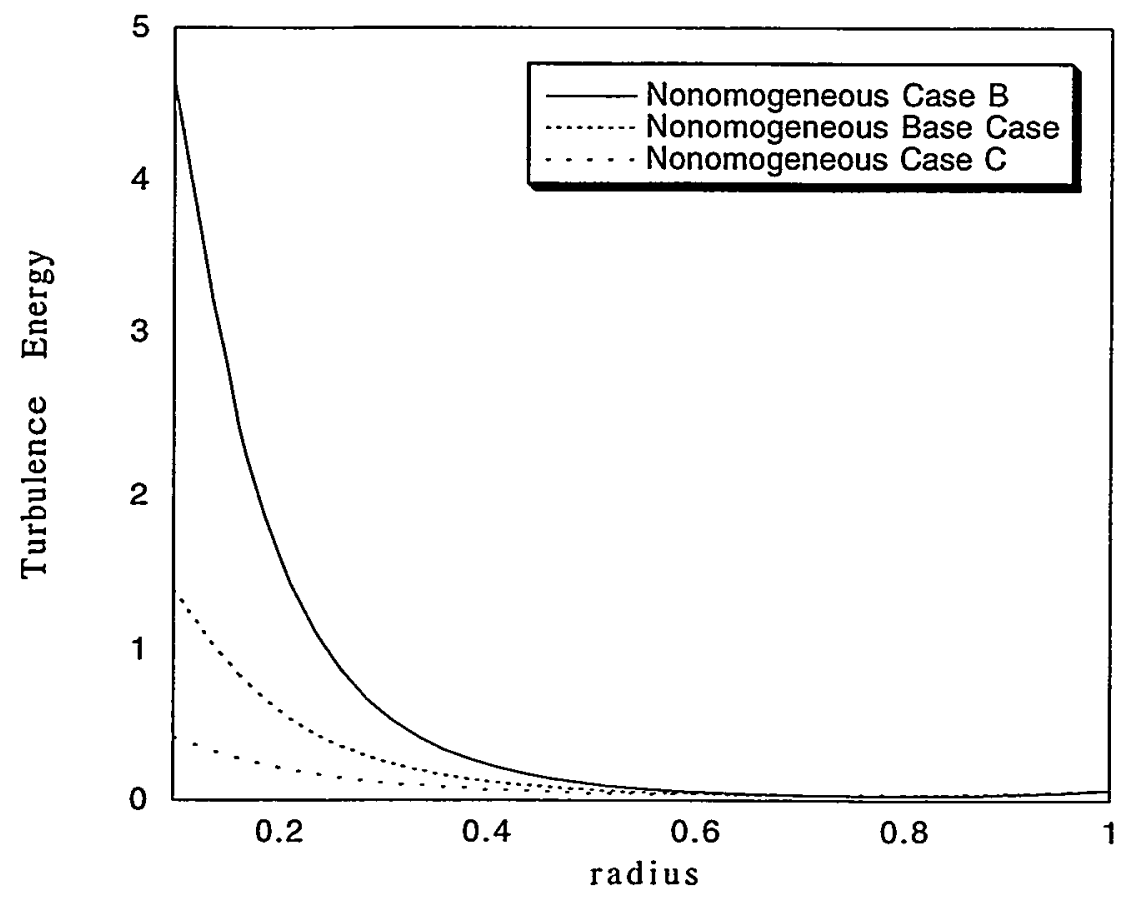

Figure 9.8: Turbulence Energy for the Base Case, Case B, and Case C

\subsection{Variation of $\beta$}

Variations of the nondimensional scale of nonhomogeneity $\left(\beta=\frac{s}{R_{2}}\right)$ have relatively little effect on the internal energy. In case $\mathrm{D}$ we decrease the value of $\beta$ by a factor of five from its value in the base case

Case D: $\operatorname{Re}=100 \quad \beta=0.01 \quad \gamma=0.5 \quad \eta=-1 \quad \hat{Z}_{(j j)}=100$

With this we observe that the internal energy is slightly larger than that for the nonhomogeneous base case, as shown in Fig. 9.9. 


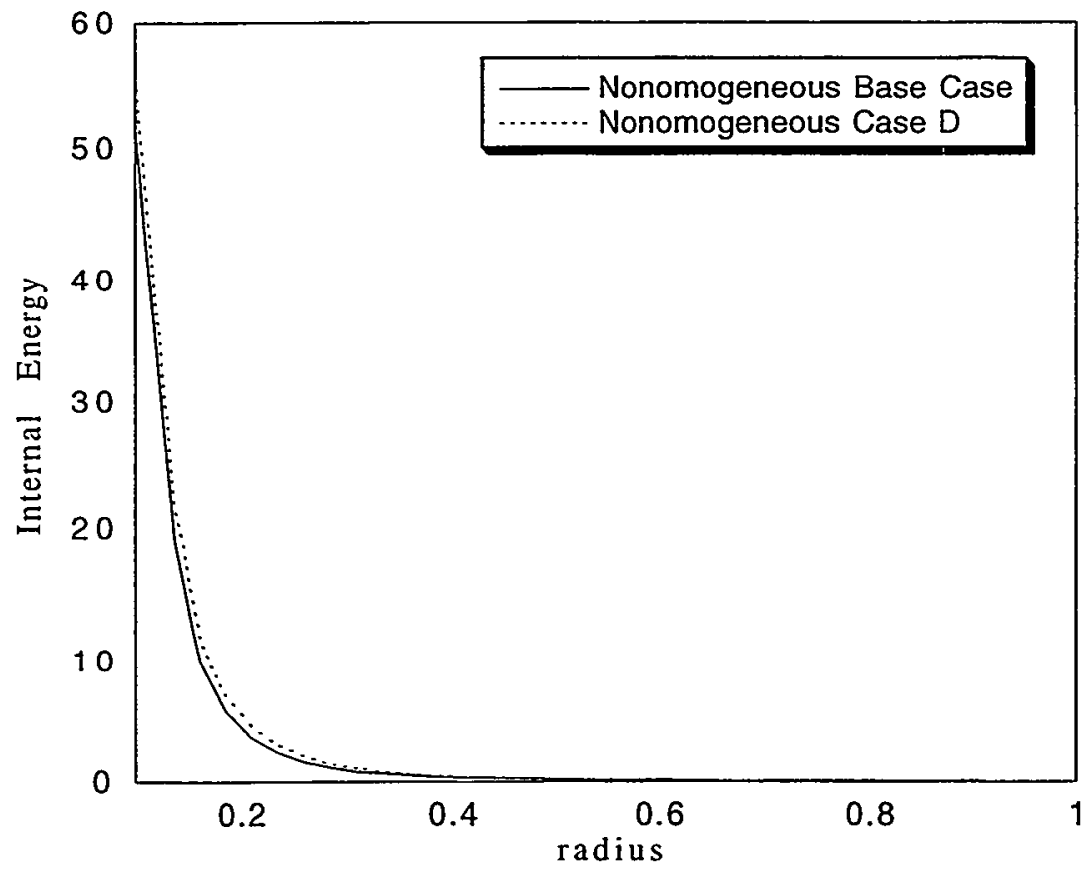

Figure 9.9: Comparison of Internal Energy for Nonhomogeneous Base Case and Nonhomogeneous Case D

The turbulence energy mostly decreases as $\beta$ decreases, as can be seen in Fig. 9.10. This result is not surprising, because we expect the turbulence energy to vanish as the system approaches a homogeneous state. 


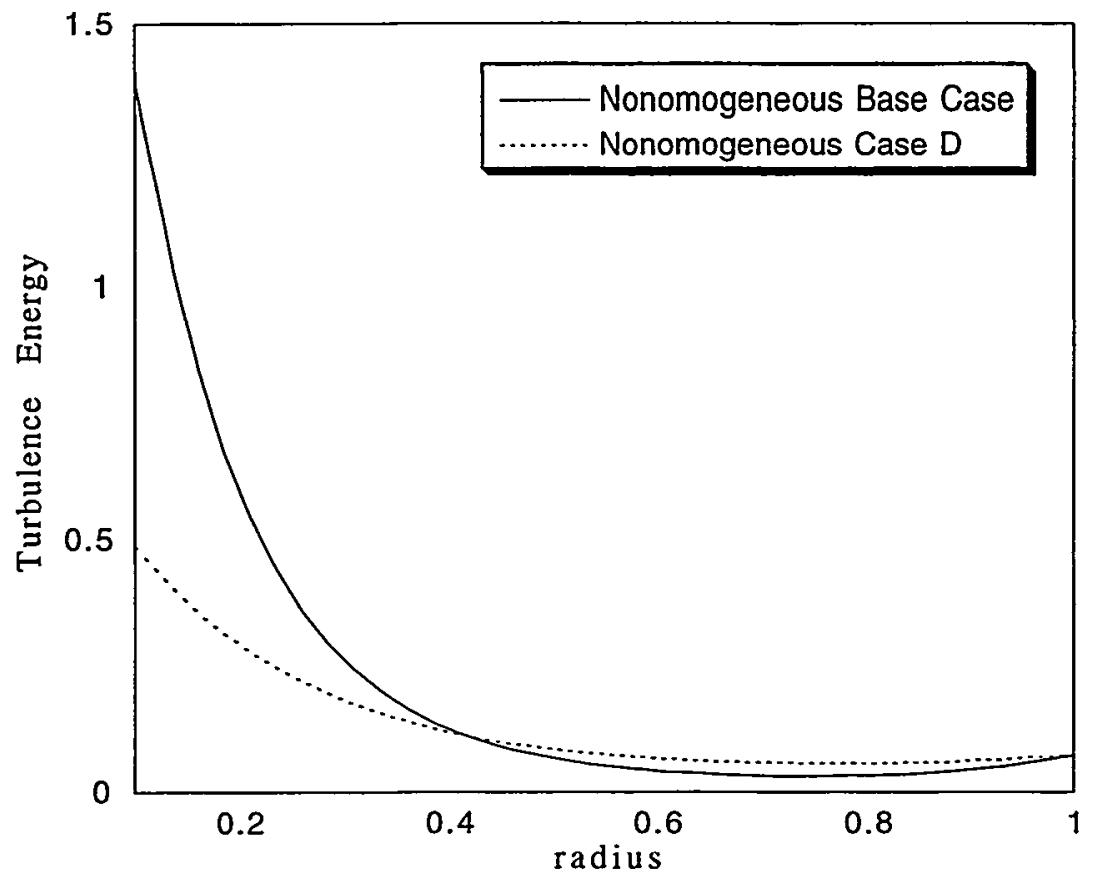

Figure 9.10: Comparison of Turbulence Energy for Nonhomogeneous Base Case and Nonhomogeneous Case D

The slight dip in the turbulence energy, for both the nonhomogeneous base case and the nonhomogeneous case $\mathrm{D}$, near the outer boundary is a consequence of the boundary condition of $\hat{K}=0.1$, at the inlet boundary.

\subsection{Variation of $\gamma$}

Decreasing the nondimensional fluctuations of viscosity $\left(\gamma=\frac{\delta v}{\bar{v}}\right)$ increases the internal energy towards that of the completely homogeneous case. In case $\mathrm{E}$ we decreased the value of $\gamma$ by sixty percent from the base case value

$$
\text { Case } \mathrm{E}: \operatorname{Re}=100 \quad \beta=0.05 \quad \gamma=0.2 \quad \hat{Z}_{(j j)}=100
$$


With this change, the dimensionless internal energy is larger than that for the nonhomogeneous base case, approaching the completely homogeneous case, as seen in Fig. 9.11

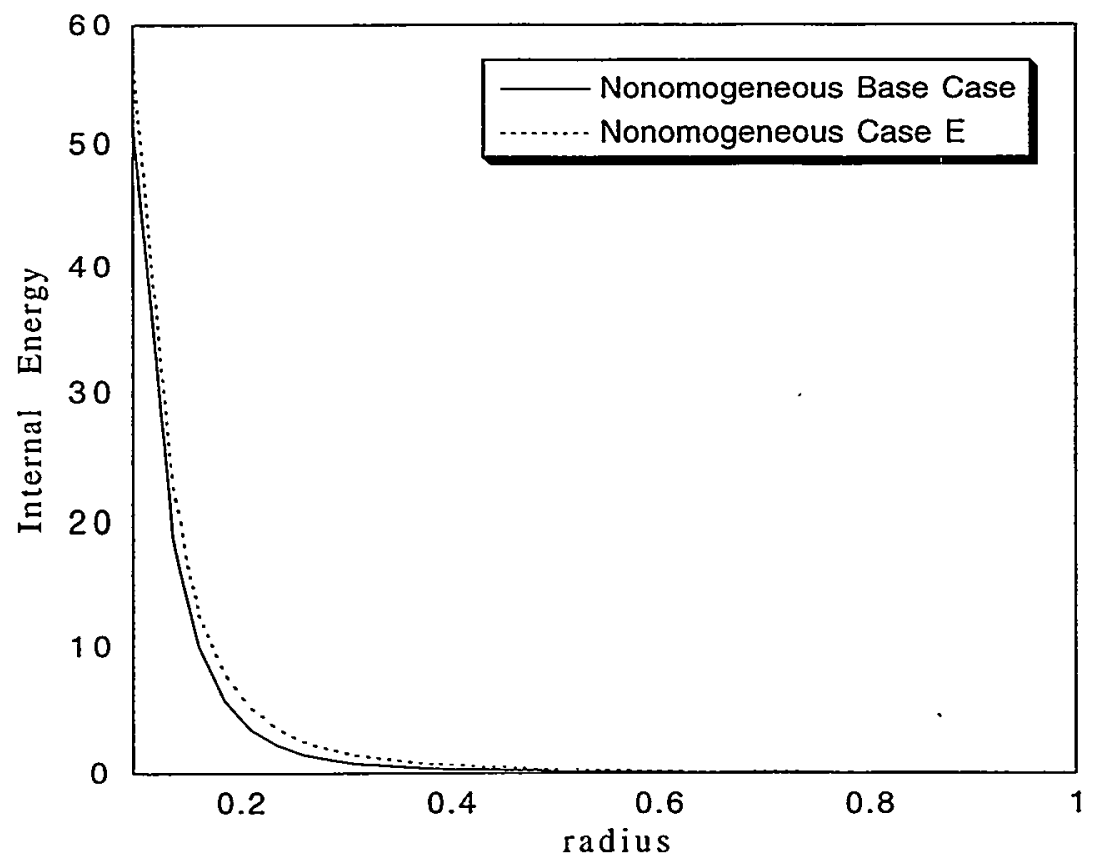

Figure 9.11: Comparison of Internal Energy for Nonhomogeneous Base Case and Nonhomogeneous Case E

The nondimensional turbulence energy decreases as $\gamma$ decreases, as can be seen in Fig. 9.12. This result is not surprising, because we would expect the turbulence energy to vanish as the system approaches a homogeneous state. 


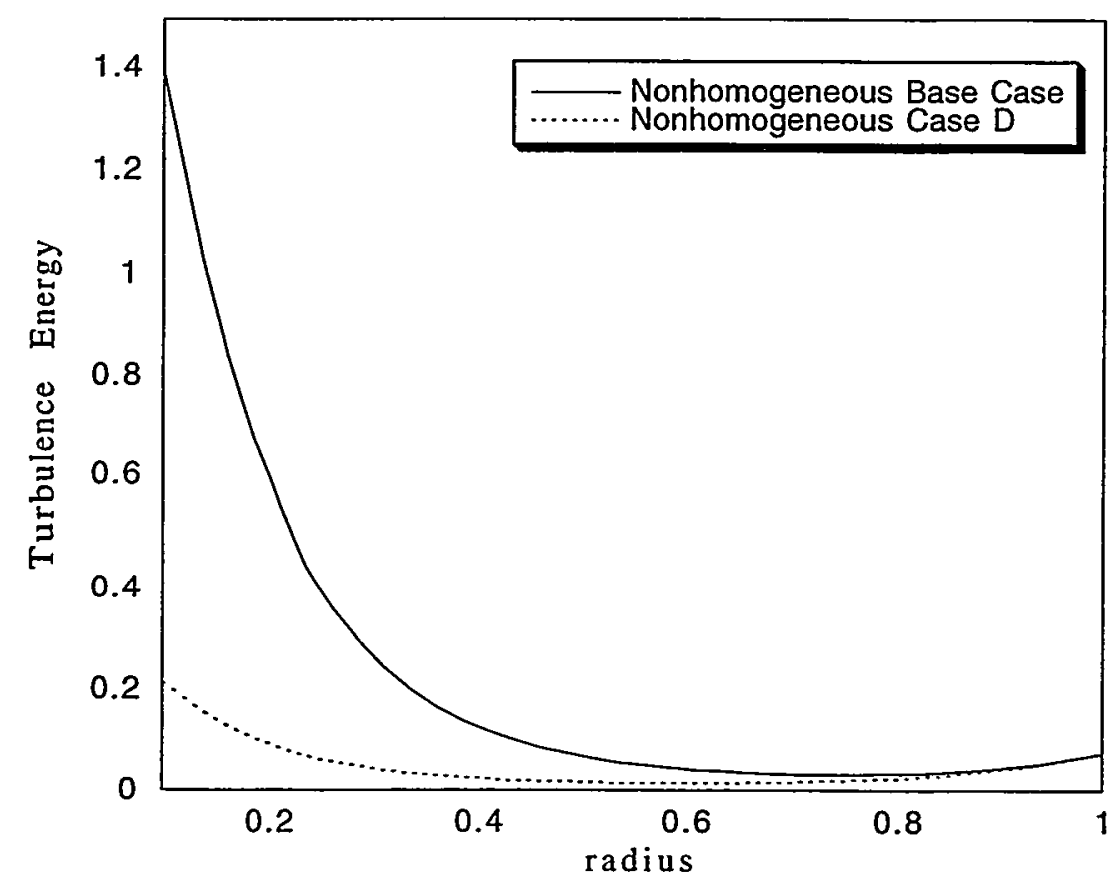

Figure 9.12: Comparison of Turbulence Energy for Nonhomogeneous Base Case and Nonhomogeneous Case E

The slight dip in the turbulence energy, for both the nonhomogeneous base case and the nonhomogeneous case $\mathrm{D}$ is a consequence of the boundary condition, $\hat{K}=0.1$, at the inlet boundary.

\subsection{Variations of $\hat{Z}_{(j j)}$}

The initial and boundary value of $\hat{Z}_{(j)}$ describes the strength of nonhomogeneity. Variations of $\hat{Z}_{(j j)}$ from the base-case value has an effect on all aspects of the results, including the internal energy and the turbulence energy. In case $F$ we decrease the level of incoming nonhomogeneity by decreasing $\hat{Z}_{(j i)}$. In case $\mathrm{G}$ we increase the level of incoming nonhomogeneity by increasing $\hat{Z}_{(j j)}$. 


$$
\text { Case F: } \operatorname{Re}=100 \quad \beta=0.05 \quad \gamma=0.5 \quad \hat{Z}_{(j j)}=50
$$

$$
\text { Case G: } \operatorname{Re}=100 \quad \beta=0.05 \quad \gamma=0.5 \quad \hat{Z}_{(j i)}=200
$$

Note that case $\mathrm{G}$ differs from case $\mathrm{A}$ only in having a smaller value of $\gamma$. With these changes from the base case we see in Fig. 9.13 a drop in the nondimensional internal energy for case $\mathrm{G}$ and a rise in that quantity for case $\mathrm{F}$.

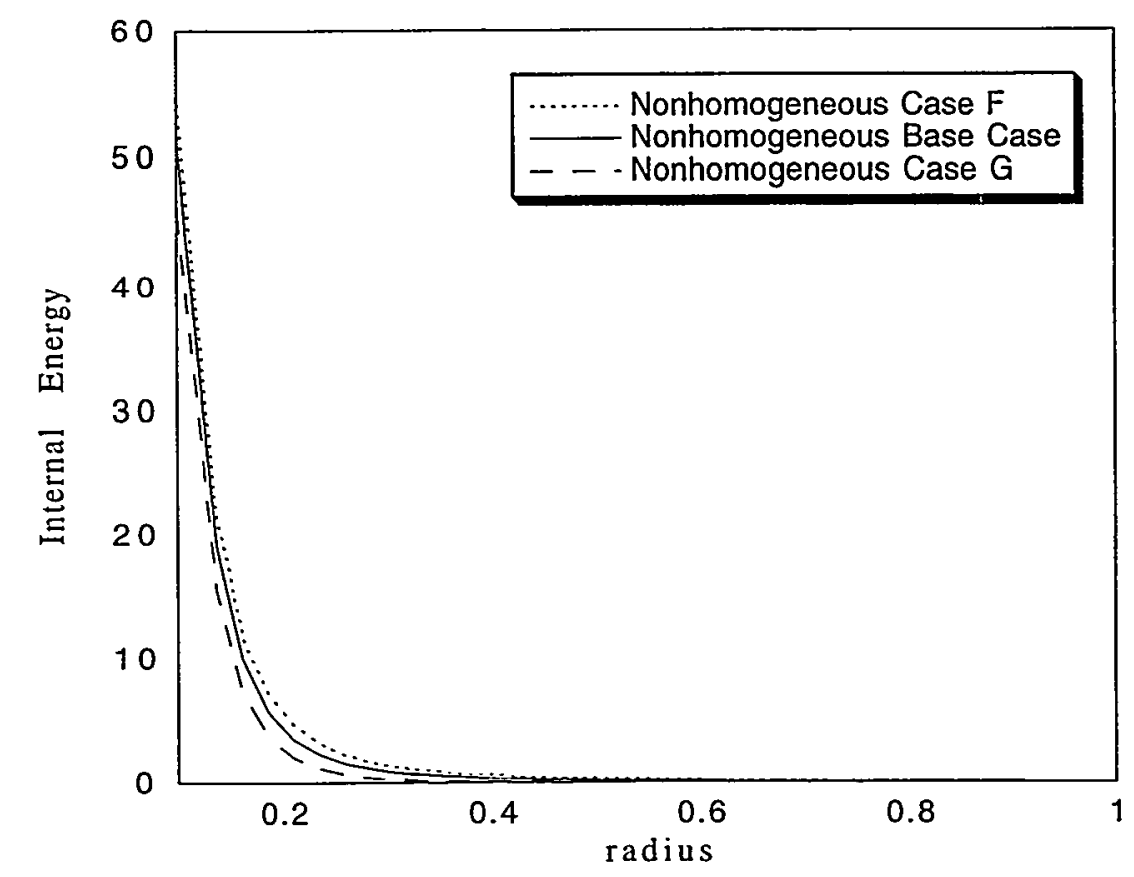

Figure 9.13: Comparison of Internal Energy for Nonhomogeneous Base Case, Nonhomogeneous Case F, and Nonhomogeneous Case G

This result is not surprising because we expect the internal energy to approach the internal energy for the homogeneous case as the level of nonhomogeneity vanishes. The turbulence energy increases for increasing $\hat{Z}_{(j j)}$ and decreases for decreasing $\hat{Z}_{(j))}$, as can be seen in Fig. 9.14. 


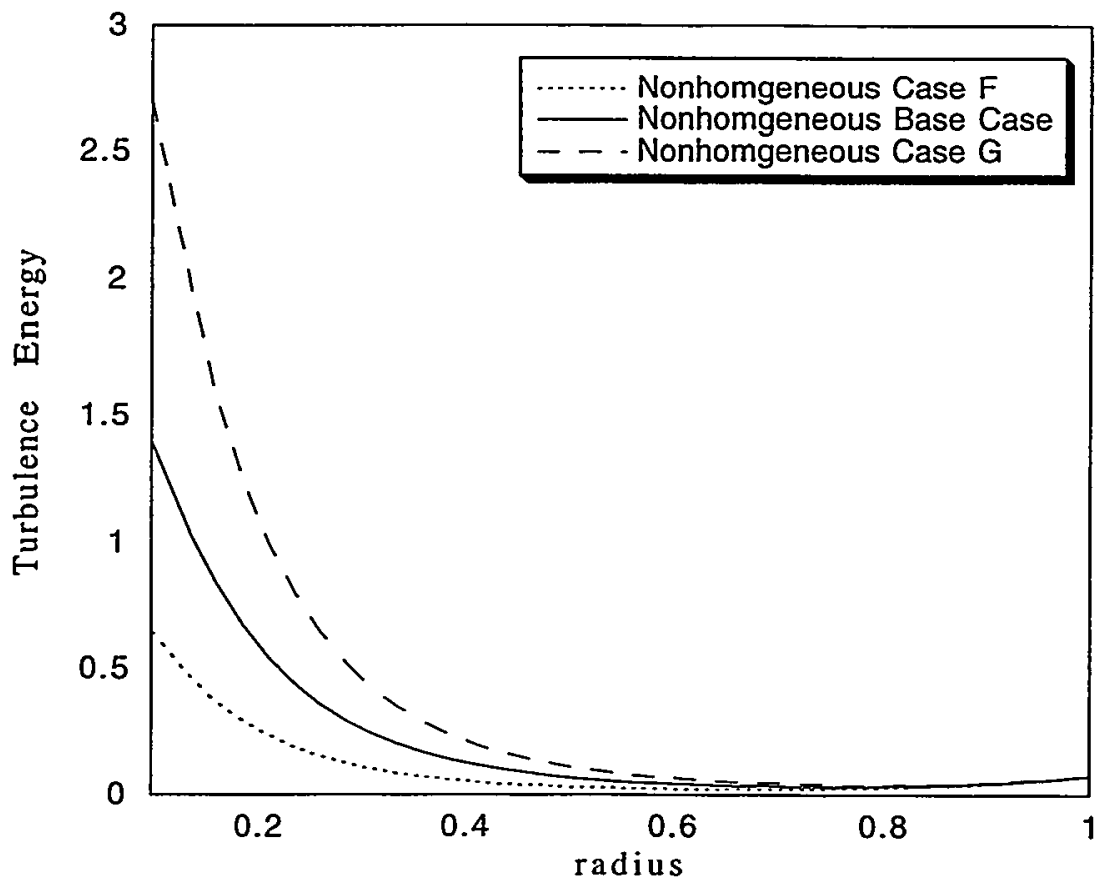

Figure 9.14: Comparison of Turbulence Energy for Nonhomogeneous Base Case, Nonhomogeneous Case F, and Nonhomogeneous Case G

\subsection{Enhancement to the Return to Isotropy}

As an example of varying one of the constant modeling parameters, we vary the $C_{H R}$ parameter in the term that describes return to isotropy in the $\hat{Z}_{i j}$ transport equation. We increase $C_{H R}$ from a value of 1.0 in the base case to a value of 5.0 in case $\mathrm{H}$.

Case H: $\operatorname{Re}=100 \quad \beta=0.05 \quad \gamma=0.5 \quad \hat{Z}_{(j)}=100 \quad C_{H R}=5.0$

By increasing $C_{H R}$ from its value in the base case, the nonhomogeneities do not elongate as much as the nonhomogeneities in the base case. The components of $\hat{Z}_{i j}$ are closer together than in the base case; this implies a lower level of anisotropy among the nonhomogeneities. The results can be seen in Fig. 9.15. 


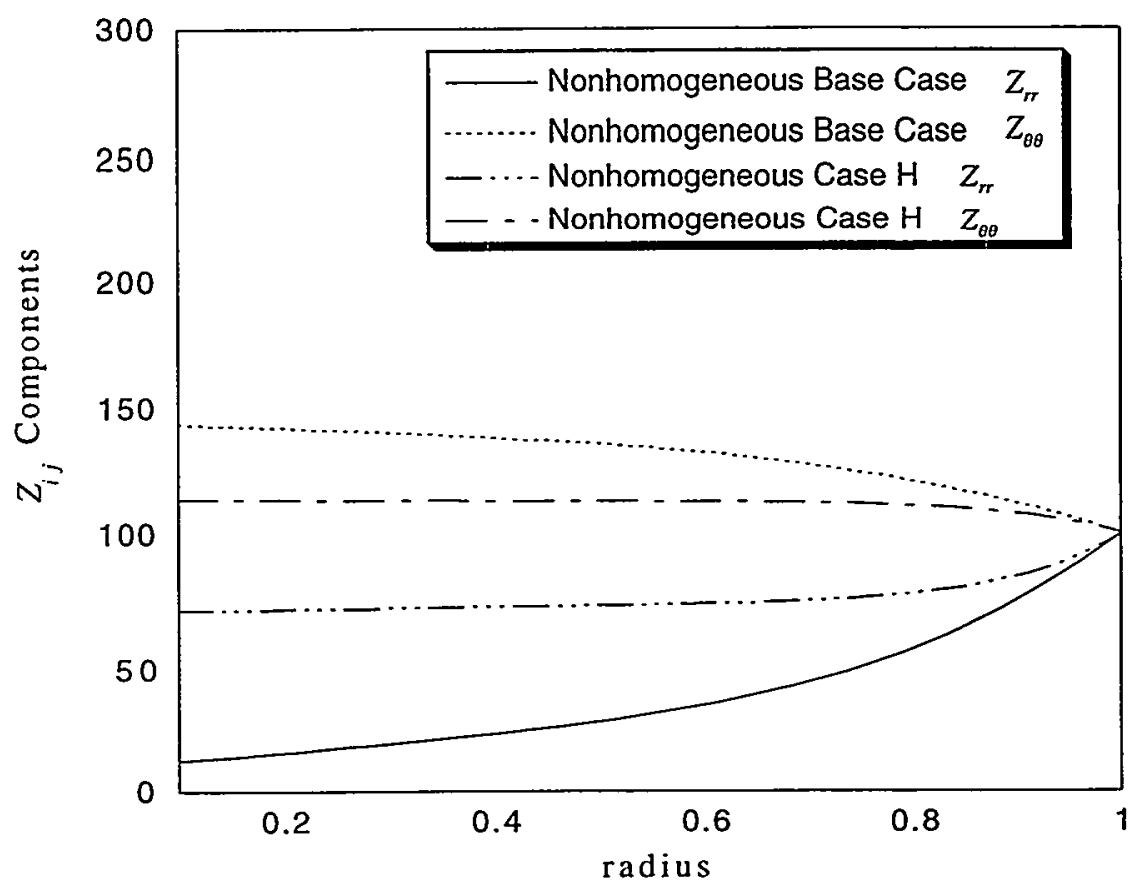

Figure 9.15: Comparison of $\hat{Z}_{i j}$ Component Distribution for the Nonhomogeneous

Base Case and Nonhomogeneous Case $\mathrm{H}$

Increasing $C_{H R}$ even more brings the $\hat{Z}_{i j}$ components even closer together, approaching a constant value equal to the boundary value. In general the value of $C_{H R}$ may not be constant at all, but a function of such quantities as the material strength. 


\subsection{Summary and Conclusions}

Crenulation is an instability usually observed in a converging fluid with nonhomogeneous stress response to strain and/or strain rate. In this thesis we have chosen the converging system to be a spherical shell of fluid in an Eulerian frame of reference, and the nonhomogeneity to be introduced through variations in the instantaneous kinematic viscosity. In general the nonhomogeneity could be introduced through variations in some other material property, or through surface imperfections.

We start with the Navier-Stokes equations for an incompressible Newtonian fluid. We make a Reynolds decomposition of the instantaneous velocity field, instantaneous pressure, and instantaneous kinematic viscosity while leaving the instantaneous mass density constant. The Reynolds decomposition yields a mean momentum equation, which receives contributions from the Reynolds stress $R_{i j}$ and from the first order response $M_{i j}$. This leads to the development of a transport equation for $R_{i j}$ and for $M_{i j}$. The transport equation for Reynolds stress contains a coupling of $M_{i j}$ to the rate-of-strain tensor. The transport equation for $M_{i j}$ in turn contains a coupling of $Z_{i j}$ to the rate-of-strain tensor. We also develop a transport equation for the driving tensor $Z_{i j}$, which describes the configuration of the nonhomogeneities in the system. Double and triple correlations arise in the transport-equation derivations. Some of the double and triple correlations are modeled using techniques from classical turbulence-transport modeling theories. Others also arise in the analysis requiring some new derivations or postulates for closure. Consistency forces us to model the double and triple correlations in the internal energy equation using the same methods as for the $R_{i j}$ transport equation, which is necessary for a proper balance between turbulence energy and internal energy.

To illustrate the crenulative process, we transform the equations to a spherical coordinate system. We investigate the primary driver of the system, the limit as the nonhomogeneities vanished, a first order approximation for $Z_{i j}$, the effects of uniform and nonuniform distributions of instantaneous kinematic viscosity, and the constraints for 
positive-definite entropy changes in the fluid. The primary driver is $Z_{i j}$, which couples to the rate-of-strain tensor in the $M_{i j}$ equation. In the absence of initial $R_{i j}$ and $M_{i j}, Z_{i j}$ develops the first-order response to crenulation $\left(M_{i j}\right)$, then a second order response $\left(R_{i j}\right)$.

In the limit as the nonhomogeneities vanish a classical turbulence model emerges from the crenulation turbulence model. The separation scale, $s$, between nonhomogeneities becomes instead the dominant scale of the turbulence itself.

Nondimensionalizing the governing equations and boundary conditions results in four nondimensional parameters $\operatorname{Re}, \beta, \gamma$, and the initial value of $\hat{Z}_{(j j)}$. These parameters allow us to characterize crenulative turbulence much more efficiently than with the dimensionful parameters. A significant consequence of crenulation is manifested in the balance among the energies, in particular the relative values of internal energy and turbulence energy in comparison to the total mean-flow kinetic energy. The crenulative process always reduces the rate of dissipation to heat below that of the purely homogeneous case with the same mean viscosity, $\bar{v}$. This effect is especially apparent for configurations in which the low-viscosity regions are thin channels that allow the regions of high viscosity to slip easily past each other.

Crenulation occurs in any circumstance in which the mean-flow stream lines converge the material more strongly than the compressibility can accommodate. For a homogeneous material, crenulation occurs at the atomic or molecular scale. With nonhomogeneous stress response to strain and/or strain rate at larger scales, the crenulative process also occurs at those larger scales. The results are manifested by a decrease in the rate of dissipation to heat, and by the configurationally-irreversible mixing of nonhomogeneities across any mean-flow-transported interface. A closely related process also occurs in mean-flow shear, in which transport of the nonhomogeneities can result in mixing of materials across the slip surface. 
The next direction for our investigations is the application of crenulation theory to granular metals, for which the nonhomogeneities occur through variations in material yield strength, and in the moduli for elastic and plastic flow. 


\section{Appendix A}

\section{Numerics}

The numerical algorithm used to solve the set of governing equations is an explicit time-marching finite-difference technique in a one dimensional spherical configuration along the radius. The stochastically steady state solution is found by time marching. Because of the finite difference arrangement an imaginary control volume at both boundaries is needed to implement the boundary conditions. A convergence test was conducted and compared to the analytic solution,.

\section{A.1 Numerical Scheme}

The explicit approximation of the $r r$-component of the Reynolds stress equation is

$$
\begin{gathered}
\hat{R}_{r r}^{n+1}=\hat{R}_{r r}^{n}+\Delta t\left\{4 \eta \frac{\hat{R}_{r r}^{n}}{\hat{r}^{3}}-\eta\left(\frac{\partial}{\partial \hat{r}}\left(\frac{\hat{R}_{r r}^{n}}{\hat{r}^{2}}\right)+\frac{2 \hat{R}_{r r}^{n}}{\hat{r}^{3}}\right)+\frac{1}{\hat{r}^{2}} \frac{\partial}{\partial \hat{r}}\left[\hat{r}^{2} 2 C_{D} \beta \sqrt{\hat{K}^{n}} \frac{\partial \hat{R}_{r r}^{n}}{\partial \hat{r}}\right]\right. \\
-4 C_{D} \beta \sqrt{\hat{K}^{n}} \frac{\left(2 \hat{R}_{r r}^{n}-\hat{R}_{\theta \theta}^{n}-\hat{R}_{\phi \phi}^{n}\right)}{\hat{r}^{2}}+C_{B} \eta\left[-4 \frac{\hat{R}_{r r}^{n}}{\hat{r}^{3}}-\frac{2}{3}\left(-2 \frac{\hat{R}_{r r}^{n}}{\hat{r}^{3}}+\frac{\hat{R}_{\theta \theta}^{n}}{\hat{r}^{3}}+\frac{\hat{R}_{\phi \phi}^{n}}{\hat{r}^{3}}\right)\right] \\
+\left(\frac{C_{R} \sqrt{\hat{K}^{n}}}{\beta}+\frac{C_{H R} \sqrt{6}}{\hat{r}^{3}}\right)\left(\frac{2}{3} \hat{K}^{n}-\hat{R}_{r r}^{n}\right)-8 \eta\left(\frac{\gamma}{\operatorname{Re} \beta}\right)^{2} \frac{\hat{M}_{r r}^{n}}{\hat{r}^{3}} \\
\left.+\frac{1}{\operatorname{Re}}\left[\frac{1}{\hat{r}^{2}} \frac{\partial}{\partial \hat{r}}\left(\hat{r}^{2} \frac{\partial \hat{R}_{r r}^{n}}{\partial \hat{r}}\right)-2 \frac{\left(2 \hat{R}_{r r}^{n}-\hat{R}_{\theta \theta}^{n}-\hat{R}_{\phi \phi}^{n}\right)}{\hat{r}^{2}}\right]-\frac{1}{\operatorname{Re} \beta^{2}}(2+f) \Delta \hat{R}_{r r}^{n}\right\}
\end{gathered}
$$


The explicit approximation of the $r r$-component of the $M_{i j}$ transport equation is

$$
\begin{gathered}
\hat{M}_{r r}^{n+1}=\hat{M}_{r r}^{n}+\Delta t\left\{4 \eta \frac{\hat{M}_{r r}^{n}}{\hat{r}^{3}}+\frac{2}{3} \eta\left(-2 \frac{\hat{M}_{r r}^{n}}{\hat{r}^{3}}+\frac{\hat{M}_{\theta \theta}^{n}}{\hat{r}^{3}}+\frac{\hat{M}_{\phi \phi}^{n}}{\hat{r}^{3}}\right)-\eta\left(\frac{\partial}{\partial \hat{r}}\left(\frac{\hat{M}_{r r}^{n}}{\hat{r}^{2}}\right)+\frac{2 \hat{M}_{r r}^{n}}{\hat{r}^{3}}\right)\right. \\
+\frac{1}{\hat{r}^{2}} \frac{\partial}{\partial \hat{r}}\left[\hat{r}^{2} 2 C_{H} \beta \sqrt{\hat{K}^{n}} \frac{\partial \hat{M}_{r r}^{n}}{\partial \hat{r}}\right]-4 C_{H} \beta \sqrt{\hat{K}^{n}} \frac{\left(2 \hat{M}_{r r}^{n}-\hat{M}_{\theta \theta}^{n}-\hat{M}_{\phi \phi}^{n}\right)}{\hat{r}^{2}} \\
-\left[\frac{\left(2+f_{I}\right) \Delta}{\operatorname{Re} \beta^{2}}+\frac{C_{M} \sqrt{\hat{K}^{n}}}{\beta}+\frac{C_{H R} \sqrt{6}}{\hat{r}^{3}}\right] \hat{M}_{r r}^{n}-4 \eta \frac{\hat{Z}_{r r}^{n}}{\hat{r}^{3}}-\frac{2}{3} \eta\left(-2 \frac{\hat{Z}_{r r}^{n}}{\hat{r}^{3}}+\frac{\hat{Z}_{\theta \theta}^{n}}{\hat{r}^{3}}+\frac{\hat{Z}_{\phi \phi}^{n}}{\hat{r}^{3}}\right) \\
\left.+\frac{1}{\operatorname{Re}} \frac{1}{\hat{r}^{2}} \frac{\partial}{\partial \hat{r}}\left[\hat{r}^{2} \frac{\partial \hat{M}_{r r}^{n}}{\partial \hat{r}}\right]-\frac{2}{\operatorname{Re}} \frac{\left(2 \hat{M}_{r r}^{n}-\hat{M}_{\theta \theta}^{n}-\hat{M}_{\phi \phi}^{n}\right)}{\hat{r}^{2}}\right\}
\end{gathered}
$$

The explicit approximation of the $r r$-component of the $Z_{i j}$ transport equation is

$$
\begin{gathered}
\hat{Z}_{r r}^{n+1}=\hat{Z}_{r r}^{n}+\Delta t\left\{4 \eta \frac{\hat{Z}_{r r}^{n}}{\hat{r}^{3}}+\frac{2}{3} \eta\left(-2 \frac{\hat{Z}_{r r}^{n}}{\hat{r}^{3}}+\frac{\hat{Z}_{\theta \theta}^{n}}{\hat{r}^{3}}+\frac{\hat{Z}_{\phi \phi}^{n}}{\hat{r}^{3}}\right)-\eta\left(\frac{\partial}{\partial \hat{r}}\left(\frac{\hat{Z}_{r r}^{n}}{\hat{r}^{2}}\right)+\frac{2 \hat{Z}_{r r}^{n}}{\hat{r}^{3}}\right)\right. \\
{\left[\frac{C_{Z} \sqrt{\hat{K}^{n}}}{\beta}+\frac{C_{H R} \sqrt{6}}{\hat{r}^{3}}\right]\left[\frac{1}{3}\left(\hat{Z}_{r r}^{n}+\hat{Z}_{\theta \theta}^{n}+\hat{Z}_{\phi \phi}^{n}\right)-\hat{Z}_{r r}^{n}\right]+\frac{1}{\hat{r}^{2}} \frac{\partial}{\partial \hat{r}}\left[\hat{r}^{2} 2 C_{X} \beta \sqrt{\hat{K}^{n}} \frac{\partial \hat{Z}_{r r}^{n}}{\partial \hat{r}}\right]} \\
\left.-4 C_{X} \beta \sqrt{\hat{K}^{n}} \frac{\left(2 \hat{Z}_{r r}^{n}-\hat{Z}_{\theta \theta}^{n}-\hat{Z}_{\phi \phi}^{n}\right)}{\hat{r}^{2}}-\frac{1}{3} \frac{1}{\hat{r}^{2}} \frac{\partial}{\partial \hat{r}}\left[\hat{r}^{2} 2 C_{X} \beta \sqrt{\hat{K}^{n}} \frac{\partial\left(\hat{Z}_{r r}^{n}+\hat{Z}_{\theta \theta}^{n}+\hat{Z}_{\phi \phi}^{n}\right)}{\partial \hat{r}}\right]\right\}
\end{gathered}
$$

The explicit approximation of the internal energy transport equation is

$$
\begin{gathered}
\hat{\bar{e}}^{n+1}=\hat{\bar{e}}^{n}+\Delta t\left\{-\eta\left(\frac{\partial}{\partial \hat{r}}\left(\frac{\hat{\bar{e}}^{n}}{\hat{r}^{2}}\right)+\frac{2 \hat{\bar{e}}^{n}}{\hat{r}^{3}}\right)+\frac{12}{\operatorname{Re} \hat{r}^{6}}+\frac{(2+f) \Delta \hat{K}^{n}}{\operatorname{Re} \beta^{2}}\right. \\
\left.+\frac{1}{\operatorname{Re} \hat{r}^{2}} \frac{\partial}{\partial \hat{r}}\left[\frac{\partial}{\partial \hat{r}}\left(\hat{r}^{2} \hat{R}_{r r}^{n}\right)-\hat{r}\left(\hat{R}_{\theta \theta}^{n}+\hat{R}_{\phi \phi}^{n}\right)\right]-4 \eta\left(\frac{\gamma}{\operatorname{Re} \beta}\right)^{2}\left(-2 \frac{\hat{M}_{r r}^{n}}{\hat{r}^{3}}+\frac{\hat{M}_{\theta \theta}^{n}}{\hat{r}^{3}}+\frac{\hat{M}_{\phi \phi}^{n}}{\hat{r}^{3}}\right)\right\}
\end{gathered}
$$

Equations for the other components of the tensors are written in similar fashion. The superscripts $n$ and $n+1$ indicate values at time $n \Delta t$ and time $(n+1) \Delta t$, respectively. 
Under the explicit time marching scheme, in which the mean velocity field is held constant in time, the Reynolds stress, $M_{i j}$, and $Z_{i j}$ components at time $n+1$ are solved as functions of the variables at time $n$. The variables are spatially discretized on a collocated grid, in which the values of the variables are located at the centers of control volumes, except for the mean velocity as in Fig. 8.1. The mean velocity can be located at the center or at the edges of a control volume, since it is a continuous function of position only. The grid spacing is uniform in the radial direction.

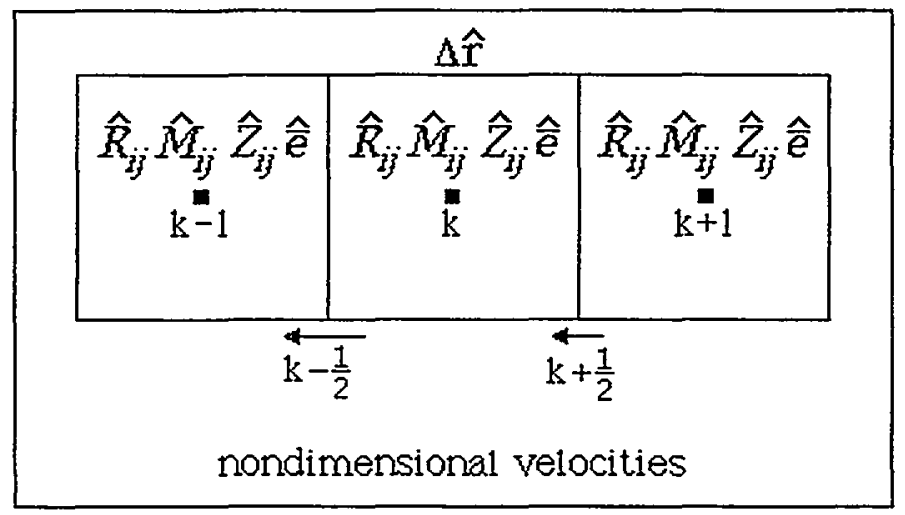

Figure A.1: Finite Difference Grid

A second-order upwind difference scheme is used for the discretization of the advection terms in each of the governing equations. The upwind scheme is different if the spherical system is converging or diverging.

For our examples, $\eta<0$ so that for some variable $L$

$$
\left[\frac{1}{\hat{r}^{2}} \frac{\partial L}{\partial \hat{r}}\right]_{k} \approx \eta\left[\frac{\left(L_{k+1}-L_{k}\right)}{\hat{r}_{k+\frac{1}{2}}^{2} \Delta \hat{r}}\right]
$$




\section{A.2 Boundary Conditions in Finite Difference Form}

An imaginary control volume is needed at each end of the finite difference domain as seen in Fig. A.2.

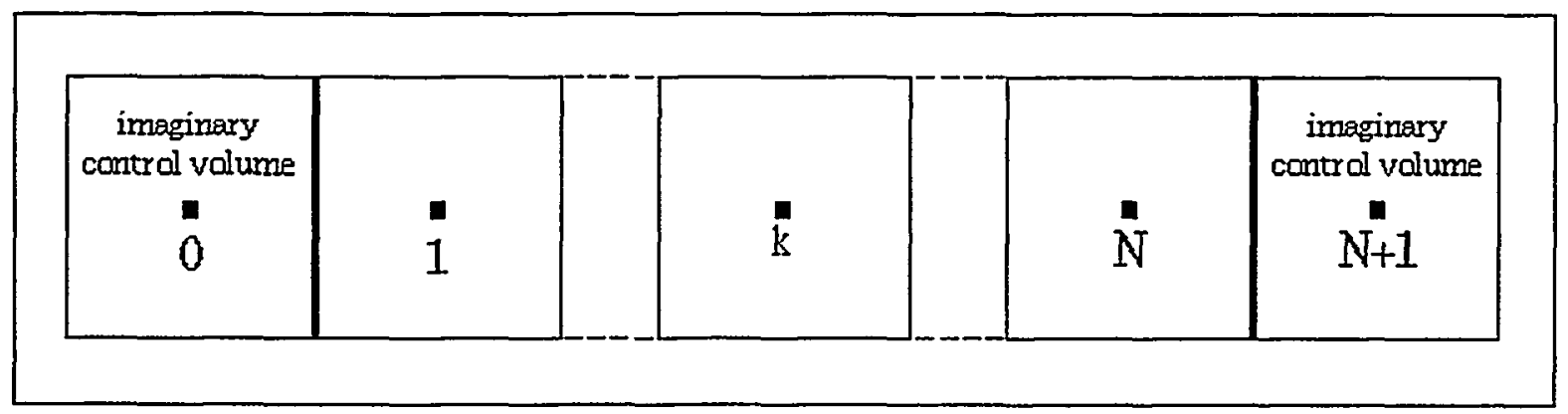

Figure A.2: Finite Difference Domain with Imaginary Control Volumes

These imaginary control volumes are needed to implement the boundary conditions. The values of the variables in control volume number 0 is obtained by a linear extrapolation from control volumes 1 and 2

$$
L_{o}=\frac{\left(r_{o}-r_{2}\right)}{\left(r_{2}-r_{1}\right)}\left(L_{2}-L_{1}\right)+L_{2}
$$

The values of the variables in control volume number $\mathrm{N}+1$, where $\mathrm{N}$ is the last control volume in the domain, are calculated knowing the prescribed value at the boundary and the value just inside the boundary

$$
L_{N+1}=2 L_{\text {Boundary }}-L_{N}
$$




\section{A.3 Convergence Test for Homogeneous Case}

A numerical convergence test of the homogeneous case was conducted and compared to the analytic solution. The first calculation was done with $\Delta \hat{r}=0.025$ and the solution displayed good qualitative results. The second calculation was done with $\Delta \hat{r}=0.005$ and the solution is quite close to the exact (analytic) solution, as can be seen in Fig. A.3.

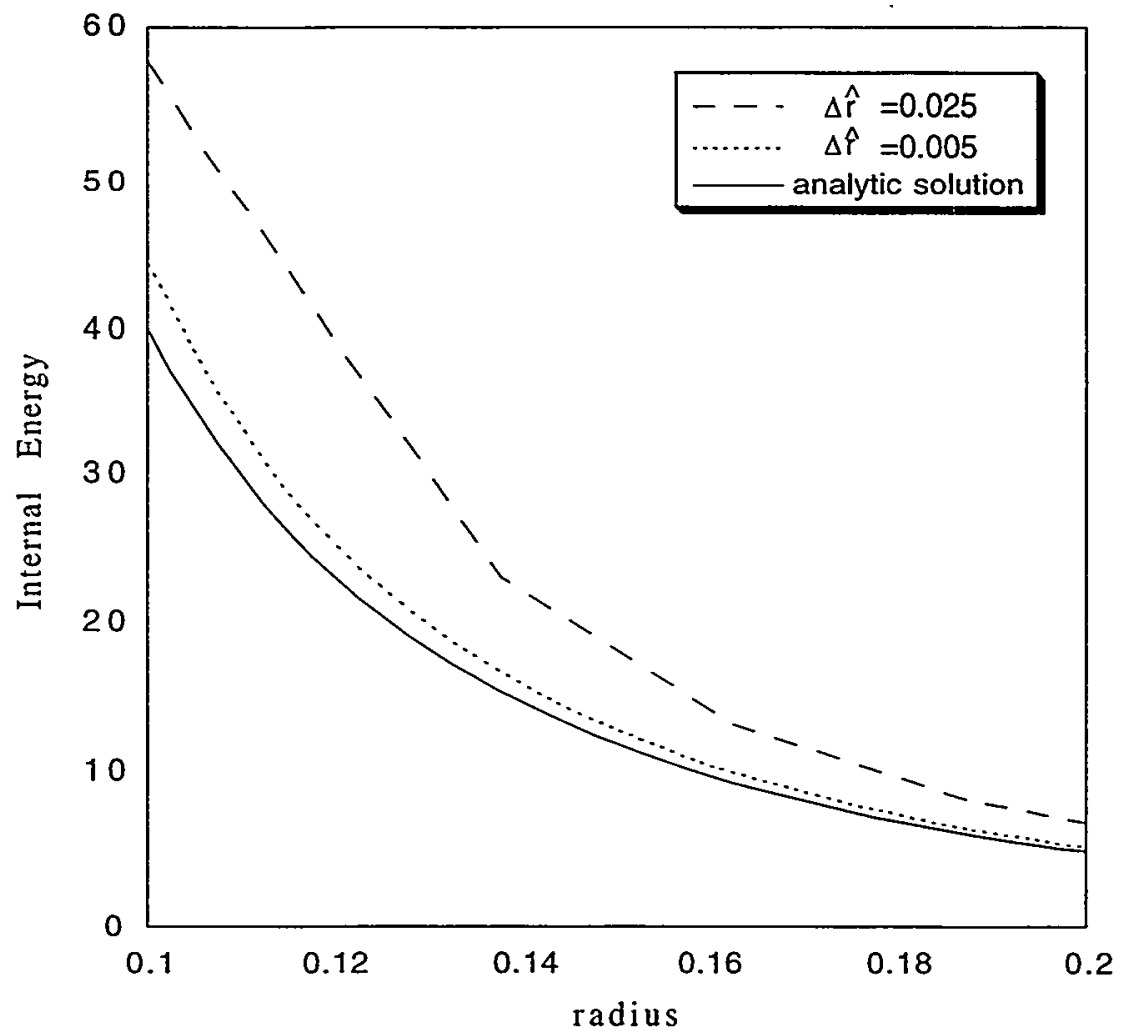

Figure A.3: Convergence Test for Homogeneous Case

The calculation with a resolution of $\Delta \hat{r}=0.025$ took about six times less time than the calculation with a resolution of $\Delta \hat{r}=0.005$. To display the trends due to crenulation it is sufficient to do these calculations with $\Delta \hat{r}=0.025$. 


\section{References}

[1] F.H. Harlow and A.A. Amsden. Fluid Dynamics. Technical Report LA-4700, Los Alamos Scientific Laboratory, Los Alamos, NM (1971).

[2] H. Lamb, Hydrodynamics, 6th Ed., Dover Publications, New York

[3] G.I. Bell. "Taylor Instability on Cylinders and Spheres in the Small Amplitude Approximations". Technical Report LA-1321, Los Alamos Scientific Laboratory, Los Alamos, NM (1951).

[4] M.S. Plesset. "On the Stability of Fluid Flows with Spherical Symmetry". Journal of Applied Physics, 25 (1954).

[5] W.W. Hsing, C.W. Barnes, J.B. Beck, and N.M. Hoffman. "Rayleigh-Taylor instability evolution in ablatively driven cylindrical implosions". Paper 7IA1, Bull. American. Physical. Society. 41, 1529 (1996).

[6] W.W. Hsing and N.M. Hoffman. "Measurement of Feedthrough and Instabililty Growth in Radiation-Driven Cylindrical Implosions". Paper 7IA1, Bull. American. Physical. Society. 41, 1529 (1997).

[7] P.V. Makarov. "Microdynamic Theory of Plasticity and Failure of Structurally Inhomogeneous Media". Russian Physics Journal, Vol. 35, No. 4. April 1992

[8] Brad Holian and Peter Lomdahl, Los Alamos National Laboratory, personal communication, June 1997.

[9] B. Trent. "The Effect of Micro-Structure on the Macroscopic Behavior of Cemented Granular Material". Ph.D. Thesis in Geo-Engineering at the University of Minnesota, (1998); Technical Report LA-11169, Los Alamos Scientific Laboratory, Los Alamos, NM (1988).

[10] B.D. Daly and F.H. Harlow. "Transport Equations in Turbulence". Physics of Fluids 13, 2634, (1970).

[11] R.R. Linn. "Effects of Modeled Terms in the Reynolds-Stress Transport Equations". Master of Science Thesis in Mechanical Engineering at the University of Illinois at Urbana-Champaign, (1993).

[12] B.E. Launder, G. J. Reece, and W. Rodi. "Progress in the Development of a Reynolds-stress Turbulence Closure". Journal of Fluid Mechanics 68:537-566 (1975).

[13] J. Rotta, "Statistische Theorie Nichthomogener Turbulenz". Z. Physik (1951)

[14] D. Bernard, F.H. Harlow, R.M. Rauenzahn, and C. Zemach. "Turbulence Transport Equations for Variable-Density Turbulence and Their Relationship to Two-Field Models". Los Alamos National Laboratory Report No. LA-12303-MS, (1992). 
[15] I.G. Currie. Fundamental Meachanics of Fluids. McGraw-Hill, Inc., New York, NY, second edition, (1993).

[16] R.B. Bird, R.C. Armstrong, and O. Hassanger. Dynamics of Polymeric Liquids. John Wiley \& Sons, Inc., New York, NY, volume 1, (1977).

[17] M. Shahinpoor. "Advances in the Mechanics and the Flow of Granular Materials". Trans Tech Publications, Clausthal, Germany, volume 2, (1983).

[18] H.N. Fisher. "Instability in Converging Compressible Systems". Los Alamos National Laboratory memorandum X-1(5/82)22 (September 1982). 